\title{
ANALYSIS AND APPLICATIONS: THE MATHEMATICAL WORK OF ELIAS STEIN
}

\author{
CHARLES FEFFERMAN, ALEX IONESCU, TERENCE TAO, AND STEPHEN WAINGER, \\ WITH CONTRIBUTIONS FROM LOREDANA LANZANI, AKOS MAGYAR, \\ MARIUSZ MIREK, ALEXANDER NAGEL, D. H. PHONG, LILLIAN PIERCE, \\ FULVIO RICCI, CHRISTOPHER SOGGE, AND BRIAN STREET
}

Abstract. This article discusses some of Elias M. Stein's seminal contributions to analysis.

\section{Contents}

Part I. Selections from Stein's classical results

I.1. Complex interpolation

I.2. Curvature and the Fourier transform

I.3. $H^{p}$-spaces 528

I.4. The Cotlar-Stein lemma 532

I.5. Representation theory 534

I.6. $\bar{\partial}$-problems 538

I.7. Conclusions

Part II. Recent advances and future directions 547

II.1. Continuous and discrete Radon transforms 547

II.2. Pseudoconvexity and the Cauchy-Szegő projection

II.3. Dimension-free estimates and variation norms

II.4. Multiparameter singular integrals and applications 5

II.5. Singular Radon transforms and the $\bar{\partial}$-Neumann problem

II.6. Polynomial Carleson operators $\quad 569$

II.7. Oscillatory integrals and the role of curvature $\quad 572$

II.8. Multiparameter singular Radon transforms

II.9. The restriction conjecture 576

Part III. Appendix: Eli Stein's Bibliography 578

References

Received by the editors December 13, 2019.

2010 Mathematics Subject Classification. Primary 32-02, 35-02, 42-02. 


\title{
Part I. Selections from Stein's classical results
}

\author{
contributed by C. Fefferman 1
}

The purpose of this survey article is to give the general reader some idea of the scope and originality of Eli Stein's contributions to analysis up to the year 1991. His work deals with representation theory, classical Fourier analysis, and partial differential equations. He was the first to appreciate the interplay among these subjects, and to perceive the fundamental insights in each field arising from that interplay. No one else really understands all three fields; therefore, no on else could have done the work I am about to describe. However, deep understanding of three fields of mathematics is by no means sufficient to lead to Stein's main ideas. Rather, at crucial points, Stein has shown extraordinary originality, without which no amount of work or knowledge could have succeeded. Also, large parts of Steins's work (e.g., the fundamental papers [27], [42], [48], [54], [65] on complex analysis in tube domains) don't fit any simple one-paragraph description such as the one above.

It follows that no single mathematician is competent to present an adequate survey of Stein's work. As I attempt the task, I am keenly aware that many of Stein's papers are incomprehensible to me, while others were of critical importance to my own work. Inevitably, therefore, my survey is biased, as any reader will see. Fortunately, S. Gindikin provided me with a layman's explanation of Stein's contributions to representation theory, thus keeping the bias (I hope) within reason. I am grateful to Gindikin for his help, and also to Y. Sagher for a valuable suggestion.

For purposes of this article, representation theory deals with the construction and classification of the irreducible unitary representations of a semisimple Lie group. Classical Fourier analysis starts with the $L^{p}$-boundedness of two fundamental operators, the maximal function

$$
f^{*}(x)=\sup _{h>0} \frac{1}{2 h} \int_{x-h}^{x+h}|f(y)| d y,
$$

and the Hilbert transform

$$
H f(x)=\lim _{\epsilon \rightarrow 0+} \frac{1}{\pi} \int_{|x-y|>\epsilon} \frac{f(x) d y}{x-y} .
$$

Finally, we shall be concerned with those problems in partial differential equations that come from several complex variables.

\section{I.1. Complex interpolation}

Let us begin with Stein's work on interpolation of operators. As background, we state and prove a classical result:

Theorem I.1.1 (Riesz convexity theorem). Suppose $X, Y$ are measure spaces, and suppose $T$ is an operator that carries functions on $X$ to functions on $Y$. Assume $T$ is bounded from $L^{p_{0}}(X)$ to $L^{r_{0}}(Y)$, and from $L^{p_{1}}(X)$ to $L^{r_{1}}(Y)$. (Here $p_{0}, p_{1}, r_{0}, r_{1} \in[1, \infty]$.) Then $T$ is bounded from $L^{p}(X)$ to $L^{r}(Y)$ for $\frac{1}{p}=\frac{t}{p_{1}}+\frac{(1-t)}{p_{0}}$, $\frac{1}{r}=\frac{t}{r_{1}}+\frac{(1-t)}{r_{0}}, 0 \leq t \leq 1$.

\footnotetext{
${ }^{1}$ Adapted from the article $\mathrm{Fe} 6$.
} 
The Riesz convexity theorem says that the points $\left(\frac{1}{p}, \frac{1}{r}\right)$ for which $T$ is bounded from $L^{p}$ to $L^{r}$ form a convex region in the plane. A standard application is the Hausdorff-Young inequality: We take $T$ to be the Fourier transform on $\mathbb{R}^{n}$, and note that $T$ is obviously bounded from $L^{1}$ to $L^{\infty}$, and from $L^{2}$ to $L^{2}$. Therefore, $T$ is bounded from $L^{p}$ to the dual class $L^{p^{\prime}}$ for $1 \leq p \leq 2$.

The idea of the proof of the Riesz convexity theorem is to estimate $\int_{Y}(T f) \cdot g$ for $f \in L^{p}$ and $g \in L^{r^{\prime}}$. Say $f=F e^{i \phi}$ and $g=G e^{i \psi}$ with $F, G \geq 0$ and $\phi, \psi$ real. Then we can define analytic families of functions $f_{z}, g_{z}$ by setting $f_{z}=F^{a z+b} e^{i \phi}$, $g_{z}=G^{c z+d} e^{i \psi}$, for real $a, b, c, d$ to be picked in a moment.

Define

$$
\Phi(z)=\int_{Y}\left(T f_{z}\right) g_{z}
$$

Evidently, $\Phi$ is an analytic function of $z$.

For the correct choice of $a, b, c, d$ we have

$$
\begin{array}{rlllrl}
\left|f_{z}\right|^{p_{0}}=|f|^{p} & \text { and } & \left|g_{z}\right|^{r_{0}^{\prime}}=|g|^{r^{\prime}} & \text { when } & \operatorname{Re} z=0 ; \\
\left|f_{z}\right|^{p_{1}}=|f|^{p} & \text { and } & \left|g_{z}\right|^{r_{1}^{\prime}}=|g|^{r^{\prime}} & \text { when } & \operatorname{Re} z=1 ; \\
f_{z}=f & \text { and } & g_{z}=g & & \text { when } & z=t .
\end{array}
$$

From (I.1.2) we see that $\left\|f_{z}\right\|_{L^{p_{0}}},\left\|g_{z}\right\|_{L^{r^{\prime}}{ }_{0}} \leq C$ for Re $z=0$. So the definition (I.1.1) and the assumption $T: L^{p_{0}} \rightarrow L^{r_{0}}$ show that

$$
|\Phi(z)| \leq C^{\prime} \text { for } \operatorname{Re} z=0 .
$$

Similarly, (I.1.3) and the assumption $T: L^{p_{1}} \rightarrow L^{r_{1}}$ imply

$$
|\Phi(z)| \leq C^{\prime} \text { for } \operatorname{Re} z=1 \text {. }
$$

Since $\Phi$ is analytic, (I.1.5) and (I.1.6) imply $|\Phi(z)| \leq C^{\prime}$ for $0 \leq \operatorname{Re} z \leq 1$ by the maximum principle for a strip. In particular, $|\Phi(t)| \leq C^{\prime}$. In view of (I.1.4), this means that $\left|\int_{Y}(T f) g\right| \leq C^{\prime}$, with $C^{\prime}$ determined by $\|f\|_{L^{p}}$ and $\|g\|_{L^{r^{\prime}}}$.

Thus, $T$ is bounded from $L^{p}$ to $L^{r}$, and the proof of the Riesz convexity theorem is complete.

This proof had been well known for over a decade, when Stein discovered an amazingly simple way to extend its usefulness by an order of magnitude. He realized that an ingenious argument by Hirschman [Hi] on certain multiplier operators on $L^{p}\left(\mathbb{R}^{n}\right)$ could be viewed as a Riesz convexity theorem for analytic families of operators. Here is the result.

Theorem I.1.2 (Stein interpolation theorem). Assume $T_{z}$ is an operator depending analytically on $z$ in the strip $0 \leq \operatorname{Re} z \leq 1$. Suppose $T_{z}$ is bounded from $L^{p_{0}}$ to $L^{r_{0}}$ when $\operatorname{Re} z=0$, and from $L^{p_{1}}$ to $L^{r_{1}}$ when $\operatorname{Re} z=1$. Then $T_{t}$ is bounded from $L^{p}$ to $L^{r}$, where $\frac{1}{p}=\frac{t}{p_{1}}+\frac{(1-t)}{p_{0}}, \frac{1}{r}=\frac{t}{r_{1}}+\frac{(1-t)}{r_{0}}$, and $0 \leq t \leq 1$.

Remarkably, the proof of the theorem comes from that of the Riesz convexity theorem by adding a single letter of the alphabet. Instead of taking $\Phi(z)=\int_{Y}\left(T f_{z}\right) g_{z}$ as in (1.1.1), we set $\Phi(z)=\int_{Y}\left(T_{z} f_{z}\right) g_{z}$. The proof of the Riesz convexity theorem applies with no further changes.

The Stein interpolation theorem is an essential tool that permeates modern Fourier analysis. Let me just give a single application here to illustrate what it can do. The example concerns Cesaro summability of multiple Fourier integrals. 
We define an operator $T_{\alpha R}$ on functions on $\mathbb{R}^{n}$ by setting

$$
\widehat{T_{\alpha R} f}(\xi)=\left(1-\frac{|\xi|^{2}}{R^{2}}\right)_{+}^{\alpha} \hat{f}(\xi) .
$$

Then

$$
\left\|T_{\alpha R} f\right\|_{L^{p}\left(\mathbb{R}^{n}\right)} \leq C_{\alpha p}\|f\|_{L^{p}\left(\mathbb{R}^{n}\right)}, \quad \text { if }\left|\frac{1}{p}-\frac{1}{2}\right| \leq \frac{\alpha}{n-1} .
$$

This follows immediately from the Stein interpolation theorem. We let $\alpha$ play the role of the complex parameter $z$, and we interpolate between the elementary cases $p=1$ and $p=2$. Inequality (I.1.7), due to Stein, was the first nontrivial progress on spherical summation of multiple Fourier series.

\section{I.2. Curvature And the Fourier transform}

One of the most fascinating themes in Fourier analysis in the last several decades has been the connection between the Fourier transform and curvature. Stein has pioneered this set of ideas and has made critical early discoveries. To illustrate, I will pick out two of his results. The first is a restriction theorem, i.e., a result on the restriction $\left.\hat{f}\right|_{\Gamma}$ of the Fourier transform of a function $f \in L^{p}\left(\mathbb{R}^{n}\right)$ to a set $\Gamma$ of measure zero. If $p>1$, then the standard inequality $\hat{f} \in L^{p^{\prime}}\left(\mathbb{R}^{n}\right)$ suggests that $\hat{f}$ should not even be well defined on $\Gamma$, since $\Gamma$ has measure zero. Indeed, if $\Gamma$ is (say) the $x$-axis in the plane $\mathbb{R}^{2}$, then we can easily find functions $f\left(x_{1}, x_{2}\right)=$ $\varphi\left(x_{1}\right) \psi\left(x_{2}\right) \in L^{p}\left(\mathbb{R}^{2}\right)$ for which $\left.\hat{f}\right|_{\Gamma}$ is infinite everywhere. Fourier transforms of $f \in L^{p}\left(\mathbb{R}^{2}\right)$ clearly cannot be restricted to straight lines. Stein proved that the situation changes drastically when $\Gamma$ is curved. His result is as follows.

Theorem I.2.1 (Stein restriction theorem). Suppose $\Gamma$ is the unit circle, $1 \leq p<$ $\frac{8}{7}$, and $f \in C_{0}^{\infty}\left(\mathbb{R}^{2}\right)$. Then we have the a priori inequality $\left\|\left.\hat{f}\right|_{\Gamma}\right\|_{L^{2}} \leq C_{p}\|f\|_{L^{p}\left(\mathbb{R}^{2}\right)}$, with $C_{p}$ depending only on $p$.

Using this a priori inequality, we can trivially pass from the dense subspace $C_{0}^{\infty}$ to define the operator $\left.f \mapsto \hat{f}\right|_{\Gamma}$ for all $f \in L^{p}\left(\mathbb{R}^{2}\right)$. Thus, the Fourier transform of $f \in L^{p}\left(p<\frac{8}{7}\right)$ may be restricted to the unit circle.

Improvements and generalizations were soon proven by other analysts, but it was Stein who first demonstrated the phenomenon of restriction of Fourier transforms.

Stein's proof of his restriction theorem is amazingly simple. If $\mu$ denotes uniform measure on the circle $\Gamma \subset \mathbb{R}^{2}$, then for $f \in C_{0}^{\infty}$ we have

$$
\int_{\Gamma}|\hat{f}|^{2}=\int_{\mathbb{R}^{2}}(\hat{f} \mu) \overline{(\hat{f})}=\langle f * \hat{\mu}, f\rangle \leq\|f\|_{L^{p}}\|f * \hat{\mu}\|_{L^{p^{\prime}}} .
$$

The Fourier transform $\hat{\mu}(\xi)$ is a Bessel function. It decays like $|\xi|^{-\frac{1}{2}}$ at infinity, a fact intimately connected with the curvature of the circle. In particular, $\hat{\mu} \in L^{q}$ for $4<q \leq \infty$, and therefore $\|f * \hat{\mu}\|_{L^{p^{\prime}}} \leq C_{p}\|f\|_{L^{p}}$ for $1 \leq p<\frac{8}{7}$, by the usual elementary estimates for convolutions. Putting this estimate back into (I.2.1), we see that $\int_{\Gamma}|\hat{f}|^{2} \leq C_{p}\|f\|_{L^{p}}^{2}$, which proves the Stein restriction theorem. The Stein restriction theorem means a lot to me personally, and has strongly influenced my own work in Fourier analysis.

The second result of Stein's relating the Fourier transform to curvature concerns the differentiation of integrals on $\mathbb{R}^{n}$. 
Theorem I.2.2. Suppose $f \in L^{p}\left(\mathbb{R}^{n}\right)$ with $n \geq 3$ and $p>\frac{n}{n-1}$. For $x \in \mathbb{R}^{n}$ and $r>0$, let $F(x, r)$ denote the average of $f$ on the sphere of radius $r$ centered at $x$. Then $\lim _{r \rightarrow 0} F(x, r)=f(x)$ almost everywhere.

The point is that unlike the standard Lebesgue theorem, we are averaging $f$ over a small sphere instead of a small ball. As in the restriction theorem, we are seemingly in trouble because the sphere has measure zero in $\mathbb{R}^{n}$, but the curvature of the sphere saves the day. This theorem is obviously closely connected to the smoothness of the solutions of the wave equation.

The proof of the above differentiation theorem relies on an elementary Tauberian theorem:

Suppose that $\lim _{R \rightarrow 0} \frac{1}{R} \int_{0}^{R} F(r) d r$ exists and $\int_{0}^{\infty} r\left|\frac{d F}{d r}\right|^{2} d r<\infty$.

Then $\lim _{R \rightarrow 0} F(R)$ exists, and equals $\lim _{R \rightarrow 0} \frac{1}{R} \int_{0}^{R} F(r) d r$.

This result had long been used, e.g., to pass from Cesaro averages of Fourier series to partial sums. (See Zygmund [Zy1.) On more than one occasion, Stein has shown the surprising power hidden in the elementary Tauberian theorem. Here we apply it to $F(x, r)$ for a fixed $x$. In fact, we have $F(x, r)=\int f(x+r y) d \mu(y)$, with $\mu$ equal to normalized surface measure on the unit sphere, so that the Fourier transforms of $F$ and $f$ are related by $\hat{F}(\xi, r)=\hat{f}(\xi) \hat{\mu}(r \xi)$ for each fixed $r$. Therefore, assuming $f \in L^{2}$ for simplicity, we obtain

$$
\begin{aligned}
\int_{\mathbb{R}^{n}} & \left(\int_{0}^{\infty} r\left|\frac{\partial}{\partial r} F(x, r)\right|^{2} d r\right) d x=\int_{0}^{\infty} r\left[\int_{\mathbb{R}^{n}}\left|\frac{\partial}{\partial r} F(x, r)\right|^{2} d x\right] d r \\
& =\int_{0}^{\infty} r\left[\int_{\mathbb{R}^{n}}\left|\frac{\partial}{\partial r} \widehat{F}(\xi, r)\right|^{2} d \xi\right] d r=\int_{\mathbb{R}^{n}} \int_{0}^{\infty} r\left|\frac{\partial}{\partial r} \hat{\mu}(r \xi)\right|^{2}|\hat{f}(\xi)|^{2} d r d \xi \\
& \left.=\int_{\mathbb{R}^{n}}\left\{\int_{0}^{\infty} r\left|\frac{\partial}{\partial r} \hat{\mu}(r \xi)\right|^{2} d r\right\}|\hat{f}(\xi)|^{2} d \xi=\text { (const. }\right) \int_{\mathbb{R}^{n}}|\hat{f}(\xi)|^{2} d \xi<\infty .
\end{aligned}
$$

(Here we make crucial use of curvature, which causes $\hat{\mu}$ to decay at infinity, so that the integral in curly braces converges.) It follows that $\int_{0}^{\infty} r\left|\frac{\partial}{\partial r} F(x, r)\right|^{2} d r<\infty$ for almost every $x \in \mathbb{R}^{n}$. On the other hand, $\frac{1}{R} \int_{0}^{R} F(x, r) d r$ is easily seen to be the convolution of $f$ with a standard approximate identity. Hence the usual Lebesgue differentiation theorem shows that $\lim _{R \rightarrow 0} \frac{1}{R} \int_{0}^{R} F(x, r) d r=f(x)$ for almost every $x$.

So for almost all $x \in \mathbb{R}^{n}$, the function $F(x, r)$ satisfies the hypotheses of the elementary Tauberian theorem. Consequently,

$$
\lim _{r \rightarrow 0} F(x, r)=\lim _{R \rightarrow 0} \frac{1}{R} \int_{0}^{R} F(x, r) d r=f(x)
$$

almost everywhere, proving the Stein differentiation theorem for $f \in L^{2}\left(\mathbb{R}^{n}\right)$.

To prove the full result for $f \in L^{p}\left(\mathbb{R}^{n}\right), p>\frac{n}{n-1}$, we repeat the above argument with surface measure $\mu$ replaced by an even more singular distribution on $\mathbb{R}^{n}$. Thus we obtain a stronger conclusion than asserted, when $f \in L^{2}$. On the other hand, for $f \in L^{1+\epsilon}$ we have a weaker result than that of Stein, namely the Lebesgue differentiation theorem. Interpolating between $L^{2}$ and $L^{1+\epsilon}$, one obtains the Stein differentiation theorem.

The two results we picked out here are only a sample of the work of Stein and others on curvature and the Fourier transform. For instance, J. Bourgain 
has dramatic results on both the restriction problem and spherical averages. See sections $\amalg .7$ and $\amalg .9$ in Part $\amalg$ for further results connected to these topics.

\section{I.3. $H^{p}$-SPACES}

Another essential part of Fourier analysis is the theory of $H^{p}$-spaces. Stein transformed the subject twice, once in a joint paper with Guido Weiss, and again in a joint paper with me. Let us start by recalling how the subject looked before Stein's work. The classical theory deals with analytic functions $F(z)$ on the unit disc. Recall that $F$ belongs to $H^{p}(0<p<\infty)$ if the norm $\|F\|_{H^{p}} \equiv$ $\lim _{r \rightarrow 1_{-}}\left(\int_{0}^{2 \pi}\left|F\left(r e^{i \theta}\right)\right|^{p} d \theta\right)^{\frac{1}{p}}$ is finite.

The classical $H^{p}$-spaces serve two main purposes. First, they provide growth conditions under which an analytic function tends to boundary values on the unit circle. Secondly, $H^{p}$ serves as a substitute for $L^{p}$ to allow basic theorems on Fourier series to extend from $1<p<\infty$ to all $p>0$. To prove theorems about $F \in H^{p}$, the main tool is the Blaschke product

$$
B(z)=\prod_{\nu} e^{i \theta_{\nu}} \frac{z_{\nu}-z}{1-\bar{z}_{\nu} z}
$$

where $\left\{z_{\nu}\right\}$ are the zeroes of the analytic function $F$ in the disc, and $\theta_{\nu}$ are suitable phases. The point is that $B(z)$ has the same zeroes as $F$, yet it has absolute value 1 on the unit circle. We illustrate the role of the Blaschke product by sketching the proof of the Hardy-Littlewood maximal theorem for $H^{p}$. The maximal theorem says that $\left\|F^{*}\right\|_{L^{p}} \leq C_{p}\|F\|_{H^{p}}$ for $0<p<\infty$, where $F^{*}(\theta)=\sup _{z \in \Gamma(\theta)}|F(z)|$, and $\Gamma(\theta)$ is the convex hull of $e^{i \theta}$ and the circle of radius $\frac{1}{2}$ about the origin.

This basic result is closely connected to the pointwise convergence of $F(z)$ as $z \in \Gamma(\theta)$ tends to $e^{i \theta}$. To prove the maximal theorem, we argue as follows.

First suppose $p>1$. Then we don't need analyticity of $F$. We can merely assume that $F$ is harmonic and deduce the maximal theorem from real variables. In fact, it is easy to show that $F$ arises as the Poisson integral of an $L^{p}$ function $f$ on the unit circle. The maximal theorem for $f$, a standard theorem of real variables, says that $\|M f\|_{L^{p}} \leq C_{p}\|f\|_{L^{p}}$, where $M f(\theta)=\sup _{h>0}\left(\frac{1}{2 h} \int_{\theta-h}^{\theta+h}|f(x)| d t\right)$. It is quite simple to show that $F^{*}(\theta)<C M f(\theta)$. Therefore $\left\|F^{*}\right\|_{L^{p}} \leq C\|M f\|_{L^{p}}<C^{\prime}\|F\|_{H^{p}}$, and the maximal theorem is proven for $H^{p}(p>1)$.

If $p \leq 1$, then the problem is more subtle, and we need to use analyticity of $F(z)$. Assume for a moment that $F$ has no zeroes in the unit disc. Then for $0<q<p$, we can define a single-valued branch of $(F(z))^{q}$, which will belong to $H^{\frac{p}{q}}$ since $F \in H^{p}$. Since $\tilde{p} \equiv \frac{p}{q}>1$, the maximal theorem for $H^{\tilde{p}}$ is already known. Hence, $\max _{z \in \Gamma(\theta)}\left|(F(z))^{q}\right| \in L^{\frac{p}{q}}$, with norm

$$
\int_{-\pi}^{\pi}\left(\max _{z \in \Gamma(\theta)}\left|(F(z))^{q}\right|\right)^{\frac{p}{q}} d \theta \leq C_{p, q}\left\|F^{q}\right\|_{H^{\frac{p}{q}}}^{\frac{p}{q}}=C_{P, q}\|F\|_{H^{p}}^{p} .
$$

That is, $\left\|F^{*}\right\|_{L^{p}} \leq C_{p, q}\|F\|_{H^{p}}$, proving the maximal theorem for functions without zeroes.

To finish the proof, we must deal with the zeroes of an $F \in H^{p}(p \leq 1)$. We bring in the Blaschke product $B(z)$, as in (I.3.1). Since $B(z)$ and $F(z)$ have the same zeroes and since $|B(z)|=1$ on the unit circle, we can write $F(z)=G(z) B(z)$ with $G$ analytic, and $|G(z)|=|F(z)|$ on the unit circle. Thus, $\|G\|_{H^{p}}=\|F\|_{H^{p}}$. 
Inside the circle, $G$ has no zeroes and $|B(z)| \leq 1$. Hence $|F| \leq|G|$, so

$$
\left\|\max _{z \in \Gamma(\theta)}|F(z)|\right\|_{L^{p}} \leq\left\|\max _{z \in \Gamma(\theta)}|G(z)|\right\|_{L^{p}} \leq C_{p}\|G\|_{H^{p}}=C_{p}\|F\|_{H^{p}}
$$

by the maximal theorem for functions without zeroes. The proof of the maximal theorem is complete. (We have glossed over difficulties that should not enter an expository paper.)

Classically, $H^{p}$ theory works only in one complex variable, so it is useful only for Fourier analysis in one real variable. Attempts to generalize $H^{p}$ to several complex variables ran into a lot of trouble because the zeroes of an analytic function $F\left(z_{1} \cdots z_{n}\right) \in H^{p}$ form a variety $V$ with growth conditions. Certainly $V$ is much more complicated than the discrete set of zeroes $\left\{z_{\nu}\right\}$ in the disc. There is no satisfactory substitute for the Blaschke product. For a long time, this blocked all attempts to extend the deeper properties of $H^{p}$ to several variables.

Stein and Weiss [13] realized that several complex variables was the wrong generalization of $H^{p}$ for purposes of Fourier analysis. They kept clearly in mind what $H^{p}$-spaces are supposed to do, and they kept an unprejudiced view of how to achieve it. They found a version of $H^{p}$ theory that works in several variables.

The idea of Stein and Weiss was very simple. They viewed the real and imaginary parts of an analytic function on the disc as the gradient of a harmonic function. In several variables, the gradient of a harmonic function is a system $\vec{u}=\left(u_{1}, u_{2}, \ldots, u_{n}\right)$ of functions on $\mathbb{R}^{n}$ that satisfies the Stein-Weiss CauchyRiemann equations

$$
\frac{\partial u_{j}}{\partial x_{k}}=\frac{\partial u_{k}}{\partial x_{j}}, \quad \sum_{k} \frac{\partial u_{k}}{\partial x_{k}}=0 .
$$

In place of the Blaschke product, Stein and Weiss used the following simple observation. If $\vec{u}=\left(u_{1} \cdots u_{n}\right)$ satisfies (1.3.2), then $|\vec{u}|^{p}=\left(u_{1}^{2}+u_{2}^{2}+\cdots+u_{n}^{2}\right)^{\frac{p}{2}}$ is subharmonic for $p>\frac{n-2}{n-1}$. We sketch the simple proof of this fact, then explain how an $H^{p}$ theory can be founded on it.

To see that $|\vec{u}|^{p}$ is subharmonic, we first suppose $|\vec{u}| \neq 0$ and calculate $\Delta\left(|\vec{u}|^{p}\right)$ in coordinates that diagonalize the symmetric matrix $\left(\frac{\partial u_{j}}{\partial x_{k}}\right)$ at a given point. The result is

$$
\Delta\left(|\vec{u}|^{p}\right)=p|\vec{u}|^{p-2}\left\{|\vec{w}|^{2}|\vec{u}|^{2}-(2-p)|\vec{v}|^{2}\right\},
$$

with $w_{k}=\frac{\partial u_{k}}{\partial x_{k}}$ and $v_{k}=u_{k} w_{k}$.

Since $\sum_{k=1}^{n} w_{k}=0$ by the Cauchy-Riemann equations, we have

$$
\left|w_{k}\right|^{2}=\left|\sum_{j \neq k} w_{j}\right|^{2} \leq(n-1) \sum_{j \neq k}\left|w_{j}\right|^{2}=(n-1)|\vec{w}|^{2}-(n-1)\left|w_{k}\right|^{2},
$$

i.e., $\left|w_{k}\right|^{2} \leq \frac{n-1}{n}|\vec{w}|^{2}$. Hence $|\vec{v}|^{2} \leq\left(\max _{k}\left|w_{k}\right|^{2}\right)|\vec{u}|^{2} \leq\left(\frac{n-1}{n}\right)|\vec{w}|^{2}|\vec{u}|^{2}$, so the expression in curly braces in (I.3.3) is nonnegative for $p \geq \frac{n-2}{n-1}$, and $|\vec{u}|^{p}$ is subharmonic.

So far, we know that $|\vec{u}|^{p}$ is subharmonic where it isn't equal to zero. Hence for $0<r<r(x)$ we have

$$
|\vec{u}(x)|^{p} \leq A v_{|y-x|=r}|\vec{u}(y)|^{p},
$$


provided $|\vec{u}(x)| \neq 0$. However, (I.3.4) is obvious when $|\vec{u}(x)|=0$, so it holds for any $x$. That is, $|\vec{u}|^{p}$ is a subharmonic function for $p \geq \frac{n-2}{n-1}$, as asserted.

Now let us see how to build an $H^{p}$ theory for Cauchy-Riemann systems, based on subharmonicity of $|\vec{u}|^{p}$. To study functions on $\mathbb{R}^{n-1}(n \geq 2)$, we regard $\mathbb{R}^{n-1}$ as the boundary of $\mathbb{R}_{+}^{n}=\left\{\left(x_{1} \cdots x_{n}\right) \mid x_{n}>0\right\}$, and we define $H^{p}\left(\mathbb{R}_{+}^{n}\right)$ as the space of all Cauchy-Riemann systems $\left(u_{1}, u_{2}, \ldots, u_{n}\right)$ for which the norm

$$
\|\vec{u}\|_{H^{p}}^{p}=\sup _{t>0} \int_{\mathbb{R}^{n-1}}\left|\vec{u}\left(x_{1} \cdots x_{n-1}, t\right)\right|^{p} d x_{1} \cdots d x_{n-1}
$$

is finite. For $n=2$ this definition agrees with the usual $H^{p}$-spaces for the upper half-plane.

Next we show how the Hardy-Littlewood maximal theorem extends from the disc to $\mathbb{R}_{+}^{n}$.

Define the maximal function $M(\vec{u})(x)=\sup _{|y-x|<t}|\vec{u}(y, t)|$ for $x \in \mathbb{R}^{n-1}$. Then for $\vec{u} \in H^{p}\left(\mathbb{R}_{+}^{n}\right), \frac{n-2}{n-1}<p$, we have $M(\vec{u}) \in L^{p}\left(\mathbb{R}^{n-1}\right)$ with norm $\int_{\mathbb{R}^{n-1}}(M(\vec{u}))^{p} d x \leq$ $C\|\vec{u}\|_{H^{p}}^{p}$.

As in the classical case, the proof proceeds by reducing the problem to the maximal theorem for $L^{p}, p>1$. For small $h>0$, the function $F_{h}(x, t)=$ $|\vec{u}(x, t+h)|^{\frac{n-2}{n-1}},\left(x \in \mathbb{R}^{n-1}, t \geq 0\right)$ is subharmonic on $\mathbb{R}_{+}^{n}$ and continuous up to the boundary. Therefore,

$$
F_{h}(x, t) \leq \text { P.I. }\left(f_{h}\right),
$$

where P.I. is the Poisson integral and $f_{h}(x)=F_{h}(x, 0)=|\vec{u}(x, h)|^{\frac{n-2}{n-1}}$. By definition of the $H^{p}$-norm, we have

$$
\int_{\mathbb{R}^{n-1}}\left|f_{h}(x)\right|^{\tilde{p}} d x \leq\|\vec{u}\|_{H^{p}}^{p}, \text { with } \tilde{p}=\left(\frac{n-1}{n-2}\right) p>1 .
$$

One the other hand, since the Poisson integral arises by convolving with an approximate identity, one shows easily that

$$
\sup _{|y-x|<t} \text { P.I. }\left(f_{h}\right)(y, t) \leq C f_{h}^{*}(x)
$$

with

$$
f_{h}^{*}(x)=\sup _{r>0} r^{-(n-1)} \int_{|x-y|<r}\left|f_{h}(y)\right| d y\left(x \in \mathbb{R}^{n-1}\right) .
$$

The standard maximal theorem of real variables gives

$$
\int_{\mathbb{R}^{n-1}}\left(f_{h}^{*}\right)^{\tilde{p}} \leq C_{p} \int_{\mathbb{R}^{n-1}}\left|f_{h}\right|^{\tilde{p}},
$$

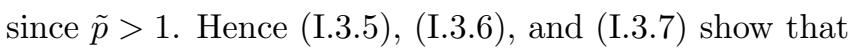

$$
\begin{gathered}
\int_{x \in \mathbb{R}^{n-1}}\left(\sup _{|y-x|<t} F_{h}(y, t)\right)^{\tilde{p}} d y \leq C_{p}\|\vec{u}\|_{H^{p}}^{p}, \text { i.e., } \\
\int_{x \in \mathbb{R}^{n-1}}\left(\sup _{|y-x|+h<t}|\vec{u}(y, t)|\right)^{p} d y \leq C_{p}\|\vec{u}\|_{H^{p}}^{p} .
\end{gathered}
$$

The constant $C_{p}$ is independent of $h$, so we can take the limit of (I.3.8) as $h \rightarrow 0$ to obtain the maximal theorem for $H^{p}$. The point is that subharmonicity of $|\vec{u}|^{\frac{n-2}{n-1}}$ substitutes for the Blaschke product in this argument. 
Stein and Weiss go on in [13] to obtain $n$-dimensional analogues of the classical theorems on existence of boundary values of $H^{p}$ functions. They also extend to $\mathbb{R}_{+}^{n}$ the classical F. and M. Riesz theorem on absolute continuity of $H^{1}$ boundary values. They begin the program of using $H^{p}\left(\mathbb{R}_{+}^{n}\right)$ in place of $L^{p}\left(\mathbb{R}^{n-1}\right)$, to extend the basic results of Fourier analysis to $p=1$ and below. We have seen how they deal with the maximal function. They prove also an $H^{p}$-version of the Sobolev theorem.

It is natural to try to get below $p=\frac{n-2}{n-1}$, and this can be done by studying higher gradients of harmonic functions in place of (I.3.2). See Calderón and Zygmund [CZ].

A joint paper [66] by Stein and me completed the task of developing basic Fourier analysis in the setting of the $H^{p}$-spaces. In particular, we showed in [66] that singular integral operators are bounded on $H^{p}\left(\mathbb{R}_{+}^{n}\right)$ for $0<p<\infty$. We proved this by finding a good viewpoint, and we found our viewpoint by repeatedly changing the definition of $H^{p}$. With each new definition, the function space $H^{p}$ remained the same, but it became clearer to us what was going on. Finally, we arrived at a definition of $H^{p}$ with the following excellent properties. First of all, it was easy to prove that the new definition of $H^{p}$ was equivalent to the Stein-Weiss definition and its extensions below $p=\frac{n-2}{n-1}$. Secondly, the basic theorems of Fourier analysis, which seemed very hard to prove from the original definition of $H^{p}\left(\mathbb{R}_{+}^{n}\right)$, became nearly obvious in terms of the new definition. Let me retrace the steps in [66].

Burkholder, Gundy, and Silverstein BGS] had shown that an analytic function $F=u+i v$ on the disc belongs to $H^{p}(0<p<\infty)$ if and only if the maximal function $u^{*}(\theta)=\sup _{z \in \Gamma(\theta)}|u(z)|$ belongs to $L^{p}$ (unit circle). Thus, $H^{p}$ can be defined purely in terms of harmonic functions $u$, without recourse to the harmonic conjugate $v$. Stein and I showed in [66] that the same thing happens in $n$ dimensions. That is, a Cauchy-Riemann system $\left(u_{1}, u_{2}, \ldots, u_{n}\right)$ on $\mathbb{R}_{+}^{n}$ belongs to the Stein-Weiss $H^{p_{-}}$ space $\left(p>\frac{n-2}{n-1}\right)$ if and only if the maximal function $u^{*}(x)=\sup _{|y-x|<t}\left|u_{n}(y, t)\right|$ belongs to $L^{p}\left(\mathbb{R}^{n-1}\right)$. (Here, the $n$th function $u_{n}$ plays a special role because $\mathbb{R}_{+}^{n}$ is defined by $\left\{x_{n}>0\right\}$.) Hence, $H^{p}$ may be viewed as a space of harmonic functions $u(x, t)$ on $\mathbb{R}_{+}^{n}$. The result extends below $p=\frac{n-2}{n-1}$ if we pass to higher gradients of harmonic functions.

The next step is to view $H^{p}$ as a space of distributions $f$ on the boundary $\mathbb{R}^{n-1}$. Any reasonable harmonic function $u(x, t)$ arises as a Poisson integral. Thus, it is natural to say that $f \in H^{p}\left(\mathbb{R}^{n-1}\right)$ if the maximal function

$$
f^{*}(x) \equiv \sup _{|y-x|<t}\left|\varphi_{t} * f(y)\right|
$$

belongs to $L^{p}$, where $\varphi_{t}$ is the Poisson kernel. Stein and I found in [66] that this definition is independent of the choice of the approximate identity $\varphi_{t}$, and that the grand maximal function

$$
\mathcal{M} f(x)=\sup _{\left\{\varphi_{t}\right\} \in \mathcal{A}} \sup _{|y-x|<t}\left|\varphi_{t} * f(y)\right|
$$

belongs to $L^{p}$, provided $f \in H^{p}$. Here $\mathcal{A}$ is a neighborhood of the origin in a suitable space of approximate identities. Thus, $f \in H^{p}$ if and only if $f^{*} \in L^{p}$ for some reasonable approximate identity. Equivalently, $f \in H^{p}$ if and only if the grand maximal function $\mathcal{M} f$ belongs to $L^{p}$. The proofs of these various equivalencies are not hard at all.

We have arrived at the good definition of $H^{p}$ mentioned above. 
To transplant basic Fourier analysis from $L^{p}(1<p<\infty)$ to $H^{p}(0<p<\infty)$, there is a simple algorithm. Take Calderón-Zygmund theory, and replace every application of the standard maximal theorem by an appeal to the grand maximal function. Only small changes are needed, and we omit the details here. Our paper [66] also contains the duality of $H^{1}$ and the bounded mean oscillation (BMO). Before leaving [66], let me mention an application of $H^{p}$ theory to $L^{p}$-estimates. If $\sigma$ denotes uniform surface measure on the unit sphere in $\mathbb{R}^{n}$, then $f \mapsto\left(\frac{\partial}{\partial x}\right)^{\alpha} \sigma * f$ is bounded on $L^{p}\left(\mathbb{R}^{n}\right)$, provided $n \geq 3$ and $\left|\frac{1}{p}-\frac{1}{2}\right| \leq \frac{1}{2}-\frac{\alpha}{n-1}$. Clearly, this result gives information on solutions to the wave equation.

The proof uses complex interpolation involving the analytic family of operators

$$
T_{\alpha}: f \mapsto(-\Delta)^{\frac{\alpha}{2}} \sigma * f \quad(\alpha \text { complex }),
$$

as is clear to anyone familiar with the Stein interpolation theorem. The trouble here is that $(-\Delta)^{\frac{\alpha}{2}}$ fails to be bounded on $L^{1}$ when $\alpha$ is imaginary. This makes it impossible to prove the sharp result $\left(\left|\frac{1}{p}-\frac{1}{2}\right|=\frac{1}{2}-\frac{\alpha}{n-1}\right)$ using $L^{p}$ alone. To overcome the difficulty, we use $H^{1}$ in place of $L^{1}$ in the interpolation argument. Imaginary powers of the Laplacian are singular integrals, which we know to be bounded on $H^{1}$. To show that complex interpolation works on $H^{1}$, we combined the duality of $H^{1}$ and BMO with the auxiliary function

$$
f^{\#}(x)=\sup _{Q \ni x} \frac{1}{|Q|} \int_{Q}\left|f(y)-\left(\operatorname{mean}_{Q} f\right)\right| d y .
$$

We refer the reader to [66] for an explanation of how to use $f^{\#}$, and for other applications.

After [66], Stein has proved a lot more theorems concerning $H^{p}$, both in higher rank settings and in contexts related to partial differential equations.

\section{I.4. The Cotlar-Stein lemma}

In section I.5 below we will meet an important singular integral operator (I.5.6) that acts on $L^{2}$ functions on a nilpotent Lie group $N$. In principle, $L^{2}$-boundedness of the translation-invariant operator (I.5.6) should be read off from the representation theory of $N$. In practice, representation theory provides a necessary and sufficient condition for $L^{2}$-boundedness that no one knows how to check. This fundamental analytic difficulty might have proved fatal. Fortunately, Stein had been working on a seemingly unrelated question and made a discovery that saved the day. Originally motivated by desire to get a simple proof of Calderón's theorem on commutator integrals Ca1, Stein proved a simple, powerful lemma in functional analysis. His contribution was to generalize to the critically important noncommutative case the remarkable lemma of Cotlar [Co. The Cotlar-Stein lemma turned out to be the perfect tool to prove $L^{2}$-boundedness of singular integrals on nilpotent groups.

The Coltar-Stein lemma deals with a sum $T=\sum_{\nu} T_{\nu}$ of operators on a Hilbert space. The idea is that if the $T_{\nu}$ are almost orthogonal, like projections onto the various coordinate axes, then the sum $T$ will have norm no larger than $\max _{\nu}\left\|T_{\nu}\right\|$. The precise statement is as follows. 
Theorem I.4.1 (The Cotlar-Stein lemma). Suppose $T=\sum_{k=1}^{M} T_{k}$ is a sum of operators on Hilbert space. Assume $\left\|T_{j}^{*} T_{k}\right\| \leq a(j-k)$ and $\left\|T_{j} T_{k}^{*}\right\| \leq a(j-k)$. Then $\|T\| \leq \sum_{-M}^{M} \sqrt{a(j)}$.

Proof. $\|T\|=\left(\left\|T T^{*}\right\|\right)^{\frac{1}{2}}$, so

$$
\begin{aligned}
\|T\|^{2 s} & \leq\left\|\left(T T^{*}\right)^{s}\right\|=\left\|\sum_{j_{1} \cdots j_{2 s}=1}^{M} T_{j_{1}} T_{j_{2}}^{*} \cdots T_{j_{2 s-1}} T_{j_{2 s}}^{*}\right\| \\
& \leq \sum_{j_{1} \cdots j_{2 s}=1}^{M}\left\|T_{j_{1}} T_{j_{2}}^{*} \cdots T_{j_{2 s-1}} T_{j_{2 s}}^{*}\right\| .
\end{aligned}
$$

We can estimate the summand in two different ways.

Writing $T_{j_{1}} T_{j_{2}}^{*} \cdots T_{j_{2 s-1}} T_{j_{2 s}}^{*}=\left(T_{j_{1}} T_{j_{2}}^{*}\right)\left(T_{j_{3}} T_{j_{4}}^{*}\right) \cdots\left(T_{j_{2 s-1}} T_{j_{2 s}}^{*}\right)$, we get

$$
\left\|T_{j_{1}} T_{j_{2}}^{*} \cdots T_{j_{2 s-1}} T_{j_{2}}^{*}\right\| \leq a\left(j_{1}-j_{2}\right) a\left(j_{3}-j_{4}\right) \cdots a\left(j_{2 s-1}-j_{2 s}\right) .
$$

One the other hand, writing

$$
T_{j_{1}} T_{j_{2}}^{*} \cdots T_{j_{2 s-1}} T_{j_{2 s}}^{*}=T_{j_{1}}\left(T_{j_{2}}^{*} T_{j_{3}}\right)\left(T_{j_{4}}^{*} T_{j_{5}}\right) \cdots\left(T_{j_{2 s-2}}^{*} T_{j_{2 s-1}}\right) T_{j_{2 s}}^{*},
$$

we see that

$$
\left\|T_{j_{1}} T_{j_{2}}^{*} \cdots T_{j_{2 s-1}} T_{j_{2 s}}^{*}\right\| \leq\left(\max _{j}\left\|T_{j}\right\|\right)^{2} a\left(j_{2}-j_{3}\right) a\left(j_{4}-j_{5}\right) \cdots a\left(j_{2 s-2}-j_{2 s-1}\right) .
$$

Taking the geometric mean of (I.4.2), (I.4.3) and putting the result into (I.4.1), we conclude that

$$
\begin{aligned}
\|T\|^{2 s} & \leq \sum_{j_{1} \cdots j_{2 s}=1}^{M}\left(\max _{j}\left\|T_{j}\right\|\right) \sqrt{a\left(j_{1}-j_{2}\right)} \sqrt{a\left(j_{2}-j_{3}\right)} \cdots \sqrt{a\left(j_{2 s-1}-j_{2 s}\right)} \\
& \leq\left(\max _{j}\left\|T_{j}\right\| \cdot M\right)\left(\sum_{\ell} \sqrt{a(\ell)}\right)^{2 s-1} .
\end{aligned}
$$

Thus, $\|T\| \leq\left(\max _{j}\left\|T_{j}\right\| \cdot M\right)^{\frac{1}{2 s}} \cdot\left(\sum_{\ell} \sqrt{a(\ell)}\right)^{\frac{2 s-1}{2 s}}$. Letting $s \rightarrow \infty$, we obtain the conclusion of the Cotlar-Stein lemma.

To apply the Cotlar-Stein lemma to singular integral operators, take a partition of unity $1=\sum_{\nu} \varphi_{\nu}(x)$ on $N$, so that each $\varphi_{\nu}$ is a dilate of a fixed $C_{0}^{\infty}$ function that vanishes in a neighborhood of the origin. Then $T: f \rightarrow K * f$ may be decomposed into a sum $T=\sum_{\nu} T_{\nu}$, with $T_{\nu}: f \rightarrow\left(\varphi_{\nu} K\right) * f$. The hypotheses of the Cotlar-Stein lemma are verified trivially, and the boundedness of singular integral operators follows. The $L^{2}$-boundedness of singular integrals on nilpotent groups is the Knapp-Stein theorem.

Almost immediately after this work, the Cotlar-Stein lemma became the standard method to prove $L^{2}$-boundedness of operators. Today one knows more, e.g., the $T(1)$ theorem of David and Journé. Still it is fair to say that the Cotlar-Stein lemma remains a key tool to establish $L^{2}$-boundedness.

Singular integrals on nilpotent groups were later applied by Stein in surprising ways in seemingly unrelated contexts. 


\section{I.5. REPRESENTATION THEORY}

Our next topic is the Kunze-Stein phenomenon, which links the Stein interpolation theorem to representations of Lie groups. For simplicity, we restrict our attention to $G=\mathrm{SL}(2, \mathbb{R})$, and begin by reviewing elementary Fourier analysis on $G$. The irreducible unitary representations of $G$ are as follows:

- the principal series parametrized by a sign $\sigma= \pm 1$ and a real parameter $t$;

- the discrete series parametrized by a $\operatorname{sign} \sigma= \pm 1$ and an integer $k \geq 0$; and

- the complementary series parametrized by a real number $t \in(0,1)$.

We don't need the full description of these representations here.

The irreducible representations of $G$ give rise to a Fourier transform. If $f$ is a function on $G$ and $U$ is an irreducible unitary representation of $G$, then we define

$$
\hat{f}(U)=\int_{G} f(g) U_{g} d g,
$$

where $d g$ denotes Haar measure on the group. Thus, $\hat{f}$ is an operator-valued function defined on the set of irreducible unitary representations of $G$. As in the Euclidean case, we can analyze convolutions in terms of the Fourier transform. In fact,

$$
\widehat{f * g}=\hat{f} \cdot \hat{g}
$$

as operators. Moreover, there is a Plancherel formula for $G$, which asserts that

$$
\|f\|_{L^{2}(G)}^{2}=\int\|\hat{f}(U)\|_{\text {Hilbert-Schmidt }}^{2} d \mu(U)
$$

for a measure $\mu$ (the Plancherel measure). The Plancherel measure for $G$ is known, but we don't need it here. However, we note that the complementary series has measure zero for the Plancherel measure.

These are, of course, analogues of familiar results in the elementary Fourier analysis of $\mathbb{R}^{n}$. Kunze and Stein discovered a fundamental new phenomenon in Fourier analysis on $G$ that has no analogue on $\mathbb{R}^{n}$. Their result is as follows.

Theorem I.5.1 (Kunze-Stein phenomenon). There exists a uniformly bounded representation $U_{\sigma, \tau}$ of $G$, parametrized by a sign $\sigma= \pm 1$ and a complex number $\tau$ in a strip $\Omega$, with the following properties:

(A) The $U_{\sigma, \tau}$ all act on the same Hilbert space $H$.

(B) For fixed $\sigma= \pm 1, g \in G$, and $\xi, \eta \in H$, the matrix element $\left\langle\left(U_{\sigma, \tau} g\right) \xi, \eta\right\rangle$ is an analytic function of $\tau \in \Omega$.

(C) The $U_{\sigma, \tau}$ for $\operatorname{Re} \tau=\frac{1}{2}$ are equivalent to the representations of the principal series.

(D) The $U_{+1, \tau}$ for suitable $\tau$ are equivalent to the representations of the complementary series.

(See [14] for the precise statement and proof as well as Ehrenpreis and Mautner [EM] for related results.)

The Kunze-Stein theorem suggests that analysis on $G$ resembles a fictional version of classical Fourier analysis in which the basic exponential $\xi \mapsto \exp (i \xi \cdot x)$ is a bounded analytic function on a strip $|\operatorname{Im} \xi| \leq C$, uniformly for all $x$.

As an immediate consequence of the Kunze-Stein theorem, we can give an analytic continuation of the Fourier transform for $G$. In fact, we set $\hat{f}(\sigma, \tau)=$ $\int_{G} f(g)\left(U_{\sigma, \tau}\right)_{g} d g$ for $\sigma= \pm 1, \tau \in \Omega$. 
Thus, $f \in L^{1}(G)$ implies $\hat{f}(\sigma, \cdot)$ analytic and bounded on $\Omega$. So we have continued analytically the restriction of $\hat{f}$ to the principal series. It is as if the Fourier transform of an $L^{1}$ function on $(-\infty, \infty)$ were automatically analytic in a strip. If $f \in L^{2}(G)$, then $\hat{f}(\sigma, \tau)$ is still defined on the line $\left\{\operatorname{Re} \tau=\frac{1}{2}\right\}$, by virtue of the Plancherel formula and part (C) of the Kunze-Stein theorem. Interpolating between $L^{1}(G)$ and $L^{2}(G)$ using the Stein interpolation theorem, we see that $f \in L^{p}(G)$ $(1 \leq p<2)$ implies $\hat{f}(\sigma, \cdot)$ analytic and satisfies an $L^{p^{\prime}}$-inequality on a strip $\Omega_{p}$. As $p$ increases from 1 to 2 , the strip $\Omega_{p}$ shrinks from $\Omega$ to the line $\left\{\operatorname{Re} \tau=\frac{1}{2}\right\}$. Thus we obtain the following result.

Corollary I.5.2. If $f \in L^{p}(G)(1 \leq p<2)$, then $\hat{f}$ is bounded almost everywhere with respect to the Plancherel measure.

Corollary I.5.3. For $1 \leq p<2$, we have the convolution inequality

$$
\|f * g\|_{L^{2}(G)} \leq C_{p}\|f\|_{L^{p}(G)}\|g\|_{L^{2}(G)} .
$$

To check Corollary $[.5 .2$ we look separately at the principal series, the discrete series, and the complementary series. For the principal series, we use the $L^{p^{\prime}}$ inequality established above for the analytic function $\tau \mapsto \hat{f}(\sigma, \tau)$ on the strip $\Omega_{p}$. Since an $L^{p}$-function analytic on a strip $\Omega_{p}$ is clearly bounded on an interior line $\left\{\operatorname{Re} \tau=\frac{1}{2}\right\}$, it follows at once that $\hat{f}$ is bounded on the principal series. Regarding the discrete series $U_{\sigma, k}$, we note that

$$
\left(\sum_{\sigma, k} \mu_{\sigma, k}\left\|\hat{f}\left(U_{\sigma, k}\right)\right\|^{p^{\prime}}\right)^{1 / p^{\prime}} \leq\|f\|_{L^{p}(G)}
$$

for suitable weights $\mu_{\sigma, k}$ and for $1 \leq p \leq 2$. The weights $\mu_{\sigma, k}$ amount to the Plancherel measure on the discrete series, and (1.5.2) is proved by a trivial interpolation, just like the standard Hausdorff-Young inequality. The boundedness of the $\left\|\hat{f}\left(U_{\sigma, k}\right)\right\|$ is immediate from (I.5.2). Thus the Fourier ransform $\hat{f}$ is bounded on both the principal series and the discrete series, for $f \in L^{p}(G)(1 \leq p<2)$. The complementary series has measure zero with respect to the Plancherel measure, so the proof of Corollary I.5.2 is complete. Corollary I.5.3 follows trivially from Corollary I.5.2, the Plancherel formula, and the elementary formula (I.5.1).

This proof of Corollary I.5.3 posed a significant challenge. Presumably, the corollary holds because the geometry of $G$ at infinity is so different from that of Euclidean space. For example, the volume of the ball of radius $R$ in $G$ grows exponentially as $R \rightarrow \infty$. This must have a profound impact on the way mass piles up when we take convolutions on $G$. On the other hand, the statement of Corollary I.5.3 clearly has nothing to do with cancellation; proving the corollary for two arbitrary functions $f, g$ is the same as providing it for $|f|$ and $|g|$. When we go back over the proof of Corollary I.5.3. we see cancellation used crucially (e.g., in the Plancherel formula for $G$ ), but there is no explicit mention of the geometry of $G$ at infinity. Clearly, there are subtle issues to explore regarding convolutions on $G$.

These basic issues were resolved in the case of semisimple groups $G$ of real rank 1 , by Ionescu [1o1,Io3] who proved a stronger Riesz-type rearrangement inequality of the form

$$
\int_{G} \int_{G} f(x) g\left(x^{\prime} x^{-1}\right) h\left(x^{\prime}\right) d x d x^{\prime} \leq C \int_{G} \int_{G} \widetilde{f}(x) \widetilde{g}\left(x^{\prime} x^{-1}\right) \widetilde{h}\left(x^{\prime}\right) d x d x^{\prime} .
$$


Here $f, g, h$ are, say, characterististic functions of measurable sets, $\tilde{f}, \widetilde{g}, \widetilde{h}$ are suitably defined bi-invariant (radial) nonincreasing rearrangements, and $C \geq 1$ is a constant that depends only on the group $G$.

The proof of (I.5.3) uses only real-variable techniques, such as the interplay of the Iwasawa and the Cartan decompositions of the group, consistent with the intuition described earlier that the Kunze-Stein inequality is a statement about nonnegative functions. The rearrangement inequality (I.5.3) implies the KunzeStein convolution inequality; so far, however, the inequality (I.5.3) is proved only when the semisimple group has real rank 1.

The Kunze-Stein phenomenon carries over to other semisimple groups, with profound consequences for representation theory. We explain briefly how the KunzeStein construction extends from $\mathrm{SL}(2, \mathbb{R})$ to more general semisimple Lie groups. The results we discuss are contained in the series of papers by Kunze and Stein [20], [22], [35], [69], Stein [38], [53], [77], and Knapp and Stein [47], [50], [51], [59], [64], [72], [76], [101], [105]. Let $G$ be a semisimple Lie group, and let $U^{\pi}$ be the unitary principal series representations of $G$ or one of its degenerate variants. The $U^{\pi}$ all act on a common Hilbert space, whose inner product we denote by $\langle\xi, \eta\rangle$. We needn't write down $U^{\pi}$ here nor even specify the parameters on which it depends. A finite group $W$, the Weyl group, acts on the parameters $\pi$ in such a way that the representations $U^{\pi}$ and $U^{w \pi}$ are unitarily equivalent for $w \in W$. Thus there is an intertwining operator $A(w, \pi)$ so that

$$
A(w, \pi) U_{g}^{w \pi}=U_{g}^{\pi} A(w, \pi) \text { for } g \in G, w \in W, \text { and for all } \pi .
$$

If $U^{\pi}$ is irreducible (which happens for most $\pi$ ), then $A(w, \pi)$ is uniquely determined by (I.5.4) up to multiplication by an arbitrary scalar $a(w, \pi)$. The crucial idea is as follows. If the $A(w, \pi)$ are correctly normalized (by the correct choice of $a(w, \pi)$ ), then $A(w, \pi)$ continues analytically to complex parameter values $\pi$. Moreover, for certain complex $(w, \pi)$, the quadratic form

$$
((\xi, \eta))_{w, \pi}=\langle(\text { TRIVIAL FACTOR }) A(w, \pi) \xi, \eta\rangle
$$

is positive definite.

In addition, the representation $U^{\pi}$ (defined for complex $\pi$ by a trivial analytic continuation) is unitary with respect to the inner product (I.5.5). Thus, starting with the principal series, we have constructed a new series of unitary representations of $G$. These new representations generalize the complementary series for $\operatorname{SL}(2, \mathbb{R})$. Applications of this basic construct are as follows.

(1) Starting with the unitary principal series, one obtains understanding of the previously discovered complementary series and construction of new ones, e.g., on $\operatorname{Sp}(4, \mathbb{C})$. Thus Stein exposed a gap in a supposedly complete list of complementary series representations of $\operatorname{Sp}(4, \mathbb{C})[\mathrm{GN}]$. See [38].

(2) Starting with a degenerate unitary principal series, Stein constructed new irreducible unitary representations of $\mathrm{SL}(n, \mathbb{C})$, in startling contradiction to the standard, supposedly complete list [GN] of irreducible unitary representations of that group. Much later, when the complete list of representations of $\operatorname{SL}(n, \mathbb{C})$ was given correctly, the representations constructed by Stein played an important role.

(3) The analysis of intertwining operators required to carry out analytic continuation also determines which exceptional values of $\pi$ lead to reducible 
principal series representations. For example, such reducible principal series representations exist already for $\operatorname{SL}(n, \mathbb{R})$, again contradicting what was "known". See Knapp and Stein [59].

A later theorem of Sahi and Stein [149] also fits into the same philosophy. In fact, Speh's representation can also be constructed by a more complicated variant of the analytic continuation defining the complementary series. Speh's representation plays an important role in the classification of the irreducible unitary representations of $\operatorname{SL}(2 n, \mathbb{R})$.

The main point of Stein's work in representation theory is thus to analyze the intertwining operators $A(w, \pi)$. In the simplest nontrivial case, $A(w, \pi)$ is a singular integral operator on a nilpotent group $N$. That is, $A(w, \pi)$ has the form

$$
T f(x)=\int_{N} K\left(x y^{-1}\right) f(y) d y
$$

with $K(y)$ smooth away from the identity and homogeneous of the critical degree with respect to dilations

$$
\left(\delta_{t}\right)_{t>0}: N \rightarrow N
$$

In (1.5.6),$d y$ denotes Haar measure on $N$. We know from the classical case $N=\mathbb{R}^{1}$ that (I.5.6) is a bounded operator only when the convolution kernel $K(y)$ satisfies a cancellation condition. Hence we assume $\int_{B_{1} \backslash B_{0}} K(y) d y=0$, where $B_{i}$ are dilates $\left(B_{i}=\delta_{t_{i}}(B)\right)$ of a fixed neighborhood of the identity in $N$.

It is crucial to show that such singular integrals are bounded on $L^{2}(N)$, generalizing the elementary $L^{2}$-boundedness of the Hilbert transform.

I.5.1. Further results. These ideas were expanded later in several directions. First, the Kunze-Stein convolution inequality (Corollary I.5.3) was proved in the full general case of noncompact semisimple Lie groups with finite center by Cowling $\mathrm{Cw}$.

Later, Stein and his collaborators used the representation theory of semisimple Lie groups to prove maximal and pointwise ergodic theorems. To state such a theorem, assume $G$ is a noncompact semisimple Lie group with finite center, $K$ is a maximal compact subgroup of $G$, and assume that $G$ acts ergodically by measure preserving transformations on a Borel probability space $(X, \mathfrak{B}, \mu)$.

For $t \geq 0$, let $\beta_{t}$ denote the probability measures with density $\left|B_{t}\right|^{-1} \mathbf{1}_{B_{t}}(g)$, where $B_{t}$ is the ball of radius $t$ in $G, B_{t}:=\{g \in G: d(g K, K) \leq t\}$. For $f \in L^{p}(X)$, $p \geq 1$, let

$$
\pi\left(\beta_{t}\right) f(x):=\int_{G} f\left(g^{-1} \cdot x\right) d \beta_{t}(g)
$$

denote the associated average operators. In a sequence of two papers [173], [184], Stein, Margulis, and Nevo proved pointwise ergodicity of the family of averages $\beta_{t}$, as well as a few other natural averages. This can be regarded as the natural analogue of the classical Wiener theorem in the setting of semisimple Lie groups:

Theorem I.5.4. With the definitions above, the family $\beta_{t}$ is a pointwise ergodic family in $L^{p}(X)$ for any $p>1$, i.e.,

$$
\lim _{t \rightarrow \infty} \pi\left(\beta_{t}\right) f(x)=\int_{X} f(x) d x
$$


for any $f \in L^{p}(X)$ and almost every $x \in X$. Moreover, one has the maximal inequality

$$
\left\|\sup _{t \in(0, \infty)}\left|\pi\left(\beta_{t}\right) f(x)\right|\right\|_{L^{p}(X)} \leq C_{p}\|f\|_{L^{p}(X)}, \quad p \in(1, \infty] .
$$

The main difficulty is that semisimple Lie groups are not amenable. Therefore, even though the standard maximal operator is bounded on $L^{p}(G / K), p>1$ (as a consequence of the Kunze-Stein convolution inequality 2 ) one cannot apply the classical Calderón transference principle in this situation to pass to ergodic theorems.

As a result, the proof of Theorem I.5.4 is considerably more complicated. In fact, it relies on the complete understanding of the representation theory of the group $G$, as described earlier. The contribution of the principal series is, essentially, already accounted for in the proof of the Kunze-Stein phenomenon. The main novelty is the analysis of the contribution of the complementary series, which requires new uniform estimates on the corresponding spherical functions.

\section{I.6. $\bar{\partial}$-PROBLEMS}

We prepare to discuss Stein's work on the $\bar{\partial}$-problems of several complex variables and related questions. Let us begin with the state of the subject before Stein's contributions. Suppose we are given a domain $D \subset \mathbb{C}^{n}$ with smooth boundary. If we try to construct analytic functions on $D$ with given singularities at the boundary, then we are led naturally to the following problems.

(I) Given a $(0,1)$-form $\alpha=\sum_{k=1}^{n} f_{k} \overline{d z_{k}}$ on $D$, find a function $u$ on $D$ that solves $\bar{\partial} u=\alpha$, where $\bar{\partial} u=\sum_{k=1}^{n} \frac{\partial u}{\partial \bar{z}_{k}} \overline{d z_{k}}$. Naturally, this is possible only if $\alpha$ satisfies the consistency condition $\bar{\partial} \alpha=0$, i.e., $\frac{\partial}{\partial \bar{z}_{k}} f_{j}=\frac{\partial}{\partial \bar{z}_{j}} f_{k}$. Moreover, $u$ is determined only modulo addition of an arbitrary analytic function on $D$. To make $u$ unique, we demand that $u$ be orthogonal to analytic functions in $L^{2}(D)$.

(II) There is a simple analogue of the $\bar{\partial}$-operator for functions defined only on the boundary $\partial D$. In local coordinates, we can easily find $(n-1)$ linearly independent complex vector fields $L_{1} \cdots L_{n-1}$ of type $(0,1)$ (i.e., $L_{j}=a_{j 1} \frac{\partial}{\partial \bar{z}_{1}}+a_{j 2} \frac{\partial}{\partial \bar{z}_{2}}+\cdots+a_{j_{n}} \frac{\partial}{\partial \bar{z}_{n}}$ for smooth, complex-valued $a_{j_{k}}$ ), whose real and imaginary parts are all tangent to $\partial D$. The restriction $u$ of an analytic function to $\partial D$ clearly satisfies $\bar{\partial}_{b} u=0$, where in local coordinates $\bar{\partial}_{b} u=\left(L_{1} u, L_{2} u, \ldots, L_{n-1} u\right)$. The boundary analogue of the $\bar{\partial}$-problem (I) is the inhomogeneous $\bar{\partial}_{b}$-equation $\bar{\partial}_{b} u=\alpha$. Again, this is possible only if $\alpha$ satisfies a consistency condition $\bar{\partial}_{b} \alpha=0$, and we impose the side condition that $u$ be orthogonal to analytic functions in $L^{2}(\partial D)$.

Just as analytic functions of one variable are related to harmonic functions, so the first-order systems (I) and (II) are related to equations involving the secondorder operators $\square$ and $\square_{b}$, the $\bar{\partial}$-Neumann and Kohn Laplacians. Both fall outside the scope of standard elliptic theory. Even for the simplest domains $D$, they posed a fundamental challenge to workers in the partial differential equations. More specifically, $\square$ is simply the Laplacian in the interior of $D$, but it is subject to nonelliptic boundary conditions. On the other hand, $\square_{b}$ is a nonelliptic system of

\footnotetext{
${ }^{2}$ In fact, it is also bounded from $L^{1}(G / K)$ to $L^{1, \infty}(G / K)$, due to the work of Strömberg [Sb].
} 
partial differential operators on $\partial D$, with no boundary conditions (since $\partial D$ has no boundary). Modulo lower-order terms (which, however, are important), $\square_{b}$ is the scalar operator $\mathcal{L}=\sum_{k=1}^{n-1}\left(X_{k}^{2}+Y_{k}^{2}\right)$, where $X_{k}$ and $Y_{k}$ are the real and imaginary parts of the basic complex vector fields $L_{k}$. At a given point in $\partial D$, the $X_{k}$ and $Y_{k}$ are linearly independent, but they don't span the tangent space of $\partial D$. This poses the danger that $\mathcal{L}$ will behave like a partial Laplacian such as $\Delta^{\prime}=\frac{\partial^{2}}{\partial x^{2}}+\frac{\partial^{2}}{\partial y^{2}}$ acting on functions $u(x, y, z)$. The equation $\Delta^{\prime} u=f$ is very bad. For instance, we can take $u(x, y, z)$ to depend on $z$ alone, so that $\Delta^{\prime} u=0$ with $u$ arbitrarily rough. Fortunately, $\mathcal{L}$ is more like the full Laplacian than like $\Delta^{\prime}$, because the $X_{k}$ and $Y_{k}$ together with their commutators $\left[X_{k}, Y_{k}\right]$ span the tangent space of $\partial D$ for suitable $D$. Thus, $\mathcal{L}$ is a well-behaved operator, thanks to the intervention of commutators of vector fields.

It was Kohn in the 1960s who proved the basic $C^{\infty}$ regularity theorems for $\square, \square_{b}$, $\bar{\partial}$, and $\bar{\partial}_{b}$ on strongly pseudoconvex domains (the simplest case). His proofs were based on subelliptic estimates such as $\left\langle\square_{b} w, w\right\rangle \geq c\|w\|_{(\epsilon)}^{2}-C\|w\|^{2}$ and brought to light the importance of commutators. Hörmander proved a celebrated theorem on $C^{\infty}$ regularity of operators,

$$
L=\sum_{j=1}^{N} X_{j}^{2}+X_{0},
$$

where $X_{0}, X_{1}, \ldots, X_{N}$ are smooth, real vector fields which, together with their repeated commutators, span the tangent space at every point.

If we allow $X_{0}$ to be a complex vector field, then we get a very hard problem that is not adequately understood to this day, except in very special cases.

Stein made a fundamental change in the study of the $\bar{\partial}$-problems by bringing in constructive methods. Today, thanks to the work of Stein with several collaborators, we know how to write down explicit solutions to the $\bar{\partial}$-problems modulo negligible errors on strongly pseudoconvex domains. Starting from these explicit solutions, it is then possible to prove sharp regularity theorems. Thus, the $\bar{\partial}$-equations on strongly pseudoconvex domains are understood completely. It is a major open problem to achieve comparable understanding of weakly pseudoconvex domains.

Now let us see how Stein and his coworkers were able to crack the strongly pseudoconvex case. We begin with the work of Folland and Stein [73]. The simplest example of a strongly psuedoconvex domain is the unit ball. Just as the disc is equivalent to the half-plane, the ball is equivalent to the Siegel domain $D_{\text {Siegel }}=$ $\left\{(z, w) \in \mathbb{C}^{n-1} \times\left.\mathbb{C}|\operatorname{Im} w>| z\right|^{2}\right\}$. Its boundary $H=\partial D_{\text {Siegel }}$ has an important symmetry group, including the following:

(a) Translations $(z, w) \mapsto(z, w) \cdot\left(z^{\prime}, w^{\prime}\right) \equiv\left(z+z^{\prime}, w+w^{\prime}+2 i z \cdot \bar{z}^{\prime}\right)$ for $\left(z^{\prime}, w^{\prime}\right) \in H$;

(b) Dilations $\partial_{t}:(z, w) \mapsto\left(t z, t^{2} w\right)$ for $t>0$;

(c) Rotations $(z, w) \mapsto(U z, w)$ for unitary $(n-1) \times(n-1)$ matrices $U$.

The multiplication law (a) makes $H$ into a nilpotent Lie group, the Heisenberg group. Translation-invariance of the Siegel domain allows us to pick the basic complex vector fields $L_{1} \cdots L_{n-1}$ to be translation-invariant on $H$. After we make a suitable choice of metric, the operators $\mathcal{L}$ and $\square_{b}$ become translation- and rotationinvariant, and homogeneous with respect to the dilations $\delta_{t}$. Therefore, the solution ${ }^{3}$ of $\square_{b} w=\alpha$ should have the form of a convolution $w=K * \alpha$ on the Heisenberg

\footnotetext{
${ }^{3}$ Kohn's work showed that $\square_{b} w=\alpha$ has a solution if we are in complex dimension $>2$. In two complex dimensions, $\square_{b} w=\alpha$ has no solution for most $\alpha$. We assume dimension $>2$ here.
} 
group. The convolution kernel $K$ is homogeneous with respect to the dilations $\delta_{t}$ and invariant under rotations. Also, since $K$ is a fundamental solution, it satisfies $\square_{b} K=0$ away from the origin. This reduces to an elementary ODE after we take the dilation- and rotation-invariance into account. Hence one can easily find $K$ explicitly and thus solve the $\square_{b}$-equation for the Siegel domain. To derive sharp regulatory theorems for $\square_{b}$, we combine the explicit fundamental solution with the Knapp-Stein theorem on singular integrals on the Heisenberg group. For instance, if $\square_{b} w \in L^{2}$, then $L_{j} L_{k} w, \bar{L}_{j} L_{k} w, L_{j} \bar{L}_{k} w$, and $\bar{L}_{j} \bar{L}_{k} w$ all belong to $L^{2}$. To see this, we write

$$
\square_{b} w=\alpha, \quad w=K * \alpha, \quad L_{j} L_{k} w=\left(L_{j} L_{k} K\right) * \alpha,
$$

and note that $L_{j} L_{k} K$ has the critical homogeneity and integral 0 . Thus $L_{j} L_{k} K$ is a singular integral kernel in the sense of Knapp and Stein, and it follows that $\left\|L_{j} L_{k} w\right\| \leq C\|\alpha\|$. For the first time, nilpotent Lie groups have entered into the study of $\bar{\partial}$-problems.

Folland and Stein viewed their results on the Heisenberg group not as ends in themselves, but rather as a tool to understand general strongly pseudoconvex Cauchy-Riemann (CR)-manifolds. A CR-manifold $M$ is a generalization of the boundary of a smooth domain $D \subset \mathbb{C}^{n}$. For simplicity, we will take $M=\partial D$ here. The key idea is that near any point $w$ in a strongly pseudoconvex $M$, the CR-structure for $M$ is very nearly equivalent to that of the Heisenberg group $H$ via a change of coordinates $\Theta_{w}: M \rightarrow H$. More precisely, $\Theta_{w}$ carries $w$ to the origin, and it carries the CR-structure on $M$ to a CR-structure on $H$ that agrees with the usual one at the origin. Therefore, if $w=K * \alpha$ is our known solution of $\square_{b} w=\alpha$ on the Heisenberg group, then it is natural to try

$$
w(z)=\int_{M} K\left(\Theta_{w}(z)\right) \alpha(w) d w
$$

as an approximate solution of $\square_{b} w=\alpha$ on $M$. (Since $w$ and $\alpha$ are sections of bundles, one has to explain carefully what (I.6.1) really means.) If we apply $\square_{b}$ to the $w$ defined by (I.6.1), then we find that

$$
\square_{b} w=\alpha-\mathcal{E} \alpha,
$$

where $\mathcal{E}$ is a sort of Heisenberg version of $(-\Delta)^{-\frac{1}{2}}$. In particular, $\mathcal{E}$ gains smoothness, so that $(I-\mathcal{E})^{-1}$ can be constructed modulo infinitely smoothing operators by means of a Neumann series. Therefore (1.6.1) and (1.6.2) show that the full solution of $\square_{b} w=\alpha$ is given (modulo infinitely smoothing errors) by

$$
w(z)=\sum_{k} \int_{M} K\left(\Theta_{w}(z)\right)\left(\mathcal{E}^{k} \alpha\right)(w) d w,
$$

from which one can deduce sharp estimates to understand completely $\square_{b}^{-1}$ on $M$.

The process is analogous to the standard method of freezing coefficients to solve variable-coefficient elliptic differential equations. Let us see how the sharp results are stated. As on the Heisenberg group, there are smooth, complex vector fields $L_{k}$ that span the tangent vectors of type $(0,1)$ locally. Let $X_{j}$ be the real and imaginary parts of the $L_{k}$. In terms of the $X_{j}$ we define non-Euclidean versions of standard geometric and analytic concepts. Thus, the non-Euclidean ball $\mathbb{B}(z, \rho)$ may be defined as an ellipsoid with principal axes of length $\sim \rho$ in the codimension- 1 hyperplane spanned by the $X_{j}$, and length $\sim \rho^{2}$ perpendicular to that hyperplane. In terms of $\mathbb{B}(z, \rho)$, the non-Euclidean Lipschitz spaces $\Gamma_{\alpha}(M)$ are defined as the 
set of functions $u$ for which $|u(z)-u(w)|<C \rho^{\alpha}$ for $w \in \mathbb{B}(z, \rho)$. (Here, $0<\alpha<1$. There is a natural extension to all $\alpha>0$.) The non-Euclidean Sobolev spaces $S_{m, p}(M)$ consist of all distributions $u$ for which all $X_{j_{1}} X_{j_{2}} \cdots X_{j_{s}} u \in L^{p}(M)$ for $0 \leq s \leq m$.

Then the sharp results on $\square_{b}$ are as follows. If $\square_{b} w=\alpha$ and $\alpha \in S_{m, p}(M)$, then $w \in S_{m+2, p}(M)$ for $m \geq 0,1<p<\infty$. If $\square_{b} w=\alpha$ and $\alpha \in \Gamma_{\alpha}(M)$, then $X_{j} X_{k} w \in \Gamma_{\alpha}(M)$ for $0<\alpha<1$ (say). For additional sharp estimates, and for comparisons between the non-Euclidean and standard function spaces, we refer the reader to [73].

To prove their sharp results, Folland and Stein developed the theory of singular integral operators in a non-Euclidean context. The Cotlar-Stein lemma proves the crucial results on $L^{2}$-boundedness of singular integrals. Additional difficulties arise from the noncommutativity of the Heisenberg group. In particular, standard singular integrals or pseudodifferential operators commute modulo lower-order errors, but non-Euclidean operators are far from commuting. This makes more difficult the passage from $L^{p}$-estimates to the Sobolev spaces $S_{m, p}(M)$.

Before we continue with Stein's work on $\bar{\partial}$, let me explain the remarkable paper of Rothschild and Stein [80]. It extends the Folland-Stein results and viewpoint to general Hörmander operators $\mathcal{L}=\sum_{j=1}^{N} X_{j}^{2}+X_{0}$. Actually, [80] deals with systems whose second-order part is $\sum_{j} X_{j}^{2}$, but for simplicity we restrict our attention here to $\mathcal{L}$. In explaining the proofs, we simplify even further by supposing $X_{0}=0$. The goal of the Rothschild-Stein paper is to use nilpotent groups to write down an explicit parametrix for $\mathcal{L}$ and prove sharp estimates for solutions of $\mathcal{L} u=f$. This ambitious hope is seemingly dashed at once by elementary examples. For instance, take $\mathcal{L}=X_{1}^{2}+X_{2}^{2}$ with

$$
X_{1}=\frac{\partial}{\partial x}, \quad X_{2}=x \frac{\partial}{\partial y} \text { on } \mathbb{R}^{2} .
$$

Then $X_{1}$ and $\left[X_{1}, X_{2}\right]$ span the tangent space, yet $\mathcal{L}$ clearly cannot be approximated by translation-invariant operators on a nilpotent Lie group in the sense of Folland and Stein. The trouble is that $\mathcal{L}$ changes character completely from one point to another. Away from the $y$-axis $\{x=0\}, \mathcal{L}$ is elliptic, so the only natural nilpotent group we can reasonably use is $\mathbb{R}^{2}$. On the $y$-axis, $\mathcal{L}$ degenerates and evidently cannot be approximated by a translation-invariant operator on $\mathbb{R}^{2}$. The problem is so obviously fatal, and its solution by Rothschild and Stein so simple and natural, that [80] must be regarded as a gem. Here is the idea:

Suppose we add an extra variable $t$ and lift $X_{1}$ and $X_{2}$ in (1.6.4) to vector fields

$$
\widetilde{X}_{1}=\frac{\partial}{\partial x}, \quad \widetilde{X}_{2}=x \frac{\partial}{\partial y}+\frac{\partial}{\partial t} \text { on } \mathbb{R}^{3} .
$$

Then the Hörmander operator $\widetilde{\mathcal{L}}=\widetilde{X}_{1}^{2}+\widetilde{X}_{2}^{2}$ looks the same at every point of $\mathbb{R}^{3}$, and may be readily understood in terms of nilpotent groups as in Folland and Stein [73]. In particular, one can essentially write down a fundamental solution and prove sharp estimates for $\widetilde{\mathcal{L}}^{-1}$. On the other hand, $\widetilde{\mathcal{L}}$ reduces to $\mathcal{L}$ when acting on functions $u(x, y, t)$ that do not depend on $t$. Hence, sharp results on $\widetilde{\mathcal{L}} u=f$ imply sharp results on $\mathcal{L} u=f$.

Thus we have the Rothschild-Stein program: First, add new variables and lift the given vector fields $X_{1} \cdots X_{N}$ to new vector fields $\widetilde{X}_{1} \cdots \widetilde{X}_{N}$ whose underlying 
structure does not vary from point to point. Next, approximate $\widetilde{\mathcal{L}}=\sum_{1}^{N} \widetilde{X}_{j}^{2}$ by a translation-invariant operator $\widehat{\mathcal{L}}=\sum_{1}^{N} Y_{j}^{2}$ on a nilpotent Lie group $\mathcal{N}$. Then analyze the fundamental solution of $\widehat{\mathcal{L}}$ and use it to write down an approximate fundamental solution for $\widetilde{\mathcal{L}}$. From the approximate solution, derive sharp estimates for solutions of $\widetilde{\mathcal{L}} u=f$. Finally, descend to the original equation $\mathcal{L} u=f$ by restricting our attention to functions $u, f$ that do not depend on the extra variables.

To carry out the first part of their program, Rothschild and Stein prove the following:

Theorem I.6.1. Let $X_{1} \cdots X_{N}$ be smooth vector fields on a neighborhood of the origin in $\mathbb{R}^{n}$. Assume that the $X_{j}$ and their commutators $\left[\left[\left[X_{j_{1}}, X_{j_{2}}\right], X_{j_{3}}\right] \cdots X_{j_{s}}\right]$ of order up to $r$ span the tangent space at the origin. Then we can find smooth vector fields $\widetilde{X}_{1} \cdots \widetilde{X}_{N}$ on a neighborhood $\widetilde{U}$ of the origin in $\mathbb{R}^{n+m}$ with the following properties:

(a) The $\widetilde{X}_{j}$ and their commutators up to order $r$ are linearly independent at each point of $\widetilde{U}$, except for the linear relations that follow formally from the antisymmetry of the bracket and the Jacobi identity.

(b) The $\widetilde{X}_{j}$ and their commutators up to order $r$ span the tangent space of $\widetilde{U}$.

(c) Acting on functions on $\mathbb{R}^{n+m}$ that do not depend on the last $m$ coordinates, the $\tilde{X}_{j}$ reduce to the given $X_{j}$.

Next we need a nilpotent Lie group $\mathcal{N}$ appropriate to the vector fields $\widetilde{X}_{1} \cdots \widetilde{X}_{N}$. The natural one is the free nilpotent group $\mathcal{N}_{N r}$ of step $r$ on $N$ generators. Its Lie algebra is generated by $Y_{1} \cdots Y_{N}$ whose Lie brackets of order higher than $r$ vanish, but whose brackets of order $\leq r$ are linearly independent, except for relations forced by antisymmetry of brackets and the Jacobi identity. We regard the $Y_{j}$ as translation-invariant vector fields on $\mathcal{N}_{N r}$. It is convenient to pick a basis $\left\{Y_{\alpha}\right\}_{\alpha \in A}$ for the Lie algebra of $\mathcal{N}_{N r}$, consisting of $Y_{1} \cdots Y_{N}$ and some of their commutators.

On $\mathcal{N}_{N r}$ we form the Hörmander operator $\widehat{\mathcal{L}}=\sum_{1}^{N} Y_{j}^{2}$. Then $\widehat{\mathcal{L}}$ is translationinvariant and homogeneous under the natural dilations on $\mathcal{N}_{N r}$. Hence $\widehat{\mathcal{L}}^{-1}$ is given by convolution on $\mathcal{N}_{N r}$ with a homogeneous kernel $K(\cdot)$ having a weak singularity at the origin. Hypoellipticity of $\widehat{\mathcal{L}}$ shows that $K$ is smooth away from the origin. Thus we understand the equation $\widehat{\mathcal{L}} u=f$ very well.

We want to use $\widehat{\mathcal{L}}$ to approximate $\widetilde{\mathcal{L}}$ at each point $y \in U$. To do so, we have to identify a neighborhood of $y$ in $\widetilde{U}$ with a neighborhood of the origin in $\mathcal{N}_{N r}$. This has to be done just right, or else $\widehat{\mathcal{L}}$ will fail to approximate $\widetilde{\mathcal{L}}$. The idea is to use exponential coordinates on both $\widetilde{U}$ and $\mathcal{N}_{N r}$. Thus, if $x=\exp \left(\sum_{\alpha \in A} t_{\alpha} Y_{\alpha}\right)$ (identity) $\in \mathcal{N}_{N r}$, then we use $\left(t_{\alpha}\right)_{\alpha \in A}$ as coordinates for $x$. Similarly, let $\left(\widetilde{X}_{\alpha}\right)_{\alpha \in A}$ be the commutators of $\widetilde{X}_{1} \cdots \widetilde{X}_{N}$ analogous to the $Y_{\alpha}$, and let $y \in \widetilde{U}$ be given. Then given a nearby point $x=\exp \left(\sum_{\alpha \in A} t_{\alpha} \widetilde{X}_{\alpha}\right) y \in \widetilde{U}$, we use $\left(t_{\alpha}\right)_{\alpha \in A}$ as coordinates for $x$.

Now we can identify $\widetilde{U}$ with a neighborhood of the identity in $\mathcal{N}_{N r}$, simply by identifying points with the same coordinates. Denote the identification by $\Theta_{y}: \widetilde{U} \rightarrow \mathcal{N}_{N r}$, and note that $\Theta_{y}(y)=$ identity.

In view of the identification $\Theta_{y}$, the operators $\widehat{\mathcal{L}}$ and $\widetilde{\mathcal{L}}$ live on the same space. The next step is to see that they are approximately equal. To formulate this, we need some bookkeeping on the nilpotent group $\mathcal{N}_{N r}$. Let $\left\{\delta_{t}\right\}_{t>0}$ be the natural 
dilations on $\mathcal{N}_{N r}$. If $\varphi \in C_{0}^{\infty}\left(\mathcal{N}_{N r}\right)$, then write $\varphi_{t}$ for the function $x \mapsto \varphi\left(\delta_{t} x\right)$. When $\varphi$ is fixed and $t$ is large, then $\varphi_{t}$ is supported in a tiny neighborhood of the identity. Let $\mathcal{D}$ be a differential operator acting on functions on $\mathcal{N}_{N r}$. We say that $\mathcal{D}$ has "degree" at most $k$ if for each $\varphi \in C_{0}^{\infty}\left(\mathcal{N}_{N r}\right)$ we have $\left|\mathcal{D}\left(\varphi_{t}\right)\right|=O\left(t^{k}\right)$ for large, positive $t$. According to this definition, $Y_{1}, \ldots, Y_{N}$ have degree 1 while $\left[Y_{j}, Y_{k}\right]$ has degree 2 , and the degree of $a(x)\left[Y_{j}, Y_{k}\right]$ depends on the behavior of $a(x)$ near the identity. Now we can say in what sense $\widetilde{\mathcal{L}}$ and $\widehat{\mathcal{L}}$ are approximately equal. The crucial result is as follows.

Theorem I.6.2. Under the map $\Theta_{y}^{-1}$, the vector field $\widetilde{X}_{j}$ pulls back to $Y_{j}+Z_{y, j}$, where $Z_{y, j}$ is a vector field on $\mathcal{N}_{N r}$ of degree $\leq 0$.

Using Theorem I.6.2 and the map $\Theta_{y}$, we can produce a parametrix for $\widetilde{\mathcal{L}}$ and prove that it works. In fact, we take

$$
\widetilde{K}(x, y)=K\left(\Theta_{y} x\right),
$$

where $K$ is the fundamental solution of $\widehat{\mathcal{L}}$. For fixed $y$, we want to know that

$$
\widetilde{\mathcal{L}} \widetilde{K}(x, y)=\delta_{y}(x)+\mathcal{E}(x, y),
$$

where $\delta_{y}(\cdot)$ is the Dirac delta-function and $\mathcal{E}(x, y)$ has only a weak singularity at $x=y$. To prove this, we use $\Theta_{y}$ to pull back to $\mathcal{N}_{N r}$. Recall that $\widetilde{\mathcal{L}}=\sum_{1}^{N} \tilde{X}_{j}^{2}$ while $\widehat{\mathcal{L}}=\sum_{1}^{N} Y_{j}^{2}$. Hence by Theorem I.6.2, $\widetilde{\mathcal{L}}$ pulls back to an operator of the form $\widehat{\mathcal{L}}+\mathcal{D}_{y}$ with $\mathcal{D}_{y}$ having degree at most 1 . Therefore (I.6.7) reduces to proving that

$$
\left(\widehat{\mathcal{L}}+\mathcal{D}_{y}\right) K(x)=\delta_{\text {id. }}(x)+\widehat{\mathcal{E}}(x),
$$

where $\widehat{\mathcal{E}}$ has only a weak singularity at the identity. Since $\widehat{\mathcal{L}} K(x)=\delta_{\text {id. }}(x),($ I.6.8) means simply that $\mathcal{D}_{y} K(x)$ has only a weak singularity at the identity. However, this is obvious from the smoothness and homogeneity of $K(x)$ and from the fact that $\mathcal{D}_{y}$ has degree $\leq 1$. Thus, $\widetilde{K}(x, y)$ is an approximate fundamental solution for $\widetilde{\mathcal{L}}$.

From the explicit fundamental solution for the lifted operator $\widetilde{\mathcal{L}}$, one can descend to deal with the original Hörmander operator $\mathcal{L}$ in two different ways.

(a) Prove sharp estimates for the lifted problem, then specialize to the case of functions that don't depend on the extra variables.

(b) Integrate out the extra variables from the fundamental solution for $\widetilde{\mathcal{L}}$ to obtain a fundamental solution for $\mathcal{L}$.

Rothschild and Stein used the first approach, (a). They succeeded in proving the estimate

$$
\begin{array}{r}
\left\|X_{0} u\right\|_{L^{p}(U)}+\left\|X_{j} X_{k} u\right\|_{L^{p}(U)} \leq C_{p}\left\|\left(\sum_{j=1}^{N} X_{j}^{2}+X_{0}\right) u\right\|_{L^{p}(V)}+C_{p}\|u\|_{L^{p}(V)} \\
\text { for } 1<p<\infty \text { and } U \Subset V .
\end{array}
$$

This is the most natural and the sharpest estimate for Hörmander operators. It was new even for $p=2$. Rothschild and Stein also proved sharp estimates in spaces analogous to the $\Gamma_{\alpha}$ and $S_{m, p}$ of Folland and Stein [73], as well as in 
standard Lipschitz and Sobolev spaces. We omit the details, but we point out that commuting derivatives past a general Hörmander operator here requires additional ideas.

Later, Nagel, Stein, and Wainger [128] returned to the second approach, (b), and were able to estimate the fundamental solution of a general Hörmander operator. This work overcomes substantial problems.

In fact, once we descend from the lifted problem to the original equation, we again face the difficulty that Hörmander operators cannot be modeled directly on nilpotent Lie groups. So it isn't even clear how to state a theorem on the fundamental solution of a Hörmander operator. Nagel, Stein, and Wainger [128] realized that a family of non-Euclidean balls $B_{\mathcal{L}}(x, \rho)$ associated to the Hörmander operator $\mathcal{L}$ plays the basic role. They defined the $B_{\mathcal{L}}(x, \rho)$ and proved their essential properties. In particular, they saw that the family of balls survives the projection from the lifted problem back to the original equation, even though the nilpotent Lie group structure is destroyed. Non-Euclidean balls had already played an important part in [73] by Folland and Stein. However, it was simple in [73] to guess the correct family of balls. For general Hörmander operators $\mathcal{L}$ the problem of defining and controlling non-Euclidean balls is much more subtle. Closely related results appear also in $[\mathrm{FP}$, and $[\mathrm{FS}$.

Let us look first at a nilpotent group such as $\mathcal{N}_{N r}$, with its family of dilations $\left\{\delta_{t}\right\}_{t>0}$. Then the correct family of non-Euclidean balls $B_{\mathcal{N}_{N r}}(x, \rho)$ is essentially dictated by translation- and dilation-invariance, starting with a more or less arbitrary harmless unit ball $B_{\mathcal{N}_{N r}}$ (identity, 1). Recall that the fundamental solution for $\widehat{\mathcal{L}}=\sum_{1}^{N} Y_{j}^{2}$ on $\mathcal{N}_{N r}$ is given by a kernel $K(x)$ homogeneous with respect to the $\delta_{t}$. Estimates that capture the size and smoothness of $K(x)$ may be phrased entirely in terms of the non-Euclidean balls $B_{\mathcal{N}_{N r}}(x, \rho)$. In fact, the basic estimates are as follows:

$$
\begin{aligned}
& \left|Y_{j_{1}} Y_{j_{2}} \cdots Y_{j_{m}} K(x)\right| \leq \frac{C_{m} \rho^{2-m}}{\operatorname{vol} B_{\mathcal{N}_{N r}}(0, \rho)} \\
& \quad \text { for } x \in B_{\mathcal{N}_{N r}}(0, \rho) \backslash B_{\mathcal{N}_{N r}}\left(0, \frac{\rho}{2}\right) \text { and } m \geq 0 .
\end{aligned}
$$

Next we associate non-Euclidean balls to a general Hörmander operator. For simplicity, take $\mathcal{L}=\sum_{1}^{N} X_{j}^{2}$ as in our discussion of [80] by Rothschild and Stein. One definition of the balls $B_{\mathcal{L}}(x, \rho)$ involves a moving particle that starts at $x$ and travels along the integral curve of $X_{j_{1}}$ for time $t_{1}$. From its new position $x^{\prime}$ the particle then travels along the integral curve of $X_{j_{2}}$ for time $t_{2}$. Repeating the process finitely many times, we can move the particle from its initial position $x$ to a final position $y$ in a total time $t=t_{1}+\cdots+t_{m}$. The ball $B_{\mathcal{L}}(x, \rho)$ consists of all $y$ that can be reached in this way in time $t<\rho$. For instance, if $\mathcal{L}$ is elliptic, then $B_{\mathcal{L}}(x, \rho)$ is essentially the ordinary (Euclidean) ball about $x$ of radius $\rho$. If we take $\widehat{\mathcal{L}}=\sum_{1}^{N} Y_{j}^{2}$ on $\mathcal{N}_{N, r}$, then the balls $B_{\widehat{\mathcal{L}}}(x, \rho)$ behave naturally under translations and dilations; hence they are essentially the same as the $B_{\mathcal{N}_{N r}}(x, \rho)$ appearing in (1.6.10). Nagel, Wainger, and Stein analyzed the relations between $B_{\widehat{\mathcal{L}}}(x, \rho), B_{\widetilde{\mathcal{L}}}(x, \rho)$ and $B_{\mathcal{L}}(x, \rho)$ for an arbitrary Hörmander operator $\mathcal{L}$. (Here $\widetilde{\mathcal{L}}$ and $\widehat{\mathcal{L}}$ are as in our previous discussion of Rothschild and Stein.) This allowed them to integrate out the extra variables in the fundamental solution of $\widetilde{\mathcal{L}}$, to derive the following sharp estimates from (I.6.10). 
Theorem I.6.3. Suppose $X_{1} \cdots X_{N}$ and their repeated commutators span the tangent space. Also, suppose we are in dimension greater than 2 . Then the solution of $\left(\sum_{1}^{N} X_{j}^{2}\right) u=f$ is given by $u(x)=\int K(x, y) f(y) d y$ with

$$
\left|X_{j_{1}} \cdots X_{j_{m}} K(x, y)\right| \leq \frac{C_{m} \rho^{2-m}}{\left(\operatorname{vol} B_{\mathcal{L}}(y, \rho)\right)} \text { for } x \in B_{\mathcal{L}}(y, \rho) \backslash B_{\mathcal{L}}\left(y, \frac{\rho}{2}\right) \quad \text { and } m \geq 0 \text {. }
$$

Here the $X_{j_{t}}$ act either in the $x$-or the $y$-variable.

Let us return from Hörmander operators to the $\bar{\partial}$-problems on strongly pseudoconvex domains $D \subset \mathbb{C}^{n}$. Greiner and Stein derived sharp estimates for the Neumann Laplacian $\square w=\alpha$ in their book [87]. This problem is hard because two different families play an important role. On the one hand, the standard (Euclidean) balls arise here, because $\square$ is simply the Laplacian in the interior of $D$. On the other hand, non-Euclidean balls (as in Folland and Stein [73]) arise on $\partial D$, because they are adapted to the nonelliptic boundary conditions for $\square$. Thus, any understanding of $\square$ requires notions that are natural with respect to either family of balls. A key notion is that of an allowable vector field on $\bar{D}$. We say that a smooth vector field $X$ is allowable if its restriction to the boundary $\partial D$ lies in the span of the complex vector fields $L_{1} \cdots L_{n-1}, \bar{L}_{1} \cdots \bar{L}_{n-1}$. Here we have retained the notation of our earlier discussion of $\bar{\partial}$-problems. At an interior point, an allowable vector field may point in any direction, but at a boundary point it must be in the natural codimension-1 subspace of the tangent space of $\partial D$. Allowable vector fields are well suited to the Euclidean and the Heisenberg balls that control $\square$. The sharp estimates of Greiner and Stein are as follows.

Theorem I.6.4. Suppose $\square w=\alpha$ on a strictly pseudoconvex domain $D \subset \mathbb{C}^{n}$. If $\alpha$ belongs to the Sobolev space $L_{k}^{p}$, then $w$ belongs to $L_{k+1}^{p}(1<p<\infty)$. Moreover, if $X$ and $Y$ are allowable vector fields, then $X Y w$ belongs to $L_{k}^{p}$. Also, Lw belongs to $L_{k+1}^{p}$ if $\bar{L}$ is a smooth complex vector field of type $(0,1)$. Similarly, if $\alpha$ belongs to the Lipschitz space $\operatorname{Lip}(\beta)(0<\beta<1)$, then the gradient of $w$ belongs to $\operatorname{Lip}(\beta)$ as well. Also the gradient of $\bar{L} w$ belongs to $\operatorname{Lip}(\beta)$ if $L$ is a smooth complex field of type $(0,1)$; and $X Y w$ belongs to $\operatorname{Lip}(\beta)$ for $X$ and $Y$ allowable vector fields.

These results for allowable vector fields were new even for $L^{2}$. We sketch the proof.

Suppose $\square w=\alpha$. Ignoring the boundary conditions for a moment, we have $\Delta w=\alpha$ in $D$, so

$$
w=G \alpha+\text { P.I. }(\widetilde{w}),
$$

where $\widetilde{w}$ is defined on $\partial D$, and $G$ and P.I. denote the standard Green's operator and Poisson integral, respectively. The trouble with (I.6.11) is that we know nothing about $\widetilde{w}$ so far. The next step is to bring in the boundary condition for $\square w=\alpha$. According to Calderón's work on general boundary-value problems, (I.6.11) satisfies the $\bar{\partial}$-Neumann boundary conditions if and only if

$$
A \widetilde{w}=\left.\{B(G \alpha)\}\right|_{\partial D}
$$

for a certain operator $B$ on $D$ and a certain pseudodifferential operator $A$ on $\partial D$. Both $A$ and $B$ can be determined explicitly from routine computation.

Greiner and Stein [87] derive sharp regularity theorems for the pseudodifferential equation $A \widetilde{w}=g$ and then apply those results to (‥6.12) in order to understand $\widetilde{w}$ 
in terms of $\alpha$. Once they know sharp regularity theorems for $\widetilde{w}$, formula (I.6.11) gives the behavior of $w$.

Let us sketch how Greiner and Stein analyzed $A \widetilde{w}=g$. This is really a system of $n$ pseudodifferential equations for $n$ unknown functions $\left(n=\operatorname{dim} \mathbb{C}^{n}\right)$. In a suitable frame, one component of the system decouples from the rest of the problem (modulo negligible errors) and leads to a trivial (elliptic) pseudodifferential equation. The nontrivial part of the problem is a first-order system of $(n-1)$ pseudodifferential operators for $(n-1)$ unknowns, which we write as

$$
\square_{+} w^{\#}=\alpha^{\#} .
$$

Here $\alpha^{\#}$ consists of the nontrivial components of $\left.\{B(G \alpha)\}\right|_{\partial D}, w^{\#}$ is the unknown, and $\square_{+}$may be computed explicitly.

Greiner and Stein reduce (I.6.13) to the study of the Kohn Laplacian $\square_{b}$. In fact, they produce a matrix $\square_{-}$of first-order pseudodifferential operators similar to $\square_{+}$, and then show that $\square_{-} \square_{+}=\square_{b}$ modulo negligible errors 4 Applying $\square_{-}$ to $(1.6 .13)$ yields

$$
\square_{b} w^{\#}=\square_{-} \alpha^{\#}+\text { negligible. }
$$

From Folland and Stein [73] one knows an explicit integral operator $K$ that inverts $\square_{b}$ modulo negligible errors. Therefore,

$$
w^{\#}=K \square_{-} \alpha^{\#}+\text { negligible. }
$$

Equations (I.6.11) and (1.6.15) express $w$ in terms of $\alpha$ as a composition of various explicit operators, including the Poisson integral, restriction to the boundary, $\square_{-}$, $K, G$. Because the basic notion of allowable vector fields is well behaved with respect to both the natural families of balls for $\square w=\alpha$, one can follow the effect of each of these very different operators on the relevant function spaces without losing information. To carry this out is a big job. We refer the reader to [87] for the rest of the story.

There have been important developments in the Stein program for several complex variables. In particular, we refer the reader to section II.5 in Part I for a discussion of singular Radon transforms, and to Nagel, Rosay, Stein, and Wainger [143], Chang, Nagel, and Stein [142], and [Mc, [Ch4, and [FK1] for the solution of the $\bar{\partial}$-problems on weakly pseudoconvex domains of finite type in $\mathbb{C}^{2}$.

Particularly in several complex variables are we able to see in retrospect the fundamental interconnections among classical analysis, representation theory, and partial differential equations, which Stein was the first to perceive.

\section{I.7. Conclusions}

Although the above discussion omits a significant part of Stein's work up to 1991, we hope it conveys an idea of its breadth, power, beauty, and originality. In Part II we discuss some of Stein's more recent work and comment on the major impact of his ideas on subjects ranging from evolution partial differential equations to analytic number theory.

\footnotetext{
${ }^{4}$ This procedure requires significant changes in two complex variables, since then $\square_{b}$ isn't invertible.
} 


\section{Part II. Recent advances and future directions}

Eli Stein was a broad mathematician, who made stunning discoveries in several different areas of analysis. In this chapter we discuss some of his work and its lasting impact on several fields, such as several complex variables, the theory of singular averages and Radon transforms, and discrete harmonic analysis. Eli's vision transformed and expanded these fields, and he identified new directions of research that have motivated much of the work in harmonic analysis over the last decades.

The sections in this chapter are contributed by some of Eli's students and collaborators. We were all very fortunate to have had Eli as a mentor at some point in our careers.

\section{II.1. Continuous AND Discrete RADON TRAnsforms}

\section{contributed by A. Ionescu, A. Magyar, and S. Wainger}

The analysis of averages of functions over surfaces has played a major role in Eli's work. Such averages arise naturally in geometric problems, for example in the analysis of the $\bar{\partial}$ operator (see section $\amalg$ II.5) or in the analysis of Poisson integrals on symmetric spaces. Eli recognized the significance of understanding these averages as part of a unifying theory connecting Fourier analysis and geometry of surfaces in Euclidean spaces.

Over more than four decades Eli and his collaborators developed powerful tools to analyze averages over surfaces systematically and efficiently. These tools later found unexpected applications in many seemingly unrelated problems, such as pointwise ergodic theorems (as we discuss in subsection [1.1.2), or analysis of solutions of semilinear evolution equations using Strichartz estimates.

To illustrate some of the main ideas, we consider the simplest case of onedimensional Radon transforms. Assume $\gamma: \mathbb{R} \rightarrow \mathbb{R}^{n}$ is a suitable function (say polynomial) satisfying $\gamma(0)=0$, and define the maximal average

$$
\mathcal{M} f(x):=\sup _{h>0} \frac{1}{2 h} \int_{-h}^{h}|f(x-\gamma(t))| d t
$$

and the singular integral operator

$$
\mathcal{H} f(x):=\int_{\mathbb{R}} f(x-\gamma(t)) K(t) d t,
$$

where $K$ is a Calderón-Zygmund kernel.

The systematic analysis of such averages was initiated in the early 1970s by Nagel, Rivière, Stein, and Wainger in a sequence of papers [56], [NRW1, NRW2, NRW3, [84], [86]. The main conclusions of these papers can be summarized as follows:

Theorem II.1.1. If $\gamma=\left(\gamma_{1}, \ldots, \gamma_{n}\right)$ is a polynomial curve, then the maximal operator $\mathcal{M}$ and the singular integral operator $\mathcal{H}$ extend to bounded operators on $L^{p}\left(\mathbb{R}^{n}\right)$,

$$
\|\mathcal{M} f\|_{L^{p}} \lesssim_{p}\|f\|_{L^{p}}, \quad\|\mathcal{H} f\|_{L^{p}} \lesssim_{p}\|f\|_{L^{p}}, \quad p \in(1, \infty)
$$

The proof of this theorem relied on many of the ideas in Eli's earlier work, such as interpolation of analytic families of operators, the role of curvature in estimating 
oscillatory integrals, and the connection between maximal functions and square functions.

Theorem I.1.1 can be extended to translation-invariant averages over more general smooth curves, or higher-dimensional surfaces. However, these extensions are not sufficient for many natural applications. What is really needed is a more robust theory, which allows for variable coefficient averages, over surfaces $\gamma(x, t)$ that depend on $x$.

Averages over moving surfaces that depend on the basepoint are much more subtle because the resulting operators cannot be described easily in terms of the Fourier transform. The analysis of this type of averages was initiated by Nagel, Stein, and Wainger [97], where the first $L^{2}$ theorems were proved. The key new idea was to use dyadic decompositions and exploit almost orthogonality using the Cotlar-Stein lemma (see Theorem I.4.1).

Over the next two decades Eli (and many others, including Christ who made important contributions to the theory in Ch1]) devoted a significant amount of effort to understanding averages over variable surfaces in various situations motivated by geometric problems. This required a refined and sophisticated framework that had to be able to incorporate both the geometric information of the moving surfaces $M_{x}$, described in terms of Lie algebras of vector fields, and the analytical components of the proof, such as almost orthogonality and interpolation. A definitive result of this effort, including $L^{p}$ estimates on both maximal and singular operators, at a suitable level of generality, was finally proved in 1999 by Christ, Nagel, Stein, and Wainger [182], using many of the earlier developments.

More recently, the theory of singular and maximal Radon transforms has been extended to the multiparameter setting; see section 1 I.8 below for a longer discussion.

II.1.1. Discrete operators on $\mathbb{Z}^{n}$. Discrete averages, both of the maximal and singular type, have only been considered more recently. Assume $P: \mathbb{Z} \rightarrow \mathbb{Z}$ is a polynomial map satisfying $P(0)=0$, and define the natural analogues of the operators in (II.1.1)- (II.1.2),

$$
\mathcal{M} f(x):=\sup _{N \geq 1} \frac{1}{2 N+1} \sum_{|n| \leq N}|f(x-P(n))|, \quad \mathcal{H} f(x):=\sum_{n \neq 0} f(x-P(n)) K(n),
$$

where $K$ is a Calderón-Zygmund kernel as before.

Bourgain [Bo4,Bo5, Bo7] initiated the study of discrete maximal averages, motivated by open problems in ergodic theory. The following theorem summarizes his results:

Theorem II.1.2. The maximal operator $\mathcal{M}$ is bounded on $L^{p}(\mathbb{Z})$ for any $p \in$ $(1, \infty]$. As a consequence, if $(X, \mathfrak{B}, \mu)$ is a Borel probability space and $T: X \rightarrow X$ is a measure-preserving invertible transformation, then the averages

$$
\mathcal{A}_{N} f(x):=\frac{1}{2 N+1} \sum_{|n| \leq N} f\left(T^{P(n)} x\right), \quad f \in L^{p}(X), p>1,
$$

converge pointwise and in $L^{p}$ as $N \rightarrow \infty$.

The techniques introduced by Bourgain in these papers have played a central role in the development of the entire subject. The main issue is to prove $L^{p}$ boundedness 
of the maximal function, from which the pointwise ergodic theorem follows by transference, using an additional limiting argument. We will discuss some of the main ideas below.

Slightly later, the study of discrete singular Radon transforms was initiated by Stein and Wainger [150]. Theorem II.1.3, which combines the results in [150] and IW], was proved by Ionescu, Stein, and Wainger.

Theorem II.1.3. The discrete singular integral operator $\mathcal{H}$ defined above extends to a bounded operator on $L^{p}(\mathbb{Z})$,

$$
\|\mathcal{H} f\|_{L^{p}} \lesssim p\|f\|_{L^{p}}, \quad p \in(1, \infty) .
$$

Naively, one could think of Theorems \I.1.2 and \.1.3 as direct extensions of Theorem II.1.1, where continuous averages are replaced by averages over integers. However, this is the case only for linear polynomials $P(n)=a n+b$. For higherdegree polynomials the discrete averages are a lot more sparse, because the polynomials select only a small number of values to be used in the average, and there are arithmetic issues to be understood.

To illustrate the method, consider averages of the form

$$
T_{k} f(x):=\sum_{n \in \mathbb{Z}} f(x-P(n)) \cdot 2^{-k} \varphi\left(n / 2^{k}\right),
$$

where $k \gg 1$ and $\varphi$ is a smooth function supported in the interval $[-1,1]$. One should think of $\varphi$ as having integral $\approx 1$ in the case of the maximal function, and having integral $=0$ in the singular integral case. We start by taking the Fourier transform, thus

$$
\widehat{T_{k} f}(\xi)=\widehat{f}(\xi) m_{k}(\xi), \quad m_{k}(\xi):=\sum_{n \in \mathbb{Z}} e^{-2 \pi i P(n) \xi} \cdot 2^{-k} \varphi\left(2^{-k} n\right),
$$

where $\xi \in[0,1]$. Then we would like to use the circle method: for frequencies $\xi$ in suitably defined minor arcs, we have $\left|m_{k}(\xi)\right| \lesssim 2^{-\delta k}, \delta>0$, using Weyl's lemma. The main contribution is therefore coming from major arcs, so one can think that

$$
m_{k}(\xi) \approx \sum_{a, q} S(a / q) \Phi_{k}(\xi-a / q)
$$

up to acceptable errors. Here the sum is taken over irreducible fractions $a / q$ with small denominator $q \leq 2^{\delta k}, S(a / q)$ are Gauss sums, and $\Phi_{k}$ are suitably localized multipliers that can be linked to the continuous Radon transforms.

After these reductions, it remains to consider operators defined by multipliers $m_{k}$ as in (II.1.8). The basic scheme involves three main ingredients:

(1) Bounds on the decay of the Gauss sums and the localization around the fractions $a / q$. The arithmetic information needed here is essentially at the level of the information needed to solve the Waring problem; in particular the Gauss sums $S(a / q)$ decay like $q^{-\delta}$.

(2) Good understanding of the multipliers $\Phi_{k}$. These multipliers come from the continuous Radon transforms, and their mapping properties are already well understood as part of the analysis of translation-invariant continuous Radon transforms (Theorem II.1.1).

(3) An orthogonality argument to sum over the fractions $a / q$ with small denominators. The issue here is that the $q^{-\delta}$ decay of the factors $S(a / q)$ is 
too slow to allow a simple summation, particularly when the exponent $p$ is far from 2. This is the most subtle part of the argument.

These ideas have turned out to be very flexible and robust, at least in the case of translation-invariant operators. As a result, they have been extended in many directions, by many authors. We mention just a few of these extensions here, mostly connected to Eli's work:

- Discrete spherical maximal averages of the form

$$
A(f)(x):=\sup _{\lambda \in[0, \infty)} \frac{1}{N_{d}(r)} \sum_{|n|=r}|f(x-n)|,
$$

where $f: \mathbb{Z}^{d} \rightarrow \mathbb{C}$ and $N(r)$ denotes the number of points in $n \in \mathbb{Z}$ with $|n|=r$, have been considered by Magyar, Stein, and Wainger $\mathrm{Mg}$, [192], who proved an optimal result in [192]: the maximal operator $A$ is bounded on $L^{p}\left(\mathbb{Z}^{d}\right)$ if and only if $d \geq 5$ and $p>d /(d-2)$ or $d \leq 4$ and $p=\infty$. See also [Io2], [Hg], and [KLM] for later extensions.

We notice that the acceptable range of exponents is more restricted than in the case of the continuous spherical maximal function, where the sharp range is $p>d /(d-1)$, as proved by Stein [83] in dimensions $d \geq 3$ and Bourgain Bo1] in dimension $d=2$. This is due to arithmetic obstructions, namely the fact that the function $N_{d}(r)$ is irregular in dimensions $d \leq 4$, and the observation that the spheres are codimension- 2 objects in dimensions $d \geq 5$.

- Discrete fractional integral operators of the form

$$
I_{\lambda}(f)(x):=\sum_{n \in \mathbb{Z} \backslash\{0\}} f(x-P(n)) n^{-\lambda}, \quad \lambda \in(0,1),
$$

have also been considered by Stein and Wainger [186], [193], Oberlin [Ob], and Pierce [Pi1], Pi2]. Such fractional integral operators are proved to be bounded from $L^{p}(\mathbb{Z})$ to $L^{q}(\mathbb{Z})$ for a suitable range of exponents that depends on $\lambda$. We note again that the range of exponents $(p, q)$ for which one can prove boundedness is more limited than the "natural" range suggested by continuous fractional integration; here this is due to unresolved problems in number theory such as Hypothesis $K^{*}$ of Hardy and Littlewood.

- Stronger theorems, including bounds on vector-valued operators and variation norms for discrete averages and truncated singular integrals were proved recently by Mirek, Stein, and Trojan in a sequence of papers [222], [226], Mi. These bounds improve on the maximal and singular integral averages described earlier in Theorems II.1.2 and I.1.3 and provide a unified framework for the entire theory.

II.1.2. Discrete averages on nilpotent groups. All the theorems described in the previous section are in the translation-invariant setting on the abelian group $\mathbb{Z}^{n}$. This plays a key role in all the proofs, since it allows one to use Fourier analysis techniques, in particular the powerful ideas in the circle method, to implement the scheme described above.

The situation is much less understood outside this framework, and only partial results are known. One could start by considering the next simplest case, namely group translation-invariant operators on nilpotent discrete groups, such as the Heisenberg group. Even in this case, there are many open problems. 
More precisely, assume $\mathbb{G}$ is a discrete nilpotent group and that $A: \mathbb{Z} \rightarrow \mathbb{G}$ is a polynomial sequence, and consider the following problems 5

\section{Conjecture II.1.4.}

(i) (Maximal Radon transforms) The maximal operator

$$
\mathcal{M} f(g)=\sup _{N \geq 0} \frac{1}{2 N+1} \sum_{|n| \leq N}\left|f\left(A^{-1}(n) \cdot g\right)\right|, \quad g \in \mathbb{G},
$$

is bounded on $L^{2}(\mathbb{G})$.

(ii) ( $L^{2}$ pointwise ergodic theorems) Assume $\mathbb{G}$ acts by measure-preserving transformations on a probability space $X, f \in L^{2}(X)$, and let

$$
A_{N} f(x)=\frac{1}{2 N+1} \sum_{|n| \leq N} f\left(A^{-1}(n) \cdot x\right), \quad x \in X .
$$

Then the sequence $A_{N} f$ converges almost everywhere in $X$ as $N \rightarrow \infty$.

(iii) (Singular Radon transforms) The singular Radon transform

$$
H f(g)=\sum_{n \in \mathbb{Z} \backslash\{0\}} K(n) f\left(A^{-1}(n) \cdot g\right), \quad g \in \mathbb{G},
$$

is bounded on $L^{2}(\mathbb{G})$, where $K: \mathbb{R} \rightarrow \mathbb{R}$ is a Calderón-Zygmund kernel.

In view of our earlier discussion, all these statements are known in the commutative case $\mathbb{G}=\mathbb{Z}^{d}$. However, very little is known in the case of noncommutative discrete nilpotent groups, even in the case of step 2 nilpotent groups. In fact, the pointwise ergodic problem in (ii) is a well-known conjecture in ergodic theory (see [BL]). The main difficulty is, of course, the absence of a good Fourier transform, at the level of precision of the circle method in the commutative setting.

The only partial results so far on such problems are those of Stein, Ionescu, Magyar, and Wainger [203], [204], and [MW]. These results are still far from the desired conclusions stated in Conjecture II.1.4 as they depend, essentially, on making favorable assumptions on the position of the polynomial sequence relative to the center of the group. A lot remains to be understood in the case of discrete Radon transforms over variable surfaces, even in the apparently simple case of translation-invariant Radon transforms relative to nilpotent groups.

In retrospect, it is clear that Eli's ability to identify interesting questions, place these questions in the right framework, and investigate these questions systematically, over decades, played a key role in development of the theory of Radon transforms.

\section{II.2. Pseudoconvexity And the CAuchy-Szegő Projection}

\section{contributed by L. Lanzani}

I met Eli for the first time when I was a graduate student at a summer school in Venice. Soon after the completion of my $\mathrm{PhD}$, we began what turned out to be a life-long collaboration which quickly grew into a close and, for me, deeply formative friendship.

The main focus of our joint work was on complex analysis, specifically on singular integral operators whose kernel is a holomorphic (analytic) function of the output

\footnotetext{
${ }^{5}$ We state this conjecture in the simplest case, but one can also consider similar problems for $L^{q}$ functions, $q>1$, or for multidimensional polynomial sequences $A: \mathbb{Z}^{k} \rightarrow \mathbb{G}, k \geq 1$.
} 
variable, such as the Cauchy integral or the Bergman and Cauchy-Szegö projections for, respectively, the holomorphic Bergman and Hardy spaces associated with a bounded domain $D$ in complex Euclidean space $\mathbb{C}^{n}, n \geq 1$.

We began with the one-complex-variable version of these operators (the planar case [197]) and soon after turned to the higher-dimensional setting $\left(D \Subset \mathbb{C}^{n}, n \geq 2\right)$. As is well known, for general domains $D$ in $\mathbb{C}^{n}, n \geq 2$, it is not natural to look for analogues of the Cauchy integral formula. Unless $D$ is a domain of holomorphy, there may be a strictly larger domain $D^{\prime}$ such that every analytic function on $D$ extends analytically to $D^{\prime}$. We will therefore restrict ourselves to the case when $D$ is a domain of holomorphy $\mathrm{Ra}$.

While every planar domain is a domain of holomorphy, in complex dimension $n=2$ and higher this is no longer the case: very simple examples were discovered by Hartogs already in 1906. It follows that holomorphic Cauchy-type kernels associated to $D \Subset \mathbb{C}^{n}$ with $n \geq 2$ must be domain specific. The study of existence and the construction of such kernels has been the object of intense scrutiny starting from the 1950s with the work of J. Leray: it consists of a fascinating blend of algebraic, analytic, and geometric constructs collectively known as the CauchyFantappiè theory, a prime example of which is Eli's seminal work with N. Kerzman from 1978, [89].

All the existing constructions of Cauchy-Fantappiè kernels require that the domain of holomorphy be in fact pseudoconvex [He], $\mathrm{Ho}$, and this, in turn, leads to restrictions on the domain's regularity: the boundary of $D$ must have some smoothness. Not surprisingly, all the earlier results were stated for smooth domains and the proofs relied on techniques 6 that are not applicable if $D$ lacks smoothness. About 30 years later, a surge of interest for singular integral operators in a variety of nonsmooth settings (see, e.g., [Ca1, Ch5], CMM], Da], [NTV], To]) led us to re-examine these problems from the following point of view: To what extent are the existence and key features of the aforementioned operators reliant upon the boundary regularity and (natural to this context) upon the amount of convexity of the ambient domain $D$ ?

In what follows I will highlight some of the work that appeared or will appear in [219], [221], [228], and [234].

II.2.1. The Cauchy integral in $\mathbb{C}^{n}$. The main result in [219] shows that the Cauchy-Leray integral $\mathcal{C}$ is a well-defined and bounded operator: $L^{p}(b D, \mu) \rightarrow$ $L^{p}(b D, \mu)$ for $1<p<\infty$ if the ambient domain $D \Subset \mathbb{C}^{n}$ is

(i) strongly $\mathbb{C}$-linearly convex, and

(ii) of class $C^{1,1}$.

Here $\mu$ belongs to a family of boundary measures that includes the induced Lebesgue measure $\Sigma$, the Leray-Levi measure $\lambda$, and Fefferman's measure [Fe5]. In the proof one has to, first, verify that the Cauchy-Leray kernel, which requires two derivatives of the defining function of $D$ and is therefore defined only a.e. in $\mathbb{C}^{n}$, is meaningful when integrated along the topological boundary of $D$ (a zero-measure subset of $\mathbb{C}^{n}$ ). Having established existence, the proof of $L^{p}$-regularity relies on the application of a $T(1)$-theorem technique for a space of homogeneous type fitted to the geometry and regularity of $D$.

\footnotetext{
${ }^{6}$ The techniques involved osculation by model domain (typically the Siegel upper half-space) with adequate control on the error, which happens only if $D$ is of class $C^{k}$ with $k \geq 3$.
} 
In [228] we construct two simple examples that establish the optimality of the assumptions (i) and (ii). Both examples are ellipsoids: In the first instance, the domain is smooth, strongly pseudoconvex, and strictly convex, but it is not strongly $\mathbb{C}$-linearly convex. The second example is strongly $\mathbb{C}$-linearly convex but is only of class $C^{1, \alpha}$ for $0<\alpha<1$ (and no better). In both cases the associated Cauchy-Leray integral is well defined on a dense subset of $L^{p}(b D, \mu)$ but does not extend to a bounded operator for any $1<p<\infty$.

II.2.2. Applications to the Cauchy-Szegő projection. Strong $\mathbb{C}$-linear convexity implies strong pseudoconvexity whenever the domain enjoys enough regularity for the latter to be meaningful. In a subsequent project [221], we applied the techniques developed in [219] to study $L^{p}$-regularity of the Cauchy-Szegö projection $\mathcal{S}$ associated to a strongly Levi-pseudoconvex domain $D \Subset \mathbb{C}^{n}$ with minimal boundary regularity, namely the class $C^{2}$, leading to the conclusion that for any such domain, $L^{p}(b D, \mu)$-boundedness of $\mathcal{S}$ holds in the full range $1<p<\infty$, with the measure $\mu$ as above.

The point of departure is a paradigm discovered by Stein and Kerzman [89] that relates $\mathcal{S}$ to a certain Cauchy-Fantappiè integra 7 : if the ambient domain $D$ is sufficiently smooth (class $C^{3}$ or better), a cancellation of singularity occurs that gives compactness of $\mathcal{C}^{*}-\mathcal{C}$ on $L^{p}(b D, \Sigma)$ leading to the Kerzman-Stein equation

$$
\mathcal{S}=\mathcal{C}\left[I-\left(\mathcal{C}^{*}-\mathcal{C}\right)\right]^{-1}
$$

Here $I$ is the identity operator on $L^{2}(b D, \Sigma)$. Thus $L^{p}$-regularity of $\mathcal{S}$ is a consequence of the corresponding result for $\mathcal{C}$.

If $D$ is only of class $C^{2}$, this argument is no longer applicable because $\mathcal{C}^{*}-\mathcal{C}$ in general fails to be compact [BL]. To get around this obstruction, one works with a family of holomorphic Cauchy-Fantappiè integrals $\left\{\mathcal{C}_{\epsilon}\right\}_{\epsilon}$ whose kernels are constructed via a first-order perturbation of the Cauchy-Leray kernel; such operators are bounded in $L^{p}(b D, \mu)$ for any $\mu$ in the aforementioned family of boundary measures by the T(1)-theorem technique of [219].

On the other hand, in defining the Cauchy-Szegö projection, one has to specify the measure that arises in the notion of orthogonality that is being used. Correspondingly, we now have a family of projections $\left\{\mathcal{S}_{\mu}\right\}_{\mu}$, but these, in our general setting, are not directly related to one another. It turns out that one member in our collection of measures, namely the Leray-Levi measure $\lambda$, has a unique, mitigating effect that leads to a new smallness argument for the difference $\mathcal{C}_{\epsilon}^{\dagger}-\mathcal{C}_{\epsilon}$ that occurs when the adjoint $\mathcal{C}_{\epsilon}^{\dagger}$ is computed with respect to $\lambda$. While the $\left\{\mathcal{C}_{\epsilon}\right\}_{\epsilon}$ do not approximate $\mathcal{S}_{\lambda}$ (in fact the norms of the $\mathcal{C}_{\epsilon}$ are in general unbounded as $\epsilon \rightarrow 0$ ), for each fixed $1<p<2$ there is $\epsilon=\epsilon(p)$ such that $\mathcal{C}_{\epsilon}^{\dagger}-\mathcal{C}_{\epsilon}$ splits as the sum $\mathcal{B}_{\epsilon}+\mathcal{A}_{\epsilon}$, where $\mathcal{B}_{\epsilon}: L^{p}(b D, \lambda) \rightarrow C(b D)$, and $\left\|\mathcal{A}_{\epsilon}\right\|_{L^{p} \rightarrow L^{p}} \leq \epsilon$, giving us the following new variant of the Kerzman-Stein equation: $\mathcal{S}_{\lambda}=\left(\mathcal{S}_{\lambda} \mathcal{B}_{\epsilon}+\mathcal{C}_{\epsilon}\right)\left(I-\mathcal{A}_{\epsilon}\right)^{-1}$. When the adjoint $\mathcal{C}_{\epsilon}^{*}$ is computed with respect to $\mu \neq \lambda$, there is no direct way to show smallness for $\mathcal{C}_{\epsilon}^{*}-\mathcal{C}_{\epsilon}$, but it is still possible to recover such smallness indirectly, from the corresponding result for $\mathcal{C}_{\epsilon}^{\dagger}-\mathcal{C}_{\epsilon}$.

\footnotetext{
${ }^{7}$ which we keep denoting $\mathcal{C}$, with slight abuse of notation.
} 
My final collaboration with Eli concerned the investigation of optimality of the assumption of strong pseudoconvexity in the proof of $L^{p}$-regularity of the CauchySzegö projection; studying this problem led us to answer a long-standing open question concerning $L^{p}$-irregularity of the Cauchy-Szegö projection for the DiederichFornass worm domains [Ba]. We defer to [234] for a description of the problem and for the statement of the main result; the complete proof will appear elsewhere.

\section{II.3. Dimension-FREE ESTIMATES AND VARIATION NORMS}

\section{contributed by M. Mirek}

The problems undertaken in my joint work with Eli are motivated by some results from classical harmonic analysis and ergodic theory. On one hand, we were concerned with developing dimension-free estimates for continuous and discrete maximal functions corresponding to the Hardy-Littlewood averaging operators defined over convex bounded symmetric bodies, which are probably the simplest instances of maximal Radon operators. On the other hand, we also studied discrete operators of Radon type modeled on polynomial mappings.

II.3.1. Pointwise convergence problems in harmonic analysis and ergodic theory. Let $(X, \mathcal{B}, \mu)$ be a $\sigma$-finite measure space, and let $\left(T_{t} f: t>0\right)$ be a family of linear operators acting on $L^{1}(X, \mu)+L^{\infty}(X, \mu)$.

The classical strategy for handling pointwise convergence of a family of operators $T_{t} f(x)$ (as $t \rightarrow 0$ or $t \rightarrow \infty$ ) is based on a two-step procedure:

(i) The first step requires $L^{p}(X, \mu)$ boundedness, (when $p \in(1, \infty)$ ), or weak type $(1,1)$ bound (when $p=1$ ) for the corresponding maximal function $\sup _{t>0}\left|T_{t} f(x)\right|$. This ensures that the set of all functions $L^{p}(X, \mu)$, such that the limits

$$
\lim _{t \rightarrow 0} T_{t}(x) \quad \text { or } \quad \lim _{t \rightarrow \infty} T_{t}(x)
$$

exist $\mu$-almost everywhere on $X$, is closed in $L^{p}(X, \mu)$.

(ii) In the second step the task is reduced to finding a dense class of functions in $L^{p}(X, \mu)$ for which we have pointwise convergence.

These two steps guarantee that (II.3.1) holds for all $f \in L^{p}(X, \mu)$. Although in questions in harmonic analysis there are many natural dense subspaces which could be used to establish pointwise convergence, in discrete or in ergodic theoretical questions this may not be the case. We shall see more examples later.

In Bo4, Bo5, Bo7 Bourgain proposed two approaches to study (II.3.1). The first approach is based on $L^{2}$-boundedness of the so-called oscillation inequalities. The second approach, more refined, involves $r$-variational estimates. We focus our attention on the $r$-variational approach, which will allow us to establish pointwise convergence in one step. More precisely, let $\left(\mathfrak{a}_{t}(x): t>0\right)$ be a family of complexvalued functions 8 Recall that for any $r \geq 1$, the $r$-variational seminorm $V_{r}$ is defined by

$$
V_{r}\left(\mathfrak{a}_{t}(x): t \in Z\right)=\sup _{J \in \mathbb{N}} \sup _{\substack{t_{0}<\cdots<t_{J} \\ t_{j} \in Z}}\left(\sum_{j=0}^{J-1}\left|\mathfrak{a}_{t_{j+1}}(x)-\mathfrak{a}_{t_{j}}(x)\right|^{r}\right)^{1 / r},
$$

\footnotetext{
${ }^{8}$ To avoid problems with measurability, we always assume that $(0, \infty) \ni t \mapsto \mathfrak{a}_{t}(x)$ is continuous for $\mu$-almost every $x \in X$.
} 
where the supremum is taken over all finite increasing sequences in $Z \subseteq(0, \infty)$.

If for some $r \in[1, \infty)$ and $p \in[1, \infty)$ there is a constant $C_{p, r}>0$ such that we have

$$
\left\|V_{r}\left(T_{t} f: t>0\right)\right\|_{L^{p}} \leq C_{p, r}\|f\|_{L^{p}} \quad \text { for all } f \in L^{p}(X, \mu),
$$

then inequality (II.3.3) implies that $V_{r}\left(T_{t} f(x): t>0\right)$ is finite $\mu$-almost everywhere on $X$, which in turn implies that the family $\left(T_{t} f(x): t>0\right)$ is Cauchy and (II.3.1) holds. The most important benefit of inequality (II.3.3) is that it immediately implies pointwise convergence without establishing it on a dense class of functions in $L^{p}(X, \mu)$, which in many cases may be a very challenging task. However, the $r$-variational estimates are harder to obtain than the maximal ones. Namely, for any $r \geq 1$ and $t_{0}>0$, we have the pointwise bound

$$
\sup _{t>0}\left|T_{t} f(x)\right| \leq V_{r}\left(T_{t} f(x): t>0\right)+\left|T_{t_{0}} f(x)\right| .
$$

The $r$-variation is closely related to the $\lambda$-jump counting function that is defined for any $\lambda>0$ by

$$
N_{\lambda}\left(\mathfrak{a}_{t}(x): t \in Z\right)=\sup \left\{J \in \mathbb{N}: \exists_{t_{0}<\cdots<t_{J}} \min _{\substack{t_{j} \in Z \\ 0 \leq j<J}}\left|\mathfrak{a}_{t_{j+1}}(x)-\mathfrak{a}_{t_{j}}(x)\right| \geq \lambda\right\}
$$

which is an even more fundamental object. It is easy to see that for every $r \geq 1$ we have

$$
\sup _{\lambda>0}\left\|\lambda N_{\lambda}\left(T_{t} f: t>0\right)^{1 / r}\right\|_{L^{p}} \leq\left\|V_{r}\left(T_{t} f: t>0\right)\right\|_{L^{p}}
$$

since for all $\lambda>0$ we have $\lambda N_{\lambda}\left(T_{t} f(x): t>0\right)^{1 / r} \leq V_{r}\left(T_{t} f(x): t>0\right)$ by (II.3.2) and (ㅍ.3.4).

The remarkable feature of the $\lambda$-jumps, observed by Bourgain Bo7, is that, in some sense, inequality (II.3.5) can be reversed. Namely, a priori uniform $\lambda$-jump estimates

$$
\sup _{\lambda>0}\left\|\lambda N_{\lambda}\left(T_{t} f: t>0\right)^{1 / \rho}\right\|_{L^{p}} \leq C_{p, \rho}\|f\|_{L^{p}} \quad \text { for all } f \in L^{p}(X, \mu),
$$

for all $p \in(1, \infty)$, and some $\rho \in[1, \infty]$, imply strong $r$-variational estimates in (II.3.3) for the same range of $p$ and for any $r \in(\rho, \infty]$. We refer to [JSW] and [222], [226] for more details and the references given therein. In other words, if one is concerned in establishing $r$-variational estimates (II.3.3) in a certain range $r \in(\rho, \infty]$, it suffices to understand the corresponding $\lambda$-jump estimates (II.3.6) at the endpoint for $r=\rho$. Therefore, the jump inequality (II.3.6) is thought of as an endpoint estimate for $r$-variations at $r=\rho$. Finally, we see that (II.3.6) gives us some quantitative information about the convergence in (II.3.1).

\section{II.3.2. Dimension-free estimates for $r$-variations and $\lambda$-jumps in the con-} tinuous and discrete case. In the 1980s dimension-free estimates for the HardyLittlewood maximal functions over symmetric convex bodies had begun to be studied and went through a period of considerable change and development [Bo2, Bo3, Cb, Mü], [111], [116]. We refer also to more recent results [Al], Bo9, [225], [227], [230], and the survey article [DGM] for a very careful exposition of the subject. In order to describe these results more precisely, we fix some notation and terminology. Let $G$ be a bounded, closed, and symmetric convex subset of $\mathbb{R}^{d}$ with nonempty interior, which will simply be called a symmetric convex body. We set 
$G_{t}=\left\{y \in \mathbb{R}^{d}: t^{-1} y \in G\right\}$ for every $t>0$, and for every $x \in \mathbb{R}^{d}$ and $f \in L_{\text {loc }}^{1}\left(\mathbb{R}^{d}\right)$ we define the Hardy-Littlewood averaging operator

$$
M_{t}^{G} f(x)=\frac{1}{\left|G_{t}\right|} \int_{G_{t}} f(x-y) \mathrm{d} y .
$$

We know from [Bo5, $\mathrm{Cb}$ that for every $p \in(3 / 2, \infty]$, there is $C_{p}>0$ independent of the dimension such that for every convex symmetric body $G \subset \mathbb{R}^{d}$ we have

$$
\left\|\sup _{t>0}\left|M_{t}^{G} f\right|\right\|_{L^{p}} \leq C_{p}\|f\|_{L^{p}} \quad \text { for every } f \in L^{p}\left(\mathbb{R}^{d}\right) .
$$

For the lacunary variant of $M_{t}^{G}$ the range of $p$ 's can be extended. Namely, for every $p \in(1, \infty]$, there is $C_{p}>0$ independent of $d \in \mathbb{N}$ such that for every convex symmetric body $G \subset \mathbb{R}^{d}$ we have

$$
\left\|\sup _{n \in \mathbb{Z}}\left|M_{2^{n}}^{G} f\right|\right\|_{L^{p}} \leq C_{p}\|f\|_{L^{p}} \quad \text { for every } f \in L^{p}\left(\mathbb{R}^{d}\right) .
$$

It is conjectured that the inequality in (II.3.8) holds for all $p \in(1, \infty]$ and for all convex symmetric bodies $G \subset \mathbb{R}^{d}$ with the implied constant independent of $d$. It is reasonable to believe that this is true, since it was verified for a large class of convex symmetric bodies. If $G=B^{q}$ for $q \in[1, \infty]$, where $B^{q}$ is a ball induced by a small $\ell^{q}$ norm in $\mathbb{R}^{d}$, i.e.,

$$
B^{q}=\left\{x \in \mathbb{R}^{d}:|x|_{q}=\left(\sum_{1 \leq k \leq d}\left|x_{k}\right|^{q}\right)^{1 / q} \leq 1\right\}
$$

and

$$
B^{\infty}=\left\{x \in \mathbb{R}^{d}:|x|_{\infty}=\max _{1 \leq k \leq d}\left|x_{k}\right| \leq 1\right\},
$$

then the inequality in (II.3.8) holds for all $p \in(1, \infty]$ with a constant $C_{p, q}>0$, which is independent of the dimension. The case $G=B^{q}$ for $q \in[1, \infty)$ was handled by Müller [Mü], and the case $G=B^{\infty}$ of cubes was recently handled by Bourgain [Bo9]. Aldaz [Al] showed that the weak type $(1,1)$ bounds for (II.3.7) with $G=B^{\infty}$ grow to infinity with the dimension.

Recently in [225] in collaboration with Bourgain, Stein and Wróbel we initiated systematic studies of dimension-free estimates for (II.3.7) in the context of $r$-variations. Not long afterwards, with Stein and Zorin-Kranich [229], [230], we extended the results from [225]. Our efforts from [225], [229], and [230] can be summarized as follows.

Theorem II.3.1 ([225], [230]). If $p \in(3 / 2,4)$, then there is a constant $C_{p}>0$ independent of the dimension $d \in \mathbb{N}$ such that for every symmetric convex body $G \subset \mathbb{R}^{d}$ we have

$$
\sup _{\lambda>0}\left\|\lambda N_{\lambda}\left(M_{t}^{G} f: t>0\right)^{1 / 2}\right\|_{L^{p}} \leq C_{p}\|f\|_{L^{p}} \quad \text { for all } f \in L^{p}\left(\mathbb{R}^{d}\right) .
$$

In particular, (II.3.9) implies that for every $p \in(3 / 2,4)$ and $r \in(2, \infty)$ there is $C_{p, r}>0$ independent of the dimension $d \in \mathbb{N}$ such that

$$
\left\|V_{r}\left(M_{t}^{G} f: t>0\right)\right\|_{L^{p}} \leq C_{p, r}\|f\|_{L^{p}} \quad \text { for all } f \in L^{p}\left(\mathbb{R}^{d}\right) .
$$

If $G=B^{q}$ is one of the balls defined above for some $q \in[1, \infty]$, then the estimates (II.3.9) and (II.3.10) remain true for all $p \in(1, \infty)$. Moreover, the range for the 
parameter $p$ in (II.3.9) can be improved if we consider only long jumps. Namely, for every $p \in(1, \infty)$, there is $C_{p}>0$ independent of the dimension $d \in \mathbb{N}$ such that

$$
\sup _{\lambda>0}\left\|\lambda N_{\lambda}\left(M_{2^{n}}^{G} f: n \in \mathbb{Z}\right)^{1 / 2}\right\|_{L^{p}} \leq C_{p}\|f\|_{L^{p}} \quad \text { for all } f \in L^{p}\left(\mathbb{R}^{d}\right) .
$$

In particular, (II.3.11) implies that for every $p \in(1, \infty)$ and $r \in(2, \infty)$ there is $C_{p, r}>0$ independent of the dimension $d \in \mathbb{N}$ such that

$$
\left\|V_{r}\left(M_{2^{n}}^{G} f: n \in \mathbb{Z}\right)\right\|_{L^{p}} \leq C_{p, r}\|f\|_{L^{p}} \quad \text { for all } f \in L^{p}\left(\mathbb{R}^{d}\right) .
$$

Inequalities (II.3.10) and (II.3.12) were proved in [225]. In [230] we extended the results from [225] to the endpoint $r=2$. Now in view of general relations between the jumps and $r$-variations (see (II.3.6) and the discussion below), inequalities (II.3.9) and (II.3.11) imply inequalities (II.3.10) and (II.3.12), respectively.

The proof of inequalities in Theorem II.3.1 consists of two parts. The first part, the estimates for long jumps (II.3.11) or long $r$-variations (II.3.12), can be reduced to the dimension-free jump or $r$-variational estimates for symmetric diffusion semigroups - in our proofs we specifically used the Poisson semigroup. The second part is based on the dimension-free estimates for short variations (a square function corresponding to two-variations that are taken over all dyadic blocks); this is the place where the constraint for $p \in(3 / 2,4)$ comes from. Our approach is based on certain bootstrap arguments, which were recently adjusted to abstract settings in [230].

The range of $p \in(1, \infty)$ and $r \in(2, \infty)$ in inequalities (II.3.12), and (II.3.10) with $G=B^{q}$ for some $q \in[1, \infty]$ is sharp. It is also conjectured that the same should be true for (II.3.10) with general bodies $G \subset \mathbb{R}^{d}$, but it remains open even for $r=\infty$, which corresponds to the maximal function; see (II.3.8).

At the same time as [227], [232], [233], in collaboration with Bourgain, Stein, and Wróbel we originated systematic studies of dimension-free phenomena in the discrete setting. For every $x \in \mathbb{Z}^{d}$ and $t>0$ and for every function $f \in \ell^{1}\left(\mathbb{Z}^{d}\right)$, we define the discrete Hardy-Littlewood averaging operator over $G_{t} \cap \mathbb{Z}^{d}$ by

$$
\mathcal{M}_{t}^{G} f(x)=\frac{1}{\left|G_{t} \cap \mathbb{Z}^{d}\right|} \sum_{y \in G_{t} \cap \mathbb{Z}^{d}} f(x-y) .
$$

So $\mathcal{M}_{t}^{G}$ is a discrete analogue of the integral Hardy-Littlewood averaging operator $M_{t}^{G}$ from (II.3.7).

Surprisingly, it turned out in [227] that the dimension-free estimates in the discrete case are not as broad as in the continuous setup. We were able to construct an example of a symmetric convex body in $\mathbb{Z}^{d}$ for which maximal estimates on $\ell^{p}\left(\mathbb{Z}^{d}\right)$ for every $p \in(1, \infty)$ involve constants which are unbounded as $d \rightarrow \infty$.

On the other hand, we proved in [227] some results with positive conclusions.

Theorem II.3.2. For every $p \in(3 / 2, \infty]$ there is a constant $C_{p}>0$ such that for every $d \in \mathbb{N}$, we have

$$
\left\|\sup _{t>0}\left|\mathcal{M}_{t}^{B^{\infty}} f\right|\right\|_{\ell^{p}} \leq C_{p}\|f\|_{\ell^{p}} \quad \text { for every } f \in \ell^{p}\left(\mathbb{Z}^{d}\right) .
$$

Moreover, if the supremum in (II.3.14) is restricted to the dyadic times (i.e., $t \in$ $\left\{2^{n}: n \in \mathbb{N}_{0}\right\}$ ), then (II.3.14) remains true for every $p \in(1, \infty]$.

In [227] we were also able to obtain analogues of $r$-variational estimates (II.3.10) and (II.3.12) for $\mathcal{M}_{t}^{B^{\infty}}$ with the same ranges for $p$ and $r$. In [230] we extended 
these $r$-variational estimates to the endpoint $r=2$, and we obtained analogues of jump inequalities (II.3.9) and (II.3.11) for $\mathcal{M}_{t}^{B^{\infty}}$ with the same ranges for $p$. The case of discrete Euclidean balls was considered in [232], [233]. Finally, it is worth mentioning that the operators from (II.3.7) and (II.3.13) have ergodic interpretations and some pointwise ergodic theorems hold.

\section{II.4. MUltiparAmeter SINGULAR INTEGRALS AND APPLICATIONS}

contributed by A. Nagel and F. Ricci

A major theme running throughout Stein's work in harmonic analysis and highlighted in his books [49], [57], [161] is the important role played by one-parameter structures in the underlying space. One only needs to note the focus on oneparameter families of dilations, balls, and averages, the study of singular integral operators homogeneous with respect to one-parameter groups of dilations, and the study of more general classes of operators invariant under such symmetries. On the other hand, results involving multiparameter structures also have a long history, going back at least to the Marcinkiewicz multiplier theorem and to the 1935 paper of Jessen, Marcinkiewicz, and Zygmund [JMZ on strong maximal functions. Stein was deeply interested in such questions as well, and our objective here is to describe some of Stein's work with his collaborators on multiparameter structures and their application to problems in nilpotent Lie groups and several complex variables.

II.4.1. Early work on the product theory. Stein's early work in this area dealt with classes of integral operators and maximal functions on Euclidean space invariant under multiparameter groups of dilations. The 1982 paper [109] with Robert Fefferman establishes $L^{2}$ - and $L^{p}$-boundedness for singular integral kernels on $\mathbb{R}^{n} \times \mathbb{R}^{m}$ satisfying size estimates and cancellation conditions appropriate to the two-parameter dilations given by

$$
D_{\delta_{1}, \delta_{2}}\left(x_{1}, \ldots, x_{n}, y_{1}, \ldots, y_{m}\right)=\left(\delta_{1} x_{1}, \ldots, \delta_{1} x_{n}, \delta_{2} y_{1}, \ldots, \delta_{2} y_{m}\right) .
$$

Such kernels are generalizations of the double Hilbert transform $f \rightarrow \mathrm{pv} \frac{1}{x y} * f$ on $\mathbb{R} \times \mathbb{R}$, but new arguments are needed to establish boundedness since the kernel need not be written as a product of a singular integral in $x$ times a singular integral in $y$.

The theme of multiparameter dilations appears again ten years later in Stein's paper [155] with Fulvio Ricci. In the intervening decade the approach to singular integrals had shifted, thanks to work of Stein himself, Christ, Duoandikoetxea, Rubio de Francia, and many others. There was now more emphasis on the interpretation of a singular integral kernel as an infinite sum of dyadically scaled bump functions (or measures) satisfying uniform but otherwise quite mild regularity conditions, and having appropriate cancellation. The paper [155] considers dyadic sums of dilates of a family of distributions $\left\{\mu^{(I)}: I \in \mathbb{Z}^{k}\right\}$ under a $k$-parameter family of dilations on $\mathbb{R}^{n}$ given by

$$
D_{\delta_{1}, \ldots, \delta_{k}}^{\Lambda}\left(x_{1}, \ldots, x_{n}\right)=\left(\delta_{1}^{\lambda_{1,1}} \cdots \delta_{k}^{\lambda_{1, k}} x_{1}, \ldots, \delta_{1}^{\lambda_{n, 1}} \cdots \delta_{k}^{\lambda_{n, k}} x_{n}\right) .
$$

Here $\Lambda=\left\{\lambda_{i, j}\right\}$ is a real $n \times k$ matrix. This generalizes the Zygmund dilations on $\mathbb{R}^{3}, D_{\delta_{1}, \delta_{2}}\left(x_{1}, x_{2}, x_{3}\right)=\left(\delta_{1} x_{1}, \delta_{2} x_{2}, \delta_{1} \delta_{2} x_{3}\right)$ already studied by Nagel and Wainger 
[NW]. Writing $2^{\Lambda I}=D_{2^{i_{1}}, \ldots, 2^{i_{k}}}^{\Lambda}$, Ricci and Stein study kernels

$$
K(\mathbf{x})=\sum_{I \in \mathbb{Z}^{k}} \operatorname{det}\left(2^{-\Lambda I}\right) \mu^{(I)}\left(2^{-\Lambda I} \mathbf{x}\right)
$$

They find natural cancellation and regularity conditions on the family $\left\{\mu^{(I)}: I \in\right.$ $\left.\mathbb{Z}^{n}\right\}$ so that convolution with $K$ is bounded on $L^{2}\left(\mathbb{R}^{n}\right)$ or $L^{p}\left(\mathbb{R}^{n}\right)$. In particular, such kernels can be supported on lower-dimensional sets.

In papers [109] and [155] the classes of kernels $K$ are explicitly invariant under multiparameter dilations, and the corresponding operators $\mathcal{K}$ act on functions by standard Euclidean convolution: $\mathcal{K} f(x)=\int K(x-y) f(y) d y$. The Euclidean product setting is the natural one for problems related to rectangular convergence of multiple Fourier series, strong maximal functions, etc. However, much of Stein's work on multiparameter problems was also motivated by questions related to operators arising in several complex variables or to operators generated by vector fields satisfying Hörmander's spanning hypothesis.

Already in the early 1980s Phong and Stein [107] had studied questions related to the compositions of two pseudodifferential operators, one satisfying standard isotropic estimates and the other satisfying nonisotropic estimates. Such operators arise, for example, in the parametrix for the $\bar{\partial}$-Neumann problem on strictly pseudoconvex domains in $\mathbb{C}^{n}$ and on domains of finite type in $\mathbb{C}^{2}$ (see [87], [142], and $[107])$.

An example from the paper [107] is the following. Let $\mathbb{H}^{1} \cong \mathbb{C}^{1} \times \mathbb{R}$ be the one-dimensional Heisenberg group with multiplication

$$
\left(z_{1}, t_{1}\right) \cdot\left(z_{2}, t_{2}\right)=\left(z_{1}+z_{2}, t_{1}+t_{2}+2 \operatorname{Im} z_{1} \overline{z_{2}}\right) .
$$

Let $K_{1}, K_{2}$ be two Calderón-Zygmund kernels on $\mathbb{C}^{1} \times \mathbb{R}$, the first homogeneous with respect to the standard isotropic dilations $K_{1}(\lambda z, \lambda t)=\lambda^{-a} K_{1}(z, t)$, and the second homogeneous with respect to the automorphic dilations $K_{2}\left(\lambda z, \lambda^{2} t\right)=$ $\lambda^{-b} K_{2}(z, t)$ on the Heisenberg group. The convolution $K(z, t)=K_{1} * K_{2}(z, t)$ (using either Euclidean convolution or convolution on the group $\mathbb{H}^{1}$ ) is no longer a Calderón-Zygmund kernel of either type, but Phong and Stein find necessary and sufficient conditions for the operator $\mathcal{K} f=K * f$ to be $L^{p}$ bounded or weak-type $1-1$.

Another example of a problem studied in [107] is that of $L^{p}$-boundedness and weak-type 1-1 of convolution by a pointwise product $K_{1}(z, t) K_{2}(z, t)$, with $K_{1}$ homogeneous of degree $-a$ relative to one type of dilations and $K_{2}$ homogeneous of degree $-b$ relative to the other. Both examples hint the presence of interesting classes of convolution kernels which are product type, but not Calderón-Zygmund. The results of this paper are one of the main motivations for several further investigations of multiparameter problems by Stein and his collaborators, and we describe four of these.

II.4.2. Multi-parameter structure on Heisenberg-type groups. In the papers [168] and [171] Müller, Ricci, and Stein obtained versions of the Marcinkiewicz multiplier theorem on nilpotent Lie groups of Heisenberg type. To illustrate, consider the Heisenberg group $\mathbb{H}^{1}$ with coordinates $(x, y, t)$. Then a basis for the Lie algebra is given by the vector fields $X=\partial_{x}+2 y \partial_{t}, Y=\partial_{y}-2 x \partial_{t}$, and $T=\partial_{t}$, and the sub-Laplacian is the second-order operator $\mathcal{L}=-\left(X^{2}+Y^{2}\right)$. The operators $\mathcal{L}$ and $T$ commute, and by the spectral theorem, if $m(\xi, \eta)$ is a bounded function, 
the operator $m(\mathcal{L}, T)$ is bounded on $L^{2}\left(\mathbb{H}^{1}\right)$. With respect to the automorphic dilations $\delta_{\lambda}(z, t)=\left(\lambda z, \lambda^{2} t\right)$, the operators $\mathcal{L}$ and $T$ are both of second order, and a one-parameter theory (see Mauceri $[\mathrm{Mu}]$ ) shows that if $m$ satisfies the differential inequalities

$$
\left|\partial_{\xi}^{\alpha} \partial_{\eta}^{\beta} m(\xi, \eta)\right| \leq C_{\alpha, \beta}(|\xi|+|\eta|)^{-\alpha-\beta},
$$

then $m(\mathcal{L}, T)$ is bounded on $L^{p}\left(\mathbb{H}^{1}\right), 1<p<\infty$. However this result does not cover many functions of $\mathcal{L}$ and $T$ which are of interest. For example, to show that the elliptic operator $\mathcal{L}-T^{2}$ is controlled on $L^{p}\left(\mathbb{H}^{1}\right)$ by the hypoelliptic operator $\mathcal{L}$, one needs to consider the operators $\mathcal{L}\left(\mathcal{L}-T^{2}\right)^{-1}$ and hence the function $m(\xi, \eta)=$ $\xi\left(\xi-\eta^{2}\right)^{-1}$ which does not satisfy (II.4.1). What is needed is a Marcinkiewicz-type theorem which allows one to establish $L^{p}$-boundedness for functions $m$ satisfying the weaker condition

$$
\left|\partial_{\xi}^{\alpha} \partial_{\eta}^{\beta} m(\xi, \eta)\right| \leq C_{\alpha, \beta}|\xi|^{-\alpha}|\eta|^{-\beta}
$$

when $\alpha+\beta \leq N$ for $N$ sufficiently large. To do this, one would like to independently scale the operators $\mathcal{L}$ and $T$, but the two-parameter family of dilations $D_{\lambda, \mu}(x, y, t)=(\lambda x, \lambda y, \mu t)$ is not an automorphism of $\mathbb{H}^{1}$. Nevertheless the paper [168] establishes $L^{p}$-boundedness of $m(\mathcal{L}, T)$ for $m$ satisfying (II.4.2). A sharp value for the minimum number $N$ of derivatives needed is established in [171].

The argument for $L^{p}$-boundedness proceeds as follows.

(1) Consider the group $G=\mathbb{H}^{1} \times \mathbb{R}$. The two-parameter family of dilations $D_{\lambda, \mu}(x, y, t ; u)=\left(\lambda x, \lambda y, \lambda^{2} t ; \mu u\right)$ is an automorphism of $G$. Let $\mathcal{L}^{\#}$ be the operator on $G$ given by $\mathcal{L}$ acting on the $(x, y, t)$-variables in $G$. Let $U=\partial_{u}$ act on the $u$-variable in $\mathbb{R}$. The operators $\mathcal{L}^{\#}$ and $U$ commute, and the work in [168] develops a multiple-parameter product theory which shows that operators $m\left(\mathcal{L}^{\#}, U\right)$ are bounded on $L^{p}(G)$ for $1<p<\infty$ if $m$ satisfies (II.4.2). The proof involves Littlewood-Paley estimates on $\mathbb{H}^{1}$ and on $\mathbb{R}$ along with a one-parameter theory on each factor.

(2) Define $\pi: G \rightarrow \mathbb{H}^{1}$ by setting $\pi(x, y, t ; s)=(x, y, t+s)$, and if $f \in L^{1}(G)$ put

$$
f^{b}(z, t)=\int_{-\infty}^{+\infty} f(z, t-u, u) d u .
$$

The operator $m\left(\mathcal{L}^{\#}, U\right)$ on $G$ is given by convolution on $G$ with a producttype kernel $K(z, t, u)$, and this operator is bounded in $L^{p}(G)$ for $1<p<\infty$. One then makes the following observations.

(i) The operator $m(\mathcal{L}, T)$ on $\mathbb{H}^{1}$ is given by convolution with the function $K^{b}$.

(ii) If $\mathcal{R}_{w, s, v} f(z, t)=f((z, t) \cdot(w, s+v))$, then $\mathcal{R}$ is a representation of the group $G$ on the space $L^{p}\left(\mathbb{H}^{1}\right)$.

(iii) Using the representation $\mathcal{R}$, the operator $m\left(\mathcal{L}^{\#}, U\right)$ on $G$ is transferred to the operator $m(\mathcal{L}, T)$ on $\mathbb{H}^{1}$, and so $m(\mathcal{L}, T)$ is bounded on $L^{p}\left(\mathbb{H}^{1}\right)$. One can also completely describe the singular integral kernels $K(z, t)$ corresponding to the operators $m(\mathcal{L}, T)$ above.

Theorem II.4.1. Suppose that a multiplier $m(\xi, \eta)$ satisfies the inequalities in equation (II.4.2) for all $\alpha, \beta$. Let $K(z, t)$ be the corresponding convolution kernel, and assume that $K$ is radial in the $z$-variable. Then 
(a) $K$ is smooth away from the set where $z=0$, and satisfies

$$
\left|\partial_{z}^{j} \partial_{t}^{k} K(z, t)\right| \leq C_{j, k}|z|^{-2-j}\left(|z|^{2}+|t|\right)^{-1-k} .
$$

(b) If $\varphi, \psi$, and $\eta$ are normalized bump functions, then $K$ satisfies the following cancellation conditions:

$$
\begin{aligned}
\left|\int_{\mathbb{R}} \partial_{z}^{j} K(z, t) \varphi(\delta t) d t\right| & \leq C_{j}|z|^{-2-j}, \\
\left|\int_{\mathbb{C}} \partial_{t}^{k} K(z, t) \psi(\delta z) d z\right| & \leq C_{k}|t|^{-1-k}, \\
\left|\int_{\mathbb{R}} K(z, t) \eta\left(\delta_{1} z, \delta_{2} t\right) d z d t\right| & \leq C
\end{aligned}
$$

where the constants are independent of the normalized bump functions and the positive parameters $\delta, \delta_{1}, \delta_{2}$.

Conversely, if $K$ is a distribution on $\mathbb{H}^{1}$ which is radial in $z$ and satisfies (a) and (b), then $K * f=m(\mathcal{L}, T) f$ where $m$ satisfies (II.4.2).

II.4.3. Singular integrals with flag kernels. The kernels appearing in Stein's work with Müller and Ricci are not pseudolocal since they have singularities on a (single) proper nontrivial subspace. On the other hand, the singular set has a simpler structure than that of a general product kernel (for the double Hilbert transform in $\mathbb{R}^{2}$ it is the union of the two coordinate axes). Kernels of this kind appear in many situations, e.g., when composing a standard Calderón-Zygmund kernel $K_{1}(x, y)$ on $\mathbb{R}^{n+m}$ with $K_{2}(x) \delta_{0}(y), K_{2}$ being a Calderón-Zygmund kernel on $\mathbb{R}^{n}$.

The notion of flag kernel was introduced and studied in two papers [188] and [212] by Nagel, Ricci, Stein, and Wainger in order to provide a general framework for studying kernels of this kind and their composition properties. Flag kernels are examples of product kernels, but they are more flexible and are more appropriate when considering convolution operators on nilpotent groups.

To define flag kernels, for $1 \leq j \leq n$, let $W_{j}$ be a subspace of $\mathbb{R}^{N}$ equipped with a one-parameter family of dilations $D_{t}^{j}: W_{j} \rightarrow W_{j}$ of homogeneous dimension $Q_{j}$. Suppose that $\mathbb{R}^{N}=W_{1} \oplus \cdots \oplus W_{n}$. If $V_{j}=W_{1} \oplus \cdots \oplus W_{j}$, then $(0)=$ $V_{0} \subset V_{1} \subset \cdots \subset V_{n-1} \subset V_{n}=\mathbb{R}^{N}$ is a flag. If $\mathbf{x}_{j} \in W_{j}$, let $\left|\mathbf{x}_{j}\right|$ be a norm which is homogeneous with respect to $D_{t}^{j}$. Then a flag kernel adapted to the flag $\left\{V_{j}\right\}$ is a distribution $K$ on $\mathbb{R}^{N}$ which is $\mathcal{C}^{\infty}$ away from the subspace $\mathbf{x}_{n}=\mathbf{0}$ and which satisfies certain differential inequalities and cancellation conditions. The inequalities are easy to state:

$$
\left|\partial_{\mathbf{x}_{1}}^{\alpha_{1}} \cdots \partial_{\mathbf{x}_{n}}^{\alpha_{n}} K(\mathbf{x})\right| \leq C_{\alpha} \prod_{j=1}^{n}\left(\left|\mathbf{x}_{j}\right|+\cdots+\left|\mathbf{x}_{n}\right|\right)^{-Q_{j}-\left|\alpha_{j}\right|} .
$$

The cancellation conditions are more delicate, but are analogues of the inequalities in equation (II.4.3). The results on flag kernels and convolution operators in the papers [188] and [212] are of several types.

1. In addition to the definition in terms of differential inequalities and cancellation conditions, flag kernels can be characterized in terms of estimates on their Euclidean Fourier transforms and in terms of decompositions into dyadic sums. 
2. If $\mathbb{R}^{N}$ has the structure of a nilpotent Lie group $G$, under appropriate compatibility assumptions between the subspaces $W_{j}$ with their dilations and the group law, the convolution operator $f * K$ with a flag kernel $K$ is bounded on $L^{p}(G)$ for $1<p<\infty$.

3. Operators given by convolution with flag kernels form an algebra under composition.

4. The class of flag kernels is closed under passage to a quotient subgroup. This is not true for product kernels, but every product kernel can be written as a finite sum of flag kernels

Flag kernels also arise in various problems in several complex variables.

5. If $\Gamma \subset \mathbb{R}^{n}$ is a proper polyhedral cone with nonempty interior, and if $\Omega=\mathbb{R}^{n}+i \Gamma$ is the tube over $\Gamma$ with Shilov boundary $\Omega_{0}=\mathbb{R}^{n}+i \mathbf{0}$, the orthogonal projection of $L^{2}\left(\Omega_{0}\right)$ onto the closed subspace of boundary values of holomorphic functions (the Szegö projection) is a sum of a finite number of flag kernels with singularities along the faces of the cone.

6. Certain quadratic CR submanifolds $\Sigma \subset \mathbb{C}^{n}$ carry a Lie group structure $G_{\Sigma}$ compatible with their complex imbedding, and there is a Marcinkiewicz multiplier theorem for these groups which extends the results of Müller, Ricci, and Stein [168]. The resulting operators are finite sums of convolution operators with flag kernels.

7. The Szegö projection and certain derivatives of the fundamental solution to the Kohn Laplacian $\square_{b}$ on the submanifolds are given by sums of convolution operators with flag kernels.

II.4.4. Estimates for $\square_{b}$ on decoupled domains. Motivated perhaps by his observation of the important role of the Heisenberg group in problems in several complex variables, Stein also worked on one-parameter problems involving general nilpotent groups and also on one-parameter problems in complex analysis. Thus in a series of papers [139], [140], [145], Ricci and Stein consider singular integrals on nilpotent groups whose kernels have oscillatory factors and are supported on lower-dimensional submanifolds on nilpotent groups.

Stein and his collaborators also successfully applied variants of the classical oneparameter Calderón-Zygmund theory of singular integrals to study function theory on certain classes of domains in several complex variables. This includes his work with Folland [73] on estimates for the $\bar{\partial}_{b}$-complex on strictly pseudoconvex domains in $\mathbb{C}^{n}$; his work with Nagel, Rosay, and Wainger [141], [143] on estimates for the Szegö kernel in domains of finite type in $\mathbb{C}^{2}$; and for the same class of domains, construction, and estimates for a parametrix of the $\bar{\partial}$-Neumann problem in work with Chang and Nagel [142], [156]. Some of these results were later generalized by Stein's student Ken Koenig to the class of domains whose Levi form have comparable eigenvalues $[\mathrm{Ko}$. For these domains there is a natural nonisotropic one-parameter distance or pseudometric on the boundary such that estimates of the integral kernels of the relevant operators are given in terms of this metric and the volumes of the associated family of balls.

A domain $\Omega \subset \mathbb{C}^{n+1}$ is decoupled if there are subharmonic, nonharmonic polynomials $P_{j}: \mathbb{C} \rightarrow \mathbb{R}$ so that $\Omega=\left\{\left(z_{1}, \ldots, z_{n+1}\right) \in \mathbb{C}^{n+1}: \operatorname{Im} z_{n+1}>\sum_{j=1}^{n} P_{j}\left(\mathbf{z}_{j}\right)\right\}$. Identifying $\left(\mathbf{z}, t+i \sum_{j=1}^{n} P_{j}\left(z_{j}\right)\right) \in \partial \Omega$ with $(\mathbf{z}, t) \in \mathbb{C}^{n} \times \mathbb{R}$, the operators $\partial_{b} f(\mathbf{z})=$ $\sum_{j=1}^{n} Z_{j} f(\mathbf{z}) d \mathbf{z}_{j}$ and $\bar{\partial}_{b} f(\mathbf{z})=\sum_{j=1}^{n} \bar{Z}_{j} f(\mathbf{z}) d \overline{\mathbf{z}}_{j}$ are the tangential components of 
the operators $\partial$ and $\bar{\partial}$. Here $Z_{j}=\frac{\partial}{\partial z_{j}}+i \frac{\partial P_{j}}{\partial z_{j}}\left(z_{j}\right) \frac{\partial}{\partial t}$ and $\bar{Z}_{j}=\frac{\partial}{\partial \bar{z}_{j}}-i \frac{\partial P_{j}}{\partial \bar{z}_{j}}\left(z_{j}\right) \frac{\partial}{\partial t}$. The tangential Kohn Laplacian is then the second-order system of operators $\square_{b}=$ $-\partial_{b} \bar{\partial}_{b}-\bar{\partial}_{b} \partial_{b}$ acting on $(0, q)$-forms on the boundary. $\square_{b}$ acts diagonally on the coefficients of the form, and one is led to study the $2^{n}$ second-order operators $\square_{b}^{j, k}=W_{j} \bar{W}_{j}+\bar{W}_{k} W_{k}$, where each $W_{l}$ is either $Z_{l}$ or $\bar{Z}_{l}$ and $\bar{W}_{l}$ is the other choice. It follows from [66] that if $P_{j}\left(z_{j}\right)=\left|z_{j}\right|^{2}$ (and more generally for strictly pseudoconvex domains), there is a fundamental solution $G_{j, k}(\mathbf{p}, \mathbf{q})$ for $\square_{b}^{j, k}$ which satisfies

$$
\left|Z^{\boldsymbol{\alpha}} \bar{Z}^{\boldsymbol{\beta}} G_{j, k}(\mathbf{p}, \mathbf{q})\right| \lesssim d(\mathbf{p}, \mathbf{q})^{2-|\boldsymbol{\alpha}|-|\boldsymbol{\beta}|} V((\mathbf{z}, t) ; d(\mathbf{p}, \mathbf{q}))^{-1},
$$

where $d(\mathbf{p}, \mathbf{q})$ is the nonisotropic distance between $\mathbf{p}$ and $\mathbf{q}$, and $V(\mathbf{p} ; \delta)$ is the volume of the nonisotropic ball centered at $\mathbf{p}$ of radius $\delta$. Analogous results for domains of finite type in $\mathbb{C}^{2}$ were obtained by Fefferman and Kohn [FK1], [FK2] and by Christ [Ch2, Ch3, Ch6]. The case of domains whose Levi form has comparable eigenvalues was treated by Koenig [Ko. Thus for these domains the fundamental solution is controlled by a single one-parameter family of balls.

However the situation for decoupled domains in $\mathbb{C}^{n}$ for $n \geq 3$ is very different. Let $D_{k, 2}=\left\{\left(z_{1}, z_{2}, z_{3}\right) \in \mathbb{C}^{3}: \operatorname{Im} z_{3}>\left|z_{1}\right|^{2 k}+\left|z_{2}\right|^{2}\right\}$. When $k>1$, Machedon [Ma] constructed a parametrix $K$ for the operator $\bar{Z}_{1} Z_{1}+Z_{2} \bar{Z}_{2}$ for which

$$
|K(\mathbf{p}, \mathbf{q})| \lesssim \frac{d_{1}^{2}(\mathbf{p}, \mathbf{q})}{\left|B_{1}\left(\mathbf{q}, d_{1}(\mathbf{p}, \mathbf{q})\right)\right|} \log \left(2+\frac{d_{1}(\mathbf{p}, \mathbf{q})}{d_{2}(\mathbf{p}, \mathbf{q})}\right)
$$

where $d_{1}, d_{2}$ are two different noncomparable distances on the boundary, and he showed that this estimate cannot be improved. One is thus forced to deal with a two-parameter family of balls, and the difference with the strictly pseudoconvex case is that now the eigenvalues of the Levi form are $k^{2}\left|z_{1}\right|^{2 k-2}$ and 1 , which are not comparable. Machedon's argument relied on the fact that the Levi form has only one vanishing eigenvalue.

In a series of papers [189], [190], [196], [200], Nagel and Stein generalized and extended Machedon's work to the case of general decoupled domains. The ultimate objective was to construct fundamental solutions for the operators $\mathcal{L}_{j, k}$, estimate these fundamental solutions, and obtain sharp $L^{p}$ and Lipschitz estimates for appropriate derivatives of the fundamental solution. Consider the example $D_{n, m}=\left\{\left(z_{1}, z_{2}, z_{3}\right) \in \mathbb{C}^{3}: \operatorname{Im} z_{3}>\left|z_{1}\right|^{n}+\left|z_{2}\right|^{m}\right\}$ with $n \leq m$ even integers. Let $M$ denote the boundary of $D_{n, m}$, and write $\mathbf{p}=(\mathbf{z}, t), \mathbf{q}=(\mathbf{w}, s) \in \partial D_{n, m}$. Then the eigenvalues of the Levi form are $\lambda_{1}(\mathbf{p}) \approx\left|z_{1}\right|^{n-2}$ and $\lambda_{2}(\mathbf{p}) \approx\left|z_{2}\right|^{m-2}$. Focusing on the operator $\square_{b}=Z_{1} \bar{Z}_{1}+\bar{Z}_{2} Z_{2}$, the main result in this case is the following.

Theorem II.4.2. There is an operator $\mathcal{K} f(\mathbf{p})=\int_{\partial D_{n, m}} K(\mathbf{p}, \mathbf{q}) f(\mathbf{q}) d \mathbf{q}$ so that $\mathcal{K} \square_{b}=\square_{b} \mathcal{K}=I$ when applied to smooth functions with compact support.

(a) There are estimates for the size of $K(\mathbf{p}, \mathbf{q})$ and its derivatives analogous to (II.4.4) which involve two noncomparable distances $d_{\Sigma}$ and $d_{S}$. If $\mathbf{p}=$ $\left(z_{1}, z_{2}, t\right)$ and $\mathbf{0}=(0,0,0)$, then

$$
\begin{aligned}
& d_{\Sigma}(\mathbf{0}, \mathbf{p})=\left|z_{1}\right|+\left|z_{2}\right|+|t|^{\frac{1}{m}}, \\
& d_{S}(\mathbf{0}, \mathbf{p})=\left|z_{1}\right|^{m}+\left|z_{2}\right|^{n}+|t| .
\end{aligned}
$$

(b) The operators $Z_{1} \bar{Z}_{1} \mathcal{K}, \bar{Z}_{2} Z_{2} \mathcal{K}, \bar{Z}_{1} \bar{Z}_{1} \mathcal{K}, Z_{2} Z_{2} \mathcal{K}$ are bounded on $L^{p}(M)$ for $1<p<\infty$ 
(c) Let $B_{1}, B_{2}$ be bounded functions on $M$ such that $\lambda_{1}(\mathbf{p}) B_{1}(\mathbf{P}) \lesssim \lambda_{2}$ (p) and $\lambda_{2}(\mathbf{p}) B_{2}(\mathbf{P}) \lesssim \lambda_{1}(\mathbf{p})$. Then the operators $B_{1} \bar{Z}_{1} Z_{1} \mathcal{K}$ and $B_{2} Z_{2} \bar{Z}_{2} \mathcal{K}$ are bounded on $L^{p}(M), 1<p<\infty$.

(d) Let $B_{1}, B_{2}$ be bounded functions on $M$ such that $B_{1}(\mathbf{P}) \lesssim \lambda_{2}(\mathbf{p})$ and $B_{2}(\mathbf{P}) \lesssim \lambda_{1}(\mathbf{p})$. Then the operators $B_{1} Z_{1} Z_{1} \mathcal{K}$ and $B_{2} \bar{Z}_{2} \bar{Z}_{2} \mathcal{K}$ are bounded on $L^{p}(M)$ for $1<p<\infty$.

(e) The operator $\mathcal{K}$ maps $L^{\infty}(M)$ to the isotropic Hölder space $\Lambda^{\alpha}(M)$ where $\alpha=\min \left\{\frac{2}{n}, \frac{2}{m}\right\}$.

The proof of $L^{p}$-regularity does not involve using the size estimates of the kernel, but rather proceeds as follows. Let $D_{n}=\left\{\left(z_{1}, w_{1}\right): \operatorname{Im} w_{1}>\left|z_{1}\right|^{n}\right\}$ and $D_{m}=$ $\left\{\left(z_{2}, w_{2}\right): \operatorname{Im} w_{2}>\left|z_{2}\right|^{m}\right\}$ with boundaries $M_{1}$ and $M_{2}$. Let $\widetilde{M}=M_{1} \times M_{2}$ and let $\pi\left(\left(z_{1}, t_{1}\right),\left(z_{2}, t_{2}\right)\right)=\left(z_{1}, z_{2}, t_{1}+t_{2}\right)$ so that $\pi: \widetilde{M} \rightarrow M$. Regularity results for $\square_{b}$ on $M$ are obtained from regularity results on each factor $M_{j}$. Passing to the product allows one to consider combinations of the separate metrics on each factor, and this leads to the different metrics on $M$.

We note that the process of lifting the analysis from $M$ to $\widetilde{M}$ is analogous to the procedure for obtaining $L^{p}$-boundedness of Marcinkiewicz multipliers described earlier.

II.4.5. Algebras of operators controlled by multiple norms. The memoir [224] by Nagel, Ricci, Stein, and Wainger returns to questions raised in the paper of Phong and Stein [107] and studies algebras of singular integral operators on nilpotent Lie groups that arise when considering compositions of Calderón-Zygmund operators with different homogeneities. The relevant kernels do reflect a multiparameter structure, but unlike product kernels or flag kernels, they are singular only at the origin, and so the associated convolution operators are pseudolocal.

The following is an example. Write $\mathbb{R}^{N}=\mathbb{R}^{c_{1}} \oplus \cdots \oplus \mathbb{R}^{c_{n}}$, and write $\mathbf{x}=$ $\left(x_{1}, \ldots, x_{N}\right)$ as $\left(\mathbf{x}_{1}, \ldots, \mathbf{x}_{n}\right)$ with $\mathbf{x}_{j} \in \mathbb{R}^{c_{j}}$. Consider the isotropic one-parameter family of dilations on $\mathbb{R}^{N}$ given by $\lambda \cdot \mathbf{x}=\left(\lambda x_{1}, \ldots, \lambda x_{N}\right)$. (More general nonisotropic dilations are also possible.) Let $\left|\mathbf{x}_{j}\right|$ be the corresponding homogeneous norm on $\mathbb{R}^{c_{j}}$, and let

$$
N_{j}\left(\mathbf{x}_{1}, \ldots, \mathbf{x}_{n}\right)=\left|\mathbf{x}_{1}\right|^{e(j, 1)}+\cdots+\left|\mathbf{x}_{n}\right|^{e(j, n)},
$$

where $\{e(j, k)\}$ are positive and satisfy $e(j, j)=1$ and $e(j, l) \leq e(j, k) e(k, l)$ for $1 \leq j, k, l \leq n$. Note that $N_{j}$ is a homogeneous norm for the family of dilations

$$
\delta_{j}(\lambda)\left(\mathbf{x}_{1}, \ldots, \mathbf{x}_{n}\right)=\left(\lambda^{\frac{1}{e(j, 1)}} \mathbf{x}_{1}, \ldots, \lambda^{\frac{1}{e(j, n)}} \mathbf{x}_{n}\right) .
$$

Let $\mathbf{E}=\{e(j, k)\}$. The class $\mathcal{P}(\mathbf{E})_{0}$ then consists of distributions $K$ on $\mathbb{R}^{N}$ which are smooth away from the origin, decay rapidly along with their derivatives at infinity, and satisfy differential inequalities and cancellation conditions. The cancellation conditions are quite technical, but the size conditions are easy to state:

$$
\left|\partial^{\gamma} K\left(\mathbf{x}_{1}, \ldots, \mathbf{x}_{n}\right)\right| \leq C_{\gamma} \prod_{j=1}^{n} N_{j}\left(\mathbf{x}_{1}, \ldots, \mathbf{x}_{n}\right)^{-c_{j}-\gamma_{j}} .
$$

Thus, although not homogeneous, $K$ and its derivatives are controlled by $n$ different homogeneities. There are the following kinds of results for such kernels. 
1. Kernels in $\mathcal{P}(\mathbf{E})_{0}$ can also be characterized in terms of their Euclidean Fourier transform, and in terms of decompositions into dyadic sums of normalized bump functions.

2. If the rank of the matrix $\mathbf{E}$ is equal to 1, then the class of distributions $\mathcal{P}(\mathbf{E})_{0}$ essentially coincides with a class of nonisotropic Calderón-Zygmund kernels. If the rank is greater than 1, these kernels fall outside the class of standard Calderón-Zygmund kernels.

3. Kernels which are flag kernels for two opposite flags belong to an appropriate class $\mathcal{P}(\mathbf{E})_{0}$.

4. If $G$ is a homogeneous nilpotent Lie group, and if the automorphic dilations on $G$ are appropriately compatible with the matrix $\mathbf{E}$, then convolution on $G$ with a kernel $K \in \mathcal{P}(\mathbf{E})_{0}$ defines a bounded operator on $L^{p}(G)$ for $1<p<\infty$.

5. The collection of such operators forms an algebra under composition.

6. The smallest algebra of convolution operators generated by Calderón-Zygmund operators with different homogeneities can be characterized using the algebras $\mathcal{P}(\mathbf{E})_{0}$.

II.4.6. A personal note. Over a span of many years each of us had the privilege and pleasure of collaborating with Eli Stein on a variety of mathematical problems. We are deeply grateful for his continuing friendship and support, and for the opportunity to experience at close hand his fantastic ability to find fertile ground for mathematical research, his wide ranging interests which often found deep connections between different areas, and his talent for grounding general theories in concrete examples.

\section{II.5. Singular RAdon transforms AND the $\bar{\partial}$-NeUmann PROBlem}

contributed by D. Phong

For many years already, the $\bar{\partial}$ problem had been central to Eli's vision of a theory of singular integrals as the common thread between many seemingly distant areas of Fourier analysis, complex analysis, partial differential equations, and nilpotent Lie groups. By the mid-1970s, partly inspired by the $\bar{\partial}_{b}$ problem, he had developed a far-reaching theory which encompassed, in its various forms, singular integrals on strongly pseudoconvex boundaries (with G. Folland), sharp estimates for the sums of squares of vector fields (with L. Rothschild), and an $L^{p}$ theory for inhomogeneous classes of pseudodifferential operators (with A. Nagel). What was still lacking was a singular integral theory for the $\bar{\partial}$-Neumann problem. In his 1976 joint work with P. Greiner, Eli had circumvented this difficulty and obtained $L^{p}$-estimates, by decomposing the solution into a product of several operators of different known types, each of which can be estimated separately. Only when an explicit formula for the Green's function for the $\bar{\partial}$ operator became available in 1979 [Ph], $\mathrm{BFG}$ ] was it possible to consider directly the development of an $L^{p}$-theory for the corresponding singular integrals. Eli turned to this task in joint work with me.

The Green's function for the $\bar{\partial}$-Neumann problem did not fall into any existing theory of singular integrals at that time because it was a product of kernels with two different kinds of homogeneity. When restricted to the boundary, it was 
comparatively easy to estimate [107], but on the domain itself, it presented the additional complication of being smoothing in different ways on and off the boundary. Altogether, it seemed to present an inextricable entanglement.

The key discovery in [122], [132], and [133] is that the entanglement can be unraveled by introducing a new notion of operator on the level sets of the domain, called a singular Radon transform, and writing the Green's function as a Hilbert integral operator valued in the space of singular Radon transforms. More precisely, let $M$ be a compact smooth manifold, and assume that at each point $x \in M$, there is a smooth hypersurface $H_{x}$, equipped with a Calderon-Zygmund kernel $K(x, \cdot)$ and a density $d \mu_{x}(\cdot)$, all varying smoothly with $x$. Define the corresponding singular Radon transform $R(K)$ by

$$
C^{\infty}(M) \ni f \rightarrow(R(K) f)(x)=\int_{H_{x}} K(x, y) f(y) d \mu_{x}(y) \in C^{\infty}(M) .
$$

We shall be particularly interested in distributions $\left\{H_{x}\right\}_{x \in M}$, which have nonvanishing rotational curvature. This means that, near the diagonal $x=y$, the normal bundle $N^{*}(\mathcal{C}) \subset T^{*}(M) \times T^{*}(M)$ of the manifold $\mathcal{C}=\{(x, y) \in M \times M ; y \in$ $\left.H_{x}\right\}$ projects on each of the two factors $T^{*}(M)$ with injective differentials. This is the condition for optimal regularity of the class of Fourier integral operators with $N^{*}(\mathcal{C})$ as their Lagrangian. The main results of [122], [132], [133], [144] are as follows:

Theorem II.5.1. Let $M$ be a compact smooth manifold, with a distribution of hypersurfaces $H_{x}$ satisfying the nonvanishing rotational curvature condition. For any smooth distribution $\{K(x, \cdot)\}$ of Calderón-Zygmund kernels, the corresponding singular Radon transform (II.5.1) is bounded in $L^{p}(M)$ for any $1<p<\infty$.

Consider now a strongly pseudoconvex domain $D$, equipped with a Levi metric. Operators on the Siegel upper half-space $U=H_{n} \times \mathbf{R}_{+}$, where $H_{n}$ is the Heisenberg group, can be transplanted to operators on $D$ as follows. Let $\rho(z)$ be the geodesic distance to $\partial D$. For each $\varepsilon>0$ small enough and $z \in D$ with $\rho(z)=\varepsilon$, there are holomorphic coordinates $\left(\zeta, \zeta_{n+1}\right)$ centered at $z$ so that for $w$ near $z, \rho(w)$ is given by $\rho(w)=\varepsilon+\operatorname{Im} \zeta_{n+1}-|\zeta|^{2}+O\left(\left|\zeta_{n+1}\right||\zeta|+\rho|\zeta|^{2}+|\zeta|^{3}\right)$. We identify $w$ with $w=\left(\zeta, t=\operatorname{Re} \zeta_{n+1}, \rho(w)\right)$ and view it as a point in $U$. It is convenient to introduce the notation

$$
\Theta(z, w)=(\zeta, t)
$$

and to think of $\Theta(z, w)$ as an element of the Heisemberg group. Any kernel $\mathcal{K}(\zeta, t, \rho)$ on the upper half-space $U$ now defines an operator on $D$ by

$$
(\mathrm{Op}(\mathcal{K}) f)(z)=\int_{U} \mathcal{K}(\Theta(z, w), \rho(z)+\rho(w)) \chi(z, w) f(w) d V(w)
$$

where $d V(w)$ is the measure on $D$, and $\chi(z, w)$ is a fixed cut-off function, which is 1 in a small neighborhood of the diagonal $z=w$ near $\partial D$ and 0 outside another small neighborhood.

The parametrix for the $\bar{\partial}$-Neumann problem is a linear combination of operators of the form (II.5.3), with each kernel $\mathcal{K}(\zeta, t, \rho)$ given by $E_{k}(\zeta, t, \rho), H_{\ell}(z, t, \rho)$, with $E_{k}, H_{\ell}$ smooth outside of 0 and homogeneous of degrees $k$ and $\ell$, respectively, with respect to the two dilations $\lambda \cdot(\zeta, t, \rho)=(\lambda \zeta, \lambda t, \lambda \rho)$ and $\lambda \cdot(\zeta, t, \rho)=\left(\lambda \zeta, \lambda^{2} t, \lambda^{2} \rho\right)$ Ph, BFG. Sharp smoothing estimates for the parametrix are obtained by differentiating, resulting in operators whose kernels are still of the same form, but with 
lower degrees of homogeneity. It turns out that all the resulting operators can be easily seen to satisfy the desired bounds, except in the critical case of $k=2 n$, $\ell=4$, with $\mathcal{K}(z, t, \rho)$ satisfying the Calderón-Zygmund cancellation property as a function of $z$. We have then:

Theorem II.5.2. Under the conditions just stated on the kernel $\mathcal{K}$, the operator (II.5.3) is bounded on $L^{p}(D)$ for any $1<p<\infty$.

This follows by viewing (II.5.3) as a Hilbert integral operator valued in a space of suitable singular Radon transforms. More concretely, we consider the hypersurfaces $S_{\rho}=\{z \in D ; \rho(z)=\rho\}$ together with the distribution $\left\{H_{z}\right\}_{z \in S_{\rho}}$ defined by $H_{z}=$ $\left\{w \in S_{\rho} ; t=0\right\}$ in the coordinate system $(\zeta, t, \rho)$ centered at $z$. For $\rho$ small, the condition that this distribution of hypersurfaces have nonvanishing rotational curvature follows from the strong pseudoconvexity of the domain $D$. Let $N$ and $T$ be the normal and tangent vector fields, respectively, to the level sets $S_{\rho}$. Let $\iota_{\mu}{ }^{\rho}: S_{\rho} \rightarrow S_{\mu}$ be the diffeomorphism obtained by following the integral curves of $N$. We can then write

$$
(\operatorname{Op}(\mathcal{K}) f)(z)=\int_{0}^{\infty} \int_{-\infty}^{\infty} \mathrm{R}_{\tau, \rho, \mu} \cdot \tilde{f}_{\tau, \rho, \mu} d \tau d \mu, \quad z \in D, \rho=\rho(z),
$$

where $\tilde{f}_{\tau, \rho, \mu}$ is the function on $S_{\rho}$ defined by $\tilde{f}_{\tau, \rho, \mu}(w)=f\left(\iota_{\mu}{ }^{\rho}\left(e^{\tau T} w\right)\right), w \in S_{\rho}$, and $\mathrm{R}_{\tau, \rho, \mu}$ is the singular Radon transform on $S_{\rho}$ associated to the distribution $\left\{H_{z}\right\}_{z \in S_{\rho}}$ with Calderón-Zygmund kernel $K(z, w)=\mathcal{K}\left(\Theta\left(z, e^{\tau T} w\right), \rho+\mu\right)$. By Theorem II.5.1, the operators $\mathrm{R}_{\tau, \rho, \mu}$ are bounded in norm by $C\left(\rho^{2}+\mu^{2}+\tau^{2}\right)^{-1}$, and Theorem $\amalg .5 .2$ follows by standard estimates for Hilbert integrals.

At the time it appeared, Theorem $\amalg .5 .1$ brought in the latest techniques in analysis, including group Fourier transforms for the model case of the Heisenberg group [123], twisted convolutions, and the theory of $S_{1 / 2,1 / 2}$ pseudodifferential operators with limited regularity of Coifman and Meyer [CM]. The concept of singular Radon transforms itself, which arises here from the $\bar{\partial}$-Neumann problem, is a generalization of the Hilbert transform along curves, introduced by A. Nagel, N. Riviere, and S. Wainger [NRW1]. It has appeared frequently in both analysis and geometry since. Extensions to the discrete setup, to fractional kernels instead of CalderonZygmund, as well as $L^{p}-L^{q}$ smoothing properties have also been considered by many authors. The literature is too extensive to describe more fully here, but see, e.g., Ionescu and Wainger [IW] and Tao and Wright [TW] and references therein.

As pointed out afterwards by A. Greenleaf and G. Uhlmann [GrU], the singular Radon transform of Theorem II.5.1 can also be viewed as a Fourier integral operator associated to a pair of intersecting Lagrangians, which are in this case the diagonal in $T^{*}(M) \times T^{*}(M)$ and the normal bundle of the canonical relation $\mathcal{C}$. Such operators arose in work of Guillemin and Uhlmann GU] and have also led to many interesting developments.

Theorem II.5.1 has been considerably generalized and simplified since its original appearance. In particular, a version with almost no condition on rotational curvature, besides its nonvanishing of infinite order, was established in 1999 by Eli in joint work with M. Christ, A. Nagel, and S. Wainger [182]. The Green's function for the $\bar{\partial}$-Neumann problem and the inverse of the operator $\square_{+}^{-1}$ obtained in [107] can be considered as cases when the kernels of the composition of operators of different homogeneities can be worked out explicitly. This general problem has 
been of lasting interest to Eli, and he returned to it more recently with P. L. Yung [217].

II.5.1. Oscillatory integral operators with general phases. The generalized Radon transforms defined by hypersurfaces considered in Theorem II.5.1 attracted Eli's attention to averages over more general submanifolds. The rotational curvature condition will not generically hold for higher codimensions, and it becomes necessary to refine the standard van der Corput lemma and method of stationary phase to reflect degenerate phases and the dependence on external parameters.

By the mid-1990s, Eli had gained a considerable understanding of oscillatory integral operators with degenerate phases, with model cases with polynomial phases considered in [144], [166]. But there were still some major difficulties, including what was a proper geometric formulation, as well as how to decompose operators in the presence of degeneracies. So the present theorem was a breakthrough in his program [174]:

Theorem II.5.3. Consider the oscillatory integral operator defined by

$$
C_{0}^{\infty}(\mathbf{R}) \ni f \rightarrow(T f)(x)=\int_{-\infty}^{\infty} e^{i \lambda S(x, y)} \chi(x, y) f(y) d y \in C_{0}^{\infty}(\mathbf{R}),
$$

where $S(x, y)$ is an analytic phase function and $\chi \in C_{0}^{2}\left(\mathbf{R}^{2}\right)$ is supported near the origin. Let $\delta$ be the Newton decay rate of $S(x, y)$ at the origin, as defined below. Then if the support of $\chi$ is sufficiently small, we have the decay rate,

$$
\|T\|_{L^{2} \rightarrow L^{2}} \leq C|\lambda|^{-\delta / 2} \text {. }
$$

Furthermore, if $\chi(0) \neq 0$, we also have $\|T\|_{L^{2} \rightarrow L^{2}} \geq c|\lambda|^{-\delta / 2}$ for some $c>0$.

To define the Newton decay rate $\delta$, we consider the set of integer vertices $(p, q)$ with $p>0, q>0$, where the monomial $x^{p} y^{q}$ occurs in the Taylor expansion at 0 of the phase $S(x, y)$ with nonzero coefficient. Then $\delta:=\min \delta_{\ell}$, where $\ell$ denotes a boundary line of the convex hull of the upper quadrants with corner at these vertices, and $\left(\delta_{\ell}^{-1}, \delta_{\ell}^{-1}\right)$ is the intersection of $\ell$ with the diagonal $p=q$. Note that the same construction, but allowing all vertices, gives the usual notion of Newton distance, which was shown by Varchenko Va to give the decay rate of scalar and generic oscillatory integrals with phase $S(x, y)$. This notion cannot be the right one for oscillatory integral operators, whose norm is clearly independent of the vertices with either $p=0$ or $q=0$. It is also valid only generically, while the estimate in Theorem $\amalg .5 .3$ is valid for all phases $S(x, y)$.

The proof of Theorem II.5.3 requires a decomposition of $T$ into operators supported on curved boxes $\mathcal{B}$ where $\left|S_{x y}^{\prime \prime}\right| \sim \mu$ for various scales $\mu$. Because the shapes and sizes of $\mathcal{B}$ can vary, e.g.,

$$
\mathcal{B}=\{(x, y) ; \phi(x)<y<\phi(x)+\gamma, \alpha<x<\beta\},
$$

and the possible (in)dependence on the parameters $\phi, \alpha, \beta, \gamma$ has to be carefully accounted for, the decomposition into the boxes $\mathcal{B}$ themselves is obtained by examining the roots of $S_{x y}^{\prime \prime}$ and the successive clusters that they can form at different scales.

Theorem II.5.3 has been extended in many directions since (e.g., to smooth phases [Gs, Ry] to more general classes of operators [Se or to higher dimensions GPT, CGP, Gs, GX. Its resolution methods have been applied to give an independent proof [179] of Karpushkin's stability theorem [Ka] without the use of versal 
deformation theory, and to restriction phenomena and maximal estimates for hypersurfaces in $\mathbf{R}^{3}$; see [BDIM, IKM].

With degenerate phases, stability issues acquire particular importance. In joint work with Phong and J. Sturm, Eli [187] subsequently obtained the following theorem, which is a strengthening of Theorem II.5.3 to stable estimates and the multilinear setting:

Theorem II.5.4. Let $S\left(x_{1}, \ldots, x_{d}\right)$ be a polynomial in $d$ variables of degree $n_{S}$, $d \geq 2$. Let $\alpha^{(1)}, \ldots, \alpha^{(K))}$ be $K$ nonnegative integer vertices in $\mathbf{R}^{d}$, each of which has at least two strictly positive components, and let

$$
D\left(\alpha^{(1)}, \ldots, \alpha^{(K)}\right)=\left\{x \in \mathbf{R}^{d} ; \max _{m}\left|x_{m}\right|<1 \text { and }\left|D_{x}^{\alpha^{(j)}} S(x)\right| \geq 1,1 \leq j \leq K\right\} .
$$

Consider the integral operator

$$
T\left(f_{1}, \ldots, f_{d}\right)=\int_{D} e^{\lambda S\left(x_{1}, \cdots, x_{d}\right)} \prod_{m=1}^{d} f_{m}\left(x_{m}\right) \prod_{m=1}^{d} d x_{m}
$$

where $D$ is any $(N, n, r)$-algebraic subset of $D\left(\alpha^{(1)}, \ldots, \alpha^{(K)}\right)$, in the sense that it is a union of at most $N$ sets, each of which is the intersection of at most $r$ sublevel sets of nonconstant polynomials of degrees bounded by $n$. Then for any multi-index $\alpha=\left(\alpha_{1}, \ldots, \alpha_{d}\right)$ in the convex hull with corners at the $\alpha^{(j)}$, we have

$$
\left|T\left(f_{1}, \ldots, f_{d}\right)\right| \leq C|\lambda|^{-1 /|\alpha|} \log ^{d-1 / 2}(2+|\lambda|) \prod_{m=1}^{d}\left\|f_{m}\right\|_{L^{p_{m}}},
$$

where $1-\frac{1}{p_{m}}=\frac{\alpha_{m}}{|\alpha|}$, and the constant $C$ depends only on $d, n_{S}$, and $\left(N_{D}, n, r\right)$ of $D$. When $d=2$ and the integral over $D$ in (II.5.9) is replaced by an integral with $a C^{2}$ function $\chi$ supported in the unit cube, then all the log factors in (II.5.10) can be eliminated, and we recover Theorem II.5.3, with constants now uniform in the phase $S$.

Theorem II.5.4 is proved by introducing a completely different decomposition than before, into curved trapezoids instead of curved boxes. This also yields a version of Theorem II.5.4 for stable estimates of the volume of sublevel sets, generalizing an earlier estimate of Carbery, Christ, and Wright [CCW].

\section{II.6. Polynomial Carleson operators} contributed by L. Pierce

Fourier's foundational work firmly established the utility of the Fourier series associated to a function, but left open many interesting questions of convergence: what conditions on the original function, or alternative methods of summation, guarantee pointwise convergence of the Fourier series? These questions were taken up in various forms by Dirichlet, Fejér, Riemann, Weierstrass, Dedekind, du BoisReymond, and others (see, e.g., [Kö]). In 1913, in the context of Lebesgue measure, Luzin articulated a landmark question $[\mathrm{Lu}]$ : does the series converge pointwise almost everywhere if $f$ is an $L^{2}$ function? After a surprising counterexample in $L^{1}$ by Kolmogorov [Kl], in contrast the $L^{2}$ question was famously resolved in the affirmative by Carleson $\mathrm{Cr}$. Define for each $\lambda \in \mathbb{R}$ the following operator, analogous 
to taking a partial sum of the Fourier series:

$$
T_{\lambda} f(x)=\text { p.v. } \int_{[-\pi, \pi]} f(x-y) e^{i \lambda y} \frac{d y}{y} .
$$

Then Carleson's theorem is that $f \mapsto \sup _{\lambda \in \mathbb{R}}\left|T_{\lambda} f(x)\right|$ satisfies a weak- $L^{2}$ bound, from which it follows that even worst possible behavior of partial sums can be controlled, at least pointwise almost everywhere. Carleson operators swiftly became a subject of intense interest, with work by Hunt $[\mathrm{Hu}]$ and Sjölin $[\mathrm{Sj}]$ that extended Carleson's result to other $L^{p}$ spaces and to higher dimensions, and quite different approaches of Fefferman [Fe4] and Lacey and Thiele [LT], which stimulated the development of time-frequency analysis. In total, this work established a theorem of the following general form: define for each $\lambda \in \mathbb{R}^{n}$ the operator

$$
T_{\lambda} f(x)=\int_{\mathbb{R}^{n}} f(x-y) e^{i \lambda \cdot y} K(y) d y
$$

for a kernel $K$ belonging to an appropriate class of Calderón-Zygmund kernels. Then the Carleson operator $f \mapsto \sup _{\lambda \in \mathbb{R}^{n}}\left|T_{\lambda} f(x)\right|$ is a bounded operator on $L^{p}\left(\mathbb{R}^{n}\right)$ for all $1<p<\infty$, and all $n \geq 1$.

II.6.1. Polynomial Carleson operators and $T T^{*}$ methods. Stein initiated the study of polynomial Carleson operators, in which the linear phase $\lambda \cdot y$ in (II.6.1) is replaced by a polynomial of higher degree [169], [191]. Precisely, given any polynomial $P$ in the set $\mathcal{P}_{d}$ of all real-valued degree $d$ polynomials on $\mathbb{R}^{n}$, define

$$
T_{P} f(x)=\int_{\mathbb{R}^{n}} f(x-y) e^{i P(y)} K(y) d y .
$$

Stein's influential question was whether for each $1<p<\infty$ there exists a constant $A=A(p, n, d)$ such that for all $f \in L^{p}\left(\mathbb{R}^{n}\right)$,

$$
\left\|\sup _{P \in \mathcal{P}_{d}}\left|T_{P} f(x)\right|\right\|_{L^{p}\left(\mathbb{R}^{n}\right)} \leq A\|f\|_{L^{p}\left(\mathbb{R}^{n}\right)} .
$$

In [169] Stein proved the first result in this direction on $\mathbb{R}^{1}$, in which the supremum was allowed to vary over purely quadratic phase polynomials $P(y)=\lambda y^{2}, \lambda \in \mathbb{R}$. This work used an asymptotic for the Fourier transform of the kernel $e^{i \lambda y^{2}} / y$, not easily adapted to higher-degree polynomials or higher dimensions. But then in 2001 Stein and Wainger [191] uncovered a simple, clever argument in a special case: if the supremum in (II.6.3) is restricted to the class $\mathcal{P}_{d}^{*}$ of degree $d$ polynomials without any linear terms, $T T^{*}$ methods can prove $L^{p}$ bounds $(1<p<\infty)$ for the operator $f \mapsto \sup _{P \in \mathcal{P}_{d}^{*}}\left|T_{P} f(x)\right|$, for any degree $d \geq 2$ and in any dimension $n \geq 1$.

The essential model problem is the following $L^{2}$ question: given any measurable stopping-time function $\lambda(x): x \mapsto\left(\lambda_{\alpha}\right)_{\alpha}$ yielding a corresponding phase polynomial $P_{\lambda(x)}(y)=\sum_{2 \leq|\alpha| \leq d} \lambda_{\alpha} y^{\alpha}$, prove that as long as the coefficient values are large in total, say $\sum_{\alpha}\left|\bar{\lambda}_{\alpha}\right| \approx r \geq 1$, then there exists $\delta_{0}>0$ independent of the stoppingtime function such that

$$
\left\|\int_{\mathbb{R}^{n}} f(x-y) e^{i P_{\lambda(x)}(y)} \eta(y) d y\right\|_{L^{2}\left(\mathbb{R}^{n}(d x)\right)} \leq r^{-\delta_{0}} A\|f\|_{L^{2}\left(\mathbb{R}^{n}\right)}
$$

for all $r \geq 1$. Here $\eta$ is a smooth, compactly supported bump function. To treat this by $T T^{*}$ methods, one studies the operator

$$
T T^{*} f(x)=\int_{\mathbb{R}^{n}} f(x-y) \Phi^{\lambda(x), \lambda(x-y)}(y) d y,
$$


in which

$$
\Phi^{\lambda(x), \lambda(x-y)}(y)=\int_{\mathbb{R}^{n}} e^{i\left(P_{\lambda(x)}(y+z)-P_{\lambda(x-y)}(z)\right)} \eta(y+z) \bar{\eta}(z) d z .
$$

A van der Corput estimate with respect to $z$ provides a bound with decay like $r^{-\delta_{0}}$ if the coefficients of $P_{\lambda(x)}(y+z)-P_{\lambda(x-y)}(z)$ are $\gtrsim r$, but this is hard to detect due to possible interactions of the stopping times $\lambda(x)$ and $\lambda(x-y)$. The key insight of Stein and Wainger's argument is that within the restricted class $\mathcal{P}_{d}^{*}$, terms in $P_{\lambda(x)}(y+z)-P_{\lambda(x-y)}(z)$ that are linear in $z$ arise only from $P_{\lambda(x)}(y+z)$, thus eliminating the possibility of such interactions, and allowing van der Corput methods to succeed in proving (II.6.4).

A few years later, Victor Lie resolved the general problem for the full class $\mathcal{P}_{d}$ in one dimension, in a tour-de-force work via methods of time-frequency analysis [Li1]. Recently, both Lie [Li2] and Pavel Zorin-Kranich [Z0] have resolved the $L^{p_{-}}$ boundedness $(1<p<\infty)$ for the polynomial Carleson operator for $\mathcal{P}_{d}$ for any $d \geq 2$, in any dimension $n \geq 1$.

II.6.2. Polynomial Carleson operators and curvature. A second direction Stein inspired in the study of polynomial Carleson operators related to his longstanding interest in the role of curvature in Radon-type behavior of singular integrals and maximal functions - an interest dating back, for example, to another influential work with Wainger [94]. Looking at a notebook I kept in graduate school, I see that on a day in March 2007 Stein posed the following question to me. Define an operator integrating along a paraboloid, initially acting on functions $f$ of Schwartz class, by

$$
R_{P} f(x, t)=\int_{\mathbb{R}^{n}} f\left(x-y, t-|y|^{2}\right) e^{i P(y)} K(y) d y,
$$

where $P$ is a real-valued polynomial of degree $d$ and $K$ is a Calderón-Zygmund kernel. For what class of polynomials $\mathcal{P}_{d}^{\sharp}$ can you prove that for each $1<p<\infty$ an a priori estimate holds,

$$
\left\|\sup _{P \in \mathcal{P}_{d}^{\sharp}}\left|R_{P} f\right|\right\|_{L^{p}\left(\mathbb{R}^{n+1}\right)} \leq A\|f\|_{L^{p}\left(\mathbb{R}^{n+1}\right)} ?
$$

In fact, it is reasonable to think this should be true for the full class $\mathcal{P}_{d}$, but Stein's suggestion was to apply $T T^{*}$ methods in a restricted class $\mathcal{P}_{d}^{\sharp}$, sufficiently distinguished from the defining function $\left(t,|t|^{2}\right)$ of the paraboloid. This was particularly appealing because it was a completely new type of question, but had an inherently classical flavor.

Po-Lam Yung and I took up this project after we wrote theses with Stein on other topics. We proved that for dimensions $n \geq 2$, (II.6.5) holds for a class $\mathcal{P}_{d}^{\sharp}$ defined by any linear combinations of fixed homogeneous polynomials $p_{j}(y)$ of degrees $j=2, \ldots, d$, as long as $p_{2}(y)$ is not a multiple of $|y|^{2}$. Our approach in [PY] was heavily influenced by many ideas Stein promoted either through his work or exposition, including Littlewood-Paley theory, square function methods for bounding the Hilbert transform on the parabola, and van der Corput estimates. Later we returned to the problem of monomial curves in the plane via other methods, in GPRY.

Stein's questions about polynomial Carleson operators, and their interaction with Radon-type behavior, have exposed territory that is rich with open questions, 
and with connections to current developments in harmonic analysis. For example, to mention just one other recent paper (among many), Shaoming Guo, Joris Roos and Yung's work [GRY] on sharp variation-norm results for operators of the form (II.6.2) has revealed connections to local smoothing estimates and square function estimates for linear Schrödinger equations, related to Stein's restriction conjecture, and decoupling. Open problems stemming from Stein's initial questions on polynomial Carleson operators will likely resonate for years to come.

\section{II.7. OSCILLATORY INTEGRALS AND THE ROLE OF CURVATURE}

contributed by C. Sogge

I was very fortunate to be a student of Elias Stein during a time when he was proving a number of pioneering results that highlighted the important role that curvature plays in harmonic analysis. This theme has continued to this day and has led to a number of major results in areas such as linear and nonlinear partial differential equations, number theory, and, of course, Fourier analysis.

Let me focus on three of Stein's results from this period. The first two, his spherical maximal theorem and his work on $L^{2}$ restriction theorems for the Fourier transform, culminating in the Stein-Tomas restriction theorem, were proved a few years before I became his student. Both of these results have been hugely influential, and they are remarkable since there is no a priori reason that they should be valid since they involve integrals over spheres of functions that are only defined almost everywhere. The third result, which set an important bar and has had many applications, was originally presented in a course that I took during that period. This is Stein's oscillatory integral theorem in higher dimensions, which actually implies the Stein-Tomas restriction theorem and extends earlier two-dimensional results of Carleson-Sjölin, Fefferman and Hörmander.

Stein's remarkable spherical maximal theorem [83] says that in dimensions $n \geq 3$ if $p>n /(n-1)$, not only can one make sense of the spherical averages of $f \in L^{p}\left(\mathbb{R}^{n}\right)$,

$$
A_{t} f(x)=\int_{S^{n-1}} f(x-t y) d S(y),
$$

but the associated maximal function $A^{*} f(x)=\sup _{t>0}\left|A_{t} f(x)\right|$ is actually bounded on $L^{p}\left(\mathbb{R}^{n}\right)$. Specifically, for each $p>n /(n-1)$, he showed that one has the uniform bounds

$$
\left\|A^{*} f\right\|_{L^{p}\left(\mathbb{R}^{n}\right)} \leq C_{p}\|f\|_{L^{p}\left(\mathbb{R}^{n}\right)}, \quad \text { if } f \in \mathcal{S}\left(\mathbb{R}^{n}\right),
$$

and he also showed that the range of exponents is sharp. Since the space of Schwartz class functions is dense in $L^{p}\left(\mathbb{R}^{n}\right)$, this allows one to define $A^{*} f$ whenever $f \in L^{p}\left(\mathbb{R}^{n}\right)$ and extend the maximal inequality (I.1.1) to such functions, which is remarkable as they are only defined almost everywhere.

What makes this possible is the curvature of the sphere. If $S^{n-1}$, for instance, were replaced by a hypersurface which contained a piece of a hyperplane, then no such result would be possible. Stein's proof of (I.1.1) uses the fact that the Fourier transform, $\widehat{d S}(\xi)$, of surface measure on $S^{n-1}$ decays. Stein proved his spherical maximal function by showing that when $n \geq 3$ a stronger result holds for $L^{2}\left(\mathbb{R}^{n}\right)$ which leads to (II.7.1) via an interpolation argument that uses the boundedness of the Hardy-Littlewood maximal operator near $L^{1}$. This argument does not work when $n=2$ since, as Stein pointed out, the circular maximal operator 
is not bounded on $L^{2}\left(\mathbb{R}^{2}\right)$. Bourgain [Bo1] later extended Stein's result to the twodimensional case by showing that (II.7.1) also holds for all $p>2$ when $n=2$.

In a collaboration starting when I was his graduate student, Stein and I extended his results in a series of papers, [129], [136], and [148], to variable coefficient maximal theorems, which also allowed smoothly varying surfaces whose principal curvatures were allowed to vanish to finite order. In this work we naturally started to develop new techniques to study $L^{p}$-bounds for Fourier integral operators, and sharp estimates for these operators were later obtained in our joint work with Seeger [153].

Motivated by all of this and also by Stein's work with Phong [132] on the role of what they called rotational curvature, I was able to extend Bourgain's circular maximal theorem to the variable coefficient setting in [So2] by writing down the first local smoothing estimates for Fourier integral operators, although the ones which are related to the circular maximal operator were implicit in Bourgain's work Bo1. I also formulated a local smoothing conjecture for certain families of Fourier integral operators that was inspired by my work with Stein as well as his oscillatory integral theorem. Over the years there has been much work on this, including the development of the decoupling method of Bourgain, Demeter, and Wolff that has lead to an explosion of activity in harmonic analysis. All of these results trace back to Stein's beautiful spherical maximal theorem.

Stein also had a profound impact through his work on restriction phenomena for the Fourier transform. For these problems, one wishes to study the restrictions of the Fourier transforms $\hat{f}$ of $L^{p}\left(\mathbb{R}^{n}\right)$ functions $f$, for certain $1<p<2$ to hypersurfaces such as the sphere. As above, this seems a tall task since $\hat{f}$ is only defined almost everywhere; however, certain restriction bounds are possible if one restricts $\hat{f}$ to curved surfaces such as $S^{n-1}$. Thus, for instance, the $L^{2}\left(S^{n-1}\right)$ restriction problem would be to show that for a given $n \geq 2$ there are certain $1<p<2$ for which one has uniform bounds of the form

$$
\int_{S^{n-1}}|\hat{f}(\xi)|^{2} d S(\xi) \leq C_{p}\|f\|_{L^{p}\left(\mathbb{R}^{n}\right)}^{2}, \quad f \in \mathcal{S}\left(\mathbb{R}^{n}\right)
$$

Stein was the first one to observe that such inequalities are possible. In an unpublished work, he showed that (II.7.2) holds when $n=2$ and $1 \leq p \leq 8 / 7$, once again by exploiting the decay of $\widehat{d S}$. In his thesis [Fe1] with Stein, Fefferman made significant progress on this problem by showing what turned out to be near optimal results for this problem when $n=2$. The full resolution to this problem is the Stein-Tomas restriction theorem in [Tm2, stating that (II.7.2) holds for all $1 \leq p \leq 2(n+1) /(n-1)$. Simple counterexamples show that this result is optimal; however, when $n \geq 3$ an important problem, which remains open, is to show that when $f \in L^{p}\left(\mathbb{R}^{n}\right)$ and $p<2 n /(n+1), \hat{f}$ restricts to $S^{n-1}$ as a function in a certain Lebesgue space.

Stein's work on restriction problems and his introduction of this problem to the subject has had an enormous impact on harmonic analysis, PDEs, and geometric analysis. Fourier restriction estimates and related bounds have become an indispensable tool for researchers in nonlinear dispersive equations, for instance. Also, my work on eigenfunction theory on Riemannian manifolds [So1] is an extension of the Stein-Tomas restriction theorem to this setting. 
I was able to prove eigenfunction bounds using Stein's oscillatory integral theorem [131]. Stein's oscillatory integral bounds also inspired work on local smoothing estimates. In a complementary work to [131], Bourgain [Bo8 provided counterexamples showing that Stein's oscillatory integral theorem is optimal in odd dimensions. This, together with Stein's positive result, opened up another set of important problems in which curvature plays a key role.

Stein was a very broad mathematician whose research opened up many new avenues and provided us with many key tools over the years. He was also a very gifted expositor and teacher, as well as a very generous mentor. He was always a role model that most of us could only strive to emulate.

\section{II.8. Multiparameter SINGUlar RADON TRANSFORMS}

contributed by B. Street

Eli Stein played a central role in the development of singular Radon transforms; these are operators of the form

$$
T f(x)=\psi(x) \int f(\gamma(t, x)) K(t) d t,
$$

where $\gamma(t, x): \mathbb{R}_{0}^{N} \times \mathbb{R}_{0}^{n} \rightarrow \mathbb{R}^{n}$ is a $C^{\infty}$-function defined on a neighborhood of the origin in $(t, x) \in \mathbb{R}^{N} \times \mathbb{R}^{n}$ and satisfies $\gamma(0, x)=x, \psi \in C_{0}^{\infty}\left(\mathbb{R}^{n}\right)$ is supported near $x=0$ and that $K(t)$ is a singular kernel supported near $t=0$. A great deal of work, by many authors, has been devoted to understanding such operators when $K(t)$ is a Calderón-Zygmund kernel; these are kernels satisfying

$$
\left|\partial_{t}^{\alpha} K(t)\right| \leq C_{\alpha}|t|^{-N-|\alpha|}, \quad \forall \alpha \in \mathbb{N}^{N},
$$

along with a certain cancellation condition. The history of these operators is described in more detail in other sections in this article, but much of this work culminated in the influential article of Christ, Nagel, Stein, and Wainger [182]. They gave sufficient conditions on $\gamma$ under which the operator (II.8.1) is bounded on $L^{p}(1<p<\infty)$ for every Calderón-Zygmund kernel $K$, supported near $t=0$. More recently, Stein and I strengthened these results and extended them to the multiparameter setting [210], SSt2, [213], [215].

This includes the situation when $K(t)$ is a product kernel supported near $t=0$. To define product kernels, we decompose $t \in \mathbb{R}^{N}$ into $\nu$ factors $\left(t_{1}, \ldots, t_{\nu}\right) \in \mathbb{R}^{N}=$ $\mathbb{R}^{N_{1}} \times \cdots \times \mathbb{R}^{N_{\nu}}$. A product kernel satisfies estimates like

$$
\left|\partial_{t_{1}}^{\alpha_{1}} \cdots \partial_{t_{\nu}}^{\alpha_{\nu}} K\left(t_{1}, \ldots, t_{\nu}\right)\right| \leq C_{\alpha_{1}, \ldots, \alpha_{\nu}}\left|t_{1}\right|^{-N_{1}-\left|\alpha_{1}\right|} \ldots\left|t_{\nu}\right|^{-N_{\nu}-\left|\alpha_{\nu}\right|}
$$

again, along with a certain cancellation condition. These papers used several ideas that Stein championed in his other works as well: use of Littlewood-Paley theory to study operators at different scales, approximation of operators by nilpotent Lie groups, using an underlying non-Euclidean geometry adapted to the operators, and generalized notions of scaling.

We begin by briefly describing the work of Christ, Nagel, Stein, and Wainger [182]. Write $\gamma_{t}(x)=\gamma(t, x)$. Since $\gamma_{0}(x)=x$, for $t$ sufficiently small, $\gamma_{t}(\cdot)$ is a diffeomorphism onto its image; we let $\gamma_{t}^{-1}$ denote the inverse mapping. Let $W(t, x):=\left.\frac{d}{d \epsilon}\right|_{\epsilon=1} \gamma_{\epsilon t} \circ \gamma_{t}^{-1}(x) \in T_{x} \mathbb{R}^{n}$, so that $W(t)$ is a vector field depending smoothly on $t$, satisfying $W(0)=0$. We expand $W(t)$ into a Taylor series in the $t$ variable: $W(t) \sim \sum t^{\alpha} X_{\alpha}$, where $X_{\alpha}$ is a smooth vector field defined on a neighborhood of the origin in $\mathbb{R}^{n}$. 
Theorem II.8.1 ([182]). Suppose that $\left\{X_{\alpha}: \alpha \in \mathbb{N}^{N}\right\}$ satisfies Hörmander's condition near $0 \in \mathbb{R}^{n}$, i.e., that the Lie algebra generated by $\left\{X_{\alpha}\right\}$ spans the tangent space at every point near 0 . Then, any operator of the form (II.8.1), where $K(t)$ is a Calderón-Zygmund kernel, extends to a bounded operator $L^{p} \rightarrow L^{p}$, $1<p<\infty$.

Because of the assumption that $\left\{X_{\alpha}: \alpha \in \mathbb{N}^{\nu}\right\}$ satisfies Hörmander's condition, there is a natural Carnot-Carathéodory metric adapted to $\gamma$. Nagel, Stein, and Wainger [128] gave a detailed quantitative study of this metric and in particular showed that this metric, when paired with Lebesgue measure, gives rise to a space of homogeneous type. This is a key component when understanding the LittlewoodPaley theory adapted to the operators in Theorem II.8.1.

In [St2] and [215] we moved beyond Hörmander's condition in the following way. Suppose the $C^{\infty}$-module generated by the Lie algebra generated by $\left\{X_{\alpha}: \alpha \in \mathbb{N}^{\nu}\right\}$ is finitely generated as a $C^{\infty}$-module 9 Then the classical Frobenius theorem foliates the ambient space into leaves and $\left\{X_{\alpha}: \alpha \in \mathbb{N}^{N}\right\}$ satisfies Hörmander's condition on each leaf. Provided $\gamma_{t}(x)$ lies in the leaf passing through $x$, for each $t$, one might hope to prove an analogue of Theorem II.8.1 Indeed this is possible under a natural, though technical, quantitative version of the assumption that $\gamma_{t}(x)$ lies in the leaf passing through $x$, for each $t$. This required a new, quantitative version of the Frobenius theorem which was established in [St1, building on [128] and TW].

Inspired by work of Bourgain [Bo6], we showed in [213] that all of these hypothesis on $\gamma$ are automatically satisfied when $\gamma(t, x)$ is real analytic in both variables; this is closely related to the classical fact that germs of real analytic functions form a Noetherian ring.

Theorem II.8.2 (Stein and Street [213]). Suppose $\gamma(t, x)$ is a real analytic function defined near $(0,0) \in \mathbb{R}^{N} \times \mathbb{R}^{n}$ and satisfying $\gamma(0, x)=x$. Then for every CalderónZygmund kernel $K(t)$ supported near $0 \in \mathbb{R}^{N}$, the operator given by (II.8.1) extends to a bounded operator on $L^{p}, 1<p<\infty$.

Theorem II.8.2 can be interpreted as saying that there is no algebraic obstruction to the $L^{p}$-boundedness of operators of the form (II.8.1), when $K(t)$ is a CalderónZygmund kernel. It is known that when $\gamma$ is $C^{\infty}$, these operators need not be bounded on any $L^{p}$, but this requires the use of functions which vanish to infinite order.

We now turn to the multiparameter setting, when $K(t)$ is a product kernel. Unlike the single-parameter setting, there are algebraic obstructions to the boundedness of the operators under consideration, even when $\gamma(t, x)$ is a polynomial. This was first observed by Nagel and Wainger [NW]. With $n=1, N=2$,

$$
f \mapsto \psi(x) \int f(x-s t) \frac{\eta(s) \eta(t)}{s t} d s d t
$$

is not bounded on $L^{2}$ if $\eta, \psi \in C_{0}^{\infty}(\mathbb{R})$ are nonzero at 0 .

The assumptions in [St2], [215] are separated into two parts, both of which are somewhat technical. The first part is a finite type assumption; namely a scale-invariant, multiparameter version of the assumptions discussed above concerning the Frobenius theorem. The finite type assumption is automatic when

\footnotetext{
${ }^{9}$ Under the hypothesis of Hörmander's condition, this module is finitely generated: it is generated by the coordinate vector fields.
} 
$\gamma$ is real analytic. The second assumption is an algebraic assumption, made to avoid examples like (II.8.2). In this assumption, we consider the power series $W\left(t_{1}, \ldots, t_{\nu}\right) \sim \sum t_{1}^{\alpha_{1}} \cdots t_{\nu}^{\alpha_{\nu}} X_{\alpha}$ and we separate the vector fields into two sets: those $X_{\alpha}$ where $\alpha=\left(\alpha_{1}, \ldots, \alpha_{\nu}\right)$ has only one $\alpha_{\mu} \neq 0$ (the pure powers), and the rest of the $X_{\alpha}$ (the nonpure powers). The algebraic assumption is that the vector fields corresponding to the nonpure powers are spanned by the Lie algebra generated by the vector fields corresponding to the pure powers in a quantitative, scale-invariant way. Under these two assumptions, which are described in detail in [St2], [213], [215], it is shown that the operator given by (II.8.1), when $K\left(t_{1}, \ldots, t_{\nu}\right)$ is a product kernel, extends to a bounded operator on $L^{p}, 1<p<\infty$.

We also study corresponding maximal functions under similar hypotheses. In particular, inspired by work of Christ [Ch7, we showed that the corresponding maximal functions are always bounded when $\gamma$ is real analytic:

Theorem II.8.3 (Stein and Street [213]). Let $\gamma(t, x)$ be a real analytic function defined near $(0,0) \in \mathbb{R}^{N} \times \mathbb{R}^{n}$ satisfying $\gamma(0, x)=x$, and let $\psi \in C_{0}^{\infty}\left(\mathbb{R}^{n}\right)$ be supported near $x=0 \in \mathbb{R}^{n}$ and nonnegative. Then the operator

$$
f \mapsto \sup _{0<\delta_{1}, \ldots, \delta_{N} \ll 1} \psi(x) \int_{|t|<1}\left|f\left(\gamma_{\delta_{1} t_{1}, \ldots, \delta_{N} t_{N}}(x)\right)\right| d t
$$

is bounded on $L^{p}, 1<p \leq \infty$.

Working with and learning from Eli was one of the highlights of my education. His excitement for new ideas, both big and small, was infectious and encouraging. His guidance on which questions were important, interesting, and within reach was invaluable. He was incredibly generous and helped many young mathematicians (including me) get started on their careers. His kindness shaped the harmonic analysis community, making it less competitive and more collaborative. It will not be the same without him.

\section{II.9. The RESTRICTION CONJECTURE}

contributed by T. Tao

If $f \in L^{1}\left(\mathbb{R}^{n}\right)$ is an absolutely integrable function on a Euclidean space $\mathbb{R}^{n}$, then by the dominated convergence theorem, the Fourier transform

$$
\hat{f}(\xi):=\int_{\mathbb{R}^{n}} f(x) e^{-2 \pi i x \cdot \xi} d x
$$

is a continuous function on $\mathbb{R}^{n}$; in particular, it can be meaningfully restricted to any subset $S$ of $\mathbb{R}^{n}$ without difficulty. On the other hand, if $f \in L^{2}\left(\mathbb{R}^{n}\right)$ is merely assumed to be a square-integrable function, then by the Plancherel theorem, the Fourier transform of $f$ is an arbitrary $L^{2}\left(\mathbb{R}^{n}\right)$ function, and in particular does not in general have a meaningful restriction to any measure zero subset $S$ of $\mathbb{R}^{n}$.

Nevertheless, in the late 1960s, Stein observed [Fe1] that for certain measure zero sets $S$ exhibiting nontrivial curvature, such as the unit sphere $S^{n-1}$, it was still possible to restrict the Fourier transform $\hat{f}$ of functions $f$ in $L^{p}\left(\mathbb{R}^{n}\right)$ if $1 \leq p<2$ was sufficiently small. In particular, he established the first example of what we now call a restriction theorem: 
Theorem II.9.1 (Stein's first restriction theorem [Fe1]). Let $n \geq 2$. For any test function $f \in C_{c}^{\infty}\left(\mathbb{R}^{n}\right)$, and any $1 \leq p<\frac{4 n}{3 n+1}$, one has the restriction estimate

$$
\|\hat{f}\|_{L^{2}\left(S^{n-1}, d \sigma\right)} \leq C_{n, p}\|f\|_{L^{p}\left(\mathbb{R}^{n}\right)},
$$

where $d \sigma$ is surface measure on the unit sphere $S^{n-1}$, and $C_{n, p}$ is a constant depending only on $n, p$.

As a corollary of this and the density of $C_{c}^{\infty}\left(\mathbb{R}^{n}\right)$ in $L^{p}\left(\mathbb{R}^{n}\right)$, one can define a square-integrable restriction of $\hat{f}$ to the sphere for any $f \in L^{p}\left(\mathbb{R}^{n}\right)$. The proof (see section (1.2) is very simple: basic Fourier analysis yields the identity

$$
\|\hat{f}\|_{L^{2}\left(S^{n-1}, d \sigma\right)}^{2}=\langle f, f * \widehat{d \sigma}\rangle .
$$

Standard asymptotics for Bessel functions show that $\widehat{d \sigma} \in L^{q}\left(\mathbb{R}^{n}\right)$ for any $q>\frac{2 n}{n-1}$. The claim then follows from Young's inequality and Hölder's inequality.

This simple argument was an early prototype of what is now a standard tool in harmonic analysis, the $T T^{*}$ method. It was soon realized that this restriction phenomenon was closely connected to the $L^{p}$-convergence of spherically summed Fourier series (or related operations, such as application of Bochner-Riesz multipliers) [Fe1, as well as to the $L^{p}$-behavior of solutions to dispersive equations such as the linear wave or Schrödinger equation $[\mathrm{Sr}$; both connections have since become absolutely fundamental in the further study of these topics. The range of exponents for which restriction phenomena were possible were also expanded upon in later work. In [Tm1,Tm2] Tomas and Stein showed that Theorem II.9.11 in fact held for all $1 \leq p \leq \frac{2(n+1)}{n+3}$, and an unpublished counterexample of Knapp (reproduced for instance in [Tm2]) shows that this range is best possible. The argument is similar to that given above, but it also exploits the oscillatory nature of the convolution kernel $\widehat{d \sigma}$, and the endpoint $p=\frac{2(n+1)}{n+3}$ requires the full power of the Stein complex interpolation theorem [1].

Stein also realized that further useful restriction estimates could be possible if one replaced the $L^{2}$-norm in (II.9.1) by other norms, leading him to formulate Stein's restriction conjecture [96]

$$
\|\hat{f}\|_{L^{q}\left(S^{n-1}, d \sigma\right)} \leq C_{n, p, q}\|f\|_{L^{p}\left(\mathbb{R}^{n}\right)}
$$

whenever $n \geq 2, f \in C_{c}^{\infty}\left(\mathbb{R}^{n}\right), 1 \leq p<\frac{2 n}{n-1}$, and $\frac{n+1}{p^{\prime}} \leq \frac{n-1}{q}$ (here $p^{\prime}$ is the dual exponent to $p$, thus $\frac{1}{p}+\frac{1}{p^{\prime}}=1$ ). The Knapp example mentioned earlier (as well as a variant of a classical counterexample of Herz $[\mathrm{Hr}$ ) show that the range of exponents here is optimal. Similar conjectures can then be made for other surfaces than the sphere $S^{n-1}$.

The restriction conjecture is still not fully resolved (except in two dimensions, by the work of Zygmund [Zy2]), but has proved to be enormously influential in harmonic analysis and partial differential equations, with many unexpected connections. For instance, the restriction estimates of Strichartz $\mathrm{Sr}$ ] for linear dispersive equations mentioned previously turned out to be the perfect tool for studying nonlinear perturbations of such equations, and they have since become as central to the study of nonlinear dispersive equations as Sobolev and Schauder estimates are to the study of nonlinear elliptic equations. The modern theory of these equations now goes beyond the Strichartz estimates and relies on many further linear, 
bilinear, and multilinear estimates for such equations, but the proofs of such estimates often still rely on the basic techniques pioneered or promoted by Stein, such as dyadic or wave packet decomposition, the $T T^{*}$ method, and exploitation of the principle of stationary phase.

As indicated previously, the conjecture is closely related to the study of BochnerRiesz multipliers; the latter were then found in [Fe2 to be linked to the Kakeya conjecture regarding the size of Besicovitch sets, and a further conjecture of Stein [96] proposes further links between the restriction conjecture and objects related to the Kakeya conjecture, such as the Kakeya maximal operator. (A formal implication of the Kakeya conjecture from the restriction conjecture was established in [Fe3], and a formal implication of the restriction conjecture from the BochnerRiesz conjecture was established in [Ta1]. The relevance of Besicovitch type sets to harmonic analysis was previously anticipated by Stein and Weiss [48]). Recent progress on the restriction conjecture has drawn in a remarkable array of tools, ranging from bilinear and multilinear estimates, to wave packet decompositions, to induction on scales, to polynomial partitioning, to (most recently) decoupling theorems; see for instance [Ta2 for further discussion of several of these topics. A recent highlight of this theory is the application by Bourgain, Demeter, and Guth [BDG] of decoupling theorems that drew heavy inspiration from the literature on the restriction conjecture in order to prove a major conjecture in analytic number theory, namely the Vinogradov main conjecture,

$$
\int_{[0,1]^{k}}\left|\sum_{n \leq N} e^{2 \pi i\left(\alpha_{1} n+\cdots+\alpha_{k} n^{k}\right)}\right|^{2 s} d \alpha_{1} \ldots d \alpha_{k} \leq C_{\epsilon, k} N^{\epsilon}\left(N^{s}+N^{2 s-\frac{k(k+1)}{2}}\right),
$$

for all $N \geq 1, k \geq 1, \epsilon>0$, and $s>0$. Such decoupling theorems have also been used to make progress on several problems in incidence combinatorics; see for instance $\mathrm{BD}$. Certainly the ongoing impact of Stein's restriction conjecture has been far broader and deeper than was first imagined!

\section{Part III. Appendix: Eli Stein's Bibliography}

[1] Interpolation of linear operators, Trans. Amer. Math. Soc. 83 (1956), 482-492.

[2] Functions of exponential type, Ann. of Math (2) 65 (1957), 582-592.

[3] Interpolation in polynomial classes and Markoff's inequality, Duke Math. J. 24 (1957), 467-476.

[4] Note on singular integrals, Proc. Amer. Math. Soc. 8 (1957), 250-254.

[5] (with G. Weiss) On the interpolation of analytic families of operators action on $H^{p}$ spaces, Tohoku Math. J. (2) 9 (1957), 318-339.

[6] (with E. H. Ostrow) A generalization of lemmas of Marcinkiewicz and Fine with applications to singular integrals, Ann. Scuola Norm. Sup. Pisa (3) 11 (1957), 117-135.

[7] A maximal function with applications to Fourier series, Ann. of Math. (2) 68 (1958), 584-603.

[8] (with G. Weiss) Fractional integrals on n-dimensional Euclidean space, J. Math. Mech. 7 (1958), 503-514.

[9] (with G. Weiss) Interpolation of operators with change of measures, Trans. Amer. Math. Soc. 87 (1958), 159-172.

[10] Localization and summability of multiple Fourier series, Acta Math. 100 (1958), 93-147.

[11] On the functions of Littlewood-Paley, Lusin, and Marcinkiewicz, Trans. Amer. Math. Soc. 88 (1958), 430-466. 
[12] (with G. Weiss) An extension of a theorem of Marcinkiewicz and some of its applications, J. Math. Mech. 8 (1959), 263-284.

[13] (with G. Weiss) On the theory of harmonic functions of several variables I. The theory of $H^{p}$ spaces, Acta Math. 103 (1960), 25-62.

[14] (with R. A. Kunze) Uniformly bounded representations and harmonic analysis of the $2 \times 2$ real unimodular group, Amer. J. Math. 82 (1960), 1-62.

[15] The characterization of functions arising as potentials, Bull. Amer. Math. Soc. 67 (1961) 102-104.

[16] On some functions of Littlewood-Paley and Zygmund, Bull. Amer. Math. Soc. 67 (1961), 99-101.

[17] On limits of sequences of operators, Ann. of Math (2) 74 (1961), 140-170.

[18] On the theory of harmonic functions of several variables II. Behavior near the boundary, Acta Math. 106 (1961), 137-174.

[19] On certain exponential sums arising in multiple Fourier series, Ann. of Math (2) 73 (1961), 87-109.

[20] (with R. A. Kunze) Analytic continuation of the principal series, Bull. Amer. Math. Soc. 67 (1961), 543-546.

[21] On the maximal ergodic theorem, Proc. Nat. Acad. Sci. U.S.A. 47 (1961), 1894-1897.

[22] (with R. A. Kunze) Uniformly bounded representations II. Analytic continuation of the principal series of representations of the $n \times n$ complex unimodular groups, Amer. J. Math. 83 (1961), 723-786.

[23] (with A. Zygmund) Smoothness and differentiability of functions, Ann. Univ. Sci. Budapest, Eötvös Sect. Math. 3-4 (1960-61), 295-307.

[24] The characterization of functions arising as potentials II, Bull. Amer. Math. Soc. 68 (1962), 577-582.

[25] Conjugate harmonic functions in several variables, Proc. Internat. Congr. Mathematicians (Stockholm, 1962) pp. 414-420, Inst. Mittag-Leffler, Djursholm (1963).

[26] (with A. Zygmund) On the differentiability of functions, Studia Math. 23 (1963/64), 248-283.

[27] (with G. Weiss and M. Weiss) $H^{p}$-classes of holomorphic functions in tube domains, Proc. Nat. Acad. Sci. U.S.A. 52 (1964), 1035-1039.

[28] (with B. Muckenhoupt) Classical expansions and their relations to conjugate functions, Trans. Amer. Math. Soc. 118 (1965), 17-92.

[29] Note on the boundary of holomorphic functions, Ann. of Math. (2) 82 (1965), 351-353.

[30] (with S. Wainger) Analytic properties of expansions and some variants of Parseval-Plancherel formulas, Ark. Mat. 5 (1965), 553-567.

[31] (with A. Zygmund) On the fractional differentiability of functions, London Math. Soc. (3) 14A (1965), 249-264.

[32] A survey of representations of non-compact groups, 1965 Lect. Sem. on HighEnergy Physics and Elementary Particles (Trieste, 1965) pp. 563-584, International Atomic Energy Agency, Vienna.

[33] Classes $H^{p}$, multiplicateurs, et fonctions de Littlewood-Paley (French), C. R. Acad. Sci. Paris Sér. A-B 263 (1966), A716-A719.

[34] Classes $H^{p}$, multiplicateurs et fonctions de Littlewood-Paley. Applications de résultats antérieurs (French), C. R. Acad. Sci. Paris Sér. A-B 263 (1966), A780-A781.

[35] (with R. Kunze) Uniformly bounded representations III. Intertwining operators, Amer. J. Math. 89 (1967), 385-442.

[36] Singular integrals, harmonic functions and differentiability properties of functions of several variables, Proc. Sympos. Pure Math. 1966, 316-335, Amer. Math. Soc., Providence, R.I., 1967. 
[37] Classes $H^{p}$ et multiplicateurs: Cas n-dimensionnel (French), C. R. Acad. Sci. Paris Sér. A-B 264 (1967), A107-A108.

[38] Analysis in matrix spaces and some new representations of $S L(N, C)$, Ann. of Math. (2) 86 (1967), 461-490.

[39] (with A. Zygmund) Boundedness of translation invariant operators on Hölder spaces and $L^{p}$ spaces, Ann. of Math. (2) 85 (1967), 337-349.

[40] The analogues of Fatou's theorem and estimates for maximal functions, 1968 Geometry of Homogeneous Bounded Domains (C.I.M.E., 3 Ciclo, Urbino, 1967) pp. 291-307, Rome.

[41] (with A. Zygmund) On the boundary behavior of harmonic functions, 1968 Orthogonal Expansions and their Continuous Analogues (Proc. Conf., Edwardsville, Ill., 1967) pp. 127-141 Southern Illinois Univ. Press, Carbondale, Ill.

[42] (with A. Korányi) Fatou's theorem for generalized halfplanes, Ann. Scuola Norm. Sup. Pisa Cl. Sci. (3) 22 (1968), 107-112.

[43] (with G. Weiss) Generalizations of the Cauchy-Riemann equations and representations of the rotation group, Amer. J. Math. 90 (1968), 163-196.

[44] (with A. Grossman and G. Loupias) An algebra of pseudodifferential operators and quantum mechanics in phase space, Ann. Inst. Fourier (Grenoble) 18 (1968), 343-368.

[45] (with N. J. Weiss) Convergence of Poisson integrals for bounded symmetric domains, Proc. Nat. Acad. Sci. U.S.A. 60 (1968), 1160-1162.

[46] Note on the class L $\log L$, Studia Mathematica, 32 (1969), 305-310.

[47] (with A. W. Knapp) Singular integrals and the principal series, Proc. Nat. Acad. Sci. U.S.A. 63 (1969), 281-284.

[48] (with N. J. Weiss) On the convergence of Poisson integrals, Trans. Amer. Math. Soc. 140 (1969), 35-54.

[49] Singular integrals and differentiability properties of functions, Princeton Mathematical Series No. 30, Princeton Univ. Press 1970, xiv+290 pages.

[50] (with A. W. Knapp) Singular integrals and the principal series II, Proc. Nat. Acad. Sci. U.S.A. 66 (1970), 13-17.

[51] (with A. W. Knapp) The existence of complementary series, Problems in analysis (Papers dedicated to Salomon Bochner, 1969), pp. 249-259. Princeton Univ. Press, Princeton, N.J., 1970.

[52] Topics in harmonic analysis related to the Littlewood-Paley theory, Annals of Mathematics Studies 63, Princeton University Press, Princeton, N.J.; Univ. Tokyo Press, Tokyo 1970 viii+146 pp.

[53] Analytic continuation of group representations, Advances in Math. 4 (1970), 172-207.

[54] Boundary values of holomorphic functions, Bull. Amer. Math. Soc. 76 (1970), 1292-1296.

[55] Variations on the Littlewood-Paley theme, 1970 Lectures in Modern Analysis and Applications, III pp. 1-17 Lecture Notes in Mathematics, Vol. 170. Springer, Berlin.

[56] (with S. Wainger) The estimating of an integral arising in multiplier transformations, Studia Math. 35 (1970), 101-104.

[57] (with G. Weiss) Introduction to Fourier analysis on Euclidean spaces, Princeton Mathematical Series No. 32. Princeton University Press, Princeton, N.J., 1971, $\mathrm{x}+297 \mathrm{pp}$.

[58] Analytic continuation of group representations, A James K. Whittemore Lecture in Mathematics given at Yale University, 1967, Yale Mathematical Monographs 2, Yale University Press, New Haven, Conn.-London, 1971, iv+36 pp.

[59] (with A. Knapp) Intertwining operators for semisimple groups, Ann. of Math. (2) 93 (1971), 489-578. 
[60] (with C. Fefferman) Some maximal inequalities, Amer. J. Math. 93 (1971), 107-115.

[61] $L^{p}$ boundedness of certain convolution operators, Bull. Amer. Math. Soc. 77 (1971), 404-405.

[62] Some problems in harmonic analysis suggested by symmetric spaces and semisimple groups, Actes du Congrès International des Mathématiciens (Nice, 1970), Tome 1, pp. 173-189. Gauthier-Villars, Paris, 1971.

[63] Boundary behavior of holomorphic functions of several complex variables, Mathematical Notes No. 11. Princeton University Press, Princeton, N.J.; Univ. Tokyo Press, Tokyo, 1972, x+72 pp.

[64] (with A. Knapp) Irreducibility theorems for the principal series, Conference on Harmonic Analysis (Maryland), pp. 197-214, Lecture Notes in Math. Vol. 266, Springer, Berlin, 1972.

[65] (with A. Korányi) $H^{2}$ spaces of generalized half-planes, Studia Math. 44 (1972), 379-388.

[66] (with C. Fefferman) $H^{p}$ spaces of several variables, Acta Math. 129 (1972), 137-193.

[67] Singular integrals and estimates for the Cauchy-Riemann equations, Bull. Amer. Math. Soc. 79 (1973), 440-445.

[68] Singular integrals related to nilpotent groups and $\bar{\partial}$-estimates, Harmonic analysis on homogeneous spaces (Proc. Sympos. Pure Math., Vol. XXVI, Williams Coll., Williamstown, Mass., 1972), pp. 363-367. Amer. Math. Soc., Providence, R.I., 1973.

[69] (with R. Kunze) Uniformly bounded representations IV. Analytic continuation of the principal series for complex classical groups of types $B_{n}, C_{n}, D_{n}$, Advances in Math. 11 (1973), 1-71.

[70] (with G. B. Folland) Parametrices and estimates for the $\bar{\partial}_{b}$ complex on strongly pseudoconvex boundaries, Bull. Amer. Math. Soc. 80 (1974), 253-258.

[71] (with J. L. Clerc) $L^{p}$ multipliers for non-compact symmetric spaces, Proc. Nat. Acad. Sci. U.S.A. 71 (1974), 3911-3912.

[72] (with A. Knapp) Singular integrals and the principal series III, Proc. Nat. Acad. Sci. U.S.A. 71 (1974), 4622-4624.

[73] (with G. B. Folland) Estimates for the $\bar{\partial}_{b}$ complex and analysis on the Heisenberg group, Comm. Pure Appl. Math. 27 (1974), 429-522.

[74] Singular integrals, old and new, in Colloquium Lectures of the 79th Summer Meeting of the American Mathematical Society, August 18-22 1975, American Mathematical Society (1975).

[75] (with P. Greiner and J. Kohn) Necessary and sufficient conditions for the solvability of the Lewy equation, Proc. Nat. Acad. Sci. U.S.A. 72 (1975), no. 9, 3287-3289.

[76] (with A. Knapp) Singular integrals and the principal series. IV, Proc. Nat. Acad. Sci. U.S.A. 72 (1975), 2459-2461.

[77] Singular integral operators and nilpotent groups, in Proceedings of C.I.M.E., Differential Operators on Manifolds, Rome, Cremonese (1975), 148-206.

[78] (with P. Greiner) A parametrix for the $\bar{\partial}-$ Neumann problem, Recontre sur l'analyse complexe à plusieurs variables et les systèmes surdéterminés, pp. 49-63. Presses Univ. Montréal, 1975.

[79] Harmonic Analysis on $\mathbb{R}^{n}$, Studies in harmonic analysis (Proc. Conf., DePaul Univ., Chicago, Ill., 1974), pp. 97-135. MAA Stud. Math. Vol. 13, Math. Assoc. Amer., Washington, D.C., 1976.

[80] (with L. P. Rothschild) Hypoelliptic differential operators and nilpotent groups, Acta Math. 137 (1976), 247-320. 
[81] (with C. Fefferman and J.-P. Kahane) The scientific achievements of Antoni Zygmund (Polish), Wiadom. Mat. (2) 19 (1976), no. 2, 91-126.

[82] (with A. W. Knapp) Intertwining operators for $S L(n, R)$, Studies in Mathematical Physics, edited by E. Lieb, B. Simon and A. Wightman, Princeton University Press (1976), 239-267.

[83] Maximal functions: Spherical means, Proc. Nat. Acad. Sci. U.S.A. 73 (1976), 2174-2175.

[84] Maximal functions: Homogeneous curves, Proc. Nat. Acad. Sci. U.S.A. 73 (1976), 2176-2177.

[85] Maximal functions: Poisson integrals on symmetric spaces, Proc. Nat. Acad. Sci. U.S.A. 73 (1976), 2547-2549.

[86] (with S. Wainger) Maximal functions associated to smooth curves, Proc. Nat. Acad. Sci. U.S.A. 73 (1976), 4295-4296.

[87] (with P. Greiner) Estimates for the $\bar{\partial}$-Neumann problem, Mathematical Notes No. 19, Princeton University Press, Princeton, N.J., 1977. iv+195 pp.

[88] (with D. H. Phong) Estimates for the Bergman and Szegö projections on strongly pseudo-convex domains, Duke Math. J. 44 (1977), no. 3, 695-704.

[89] (with N. Kerzman) The Szegö kernels in terms of Cauchy-Fantappiè kernels, Duke Math. J. 45 (1978), 197-224.

[90] (with N. Kerzman) The Cauchy kernels, the Szegö kernel and the Riemann mapping function, Math. Ann. 236 (1978), no. 1, 85-93.

[91] (with A. Nagel and S. Wainger) Differentiation in lacunary directions, Proc. Nat. Acad. Sci. U.S.A. 75 (1978), no. 3, 1060-1062.

[92] (with A. Nagel) A new class of pseudo-differential operators, Proc. Nat. Acad. Sci. U.S.A. 75 (1978), no. 2, 582-585.

[93] (with P. Greiner) On the solvability of some differential operators of the type $\square_{b}$, Several complex variables (Cortona, 1976/1977), pp. 106-165, Scuola Norm. Sup. Pisa, Pisa, 1978.

[94] (with S. Wainger) Problems in harmonic analysis related to curvature, Bull. Amer. Math. Soc. 84 (1978), no. 6, 1239-1295.

[95] (with R. Gundy) $H^{p}$ theory for the poly-disc, Proc. Nat. Acad. Sci. U.S.A. 76 (1979), no. 3, 1026-1029.

[96] Some problems in harmonic analysis, Harmonic analysis in Euclidean spaces, Part 1, pp. 3-20, Proc. Sympos. Pure Math. XXXV, Amer. Math. Soc., Providence, R.I., 1979.

[97] (with A. Nagel and S. Wainger) Hilbert transforms and maximal functions related to variable curves, Harmonic analysis in Euclidean spaces, Part 1, pp. 95-98, Proc. Sympos. Pure Math. XXXV, Amer. Math. Soc., Providence, R.I., 1979.

[98] (with A. Nagel) Some new classes of pseudo-differential operators, Harmonic analysis in Euclidean spaces, Part 2, pp. 159-169, Proc. Sympos. Pure Math. XXXV, Amer. Math. Soc., Providence, R.I., 1979.

[99] A variant of the area integral, Bull. Sci. Math. (2) 103 (1979), 449-461.

[100] (with A. Nagel) Lectures on pseudo-differential operators: Regularity theorems and applications to non-elliptic problems, Mathematical Notes No. 24, Princeton University Press, Princeton, N.J.; Univ. Tokyo Press, Tokyo, 1979, 159 pp.

[101] (with A. Knapp) Intertwining operators for semi-simple groups. II, Invent. Math. 60 (1980), 9-84.

[102] Editor's note: the differentiability of functions in $\mathbf{R}^{n}$, Ann. of Math. (2) 113 (1981), 383-385.

[103] Compositions of pseudo-differential operators, Proceedings of "Journées equation aux derivés partielles, Saint-Jean de Monts, Juin 1981", Soc. Math. de France. 
[104] (with A. Nagel and S. Wainger) Boundary behavior of functions holomorphic in domains of finite type, Proc. Nat. Acad. Sci. U.S.A. 78 (1981), no. 11, 65966599.

[105] (with A. Knapp) Some new intertwining operators for semi-simple groups, Noncommutative harmonic analysis and Lie groups (Marseille, 1980), pp. 303-336, Lecture Notes in Math. 880, Springer, Berlin-New York, 1981.

[106] (with M. H. Taibleson and G. Weiss) Weak type estimates for maximal operators on certain $H^{p}$ classes, Rend. Circ. Mat. Palermo (2) 1 (1981), 81-97.

[107] (with D. H. Phong) Some further classes of pseudo-differential and singularintegral operators arising in boundary value problems I. Composition of operators, Amer. J. Math. 104 (1982), 141-172.

[108] (with D. Geller) Singular convolution operators on the Heisenberg group, Bull. Amer. Math. Soc. (N.S.) 6 (1982), no. 1, 99-103.

[109] (with R. Fefferman) Singular integrals on product spaces, Adv. in Math. 45 (1982), 117-143.

[110] (with G. B. Folland) Hardy spaces on homogeneous groups, Mathematical Notes 28, Princeton University Press, Princeton, N.J.; Univ. Tokyo Press, Tokyo, 1982, $\mathrm{xii}+285 \mathrm{pp}$

[111] The development of square functions in the work of A. Zygmund, Bull. Amer. Math. Soc. (N.S.) 7 (1982), 359-376.

[112] (with D. M. Oberlin) Mapping properties of the Radon transform, Indiana Univ. Math. J. 31 (1982), no. 5, 641-650.

[113] An example on the Heisenberg group related to the Lewy operator, Invent. Math. 69 (1982), 209-216.

[114] (with R. Fefferman, R. Gundy, and M. Silverstein) Inequalities for ratios of functionals of harmonic functions, Proc. Nat. Acad. Sci. U.S.A. 79 (1982), 79587960 .

[115] (with D. H. Phong) Singular integrals with kernels of mixed homogeneities, Conference on harmonic analysis in honor of Antoni Zygmund, Vol. I, II (Chicago, Ill., 1981), 327-339, Wadsworth Math. Ser., Wadsworth, Belmont, CA, 1983.

[116] Some results in harmonic analysis in $\mathbf{R}^{n}$, for $n \rightarrow \infty$, Bull. Amer. Math. Soc. (N.S.) 9 (1983), 71-73.

[117] The development of square functions in the work of A. Zygmund, Conference on harmonic analysis in honor of Antoni Zygmund, Vol. I, II (Chicago, Ill., 1981), 2-30, Wadsworth Math. Ser., Wadsworth, Belmont, CA, 1983.

[118] An $H^{1}$ function with non-summable Fourier expansion, Harmonic analysis (Cortona, 1982), 193-200, Lecture Notes in Math. 992, Springer, Berlin, 1983.

[119] (with R. R. Coifman and Y. Meyer) Un nouvel éspace fonctionnel adapté à l'étude des opérateurs définis par des intégrales singulières (French), Harmonic analysis (Cortona, 1982), 1-15, Lecture Notes in Math. 992, Springer, Berlin, 1983.

[120] Boundary behavior of harmonic functions on symmetric spaces: maximal estimates for Poisson integrals, Invent. Math. 74 (1983), 63-83.

[121] (with J.-O. Strömberg) Behavior of maximal functions in $\mathbf{R}^{n}$ for large $n$, Ark. Mat. 21 (1983), 259-269.

[122] (with D. H. Phong) Singular integrals related to the Radon transform and boundary value problems, Proc. Nat. Acad. Sci. U.S.A. 80 (1983), 7697-7701.

[123] (with D. Geller) Estimates for singular convolution operators on the Heisenberg group, Math. Ann. 267 (1984), 1-15.

[124] (with A. Nagel) On certain maximal functions and approach regions, Adv. in Math. 54 (1984), 83-106.

[125] (with R. R. Coifman and Y. Meyer) Some new function spaces and their applications to harmonic analysis, J. Funct. Anal. 62 (1985), no. 2, 304-335. 
[126] Three variations on the theme of maximal functions, Recent progress in Fourier analysis (El Escorial, 1983), 229-244, North-Holland Math. Stud. 111, NorthHolland, Amsterdam, 1985.

[127] Radon singular integrals, Miniconference on linear analysis and function spaces (Canberra, 1984), 114-119, Proc. Centre Math. Anal. Austral. Nat. Univ. 9, Austral. Nat. Univ., Canberra, 1985.

[128] (with A. Nagel and S. Wainger) Balls and metrics defined byvector fields I: Basic properties, Acta Math. 155 (1985), 103-147.

[129] (with C. Sogge) Averages of functions over hypersurfaces in $\mathbb{R}^{n}$, Invent. Math. 82 (1985), 543-556.

[130] Some oscillatory integrals and their applications, Bony-Sjöstrand-Meyer seminar, 1984-1985, Exp. No. 23, 6 pp., École Polytech., Palaiseau, 1985.

[131] Oscillatory integrals in Fourier analysis, Beijing lectures in harmonic analysis (Beijing, 1984), 307-355, Ann. of Math. Stud. 112, Princeton Univ. Press, Princeton, NJ, 1986.

[132] (with D. H. Phong) Hilbert integrals, singular integrals and Radon transforms I, Acta Math. 157, (1986), 99-157.

[133] (with D. H. Phong) Hilbert integrals, singular integrals and Radon transforms II, Invent. Math. 86 (1986), 75-113.

[134] (with F. Ricci) Oscillatory singular integrals and harmonic analysis on nilpotent groups, Proc. Nat. Acad. Sci. U.S.A. 83 (1986), 1-3.

[135] (with F. Ricci) Homogeneous distributions on spaces of Hermitean matrices, J. Reine Angew. Math. 368 (1986), 142-164.

[136] (with C. D. Sogge) Averages over hypersurfaces: II, Invent. Math. 86 (1986), 233-242.

[137] (with M. Christ) A remark on singular Calderón-Zygmund theory, Proc. Amer. Math. Soc. 99 (1987), no. 1, 71-75.

[138] Problems in harmonic analysis related to curvature and oscillatory integrals, Proceedings of the International Congress of Mathematicians, Vol. 1, 2 (Berkeley, Calif., 1986), 196-221, Amer. Math. Soc., Providence, RI, 1987.

[139] (with F. Ricci) Harmonic analysis on nilpotent groups and singular integrals I: oscillatory integrals, J. Funct. Anal. 73 (1987), 179-194.

[140] (with F. Ricci) Harmonic analysis on nilpotent groups and singular integrals II: singular kernels supported on submanifolds, J. Funct. Anal. 78 (1988), 56-84.

[141] (with A. Nagel, J.-P. Rosay, and S. Wainger) Estimates for the Bergman and Szegő kernels in certain weakly pseudoconvex domains, Bull. Amer. Math. Soc. 18 (1988), 55-59.

[142] (with D.-C. Chang and A. Nagel) Estimates for the $\bar{\partial}$-Neumann problem for pseudoconvex domains in $C^{2}$ of finite type, Proc. Nat. Acad. Sci. U.S.A. 85 (1988), 8771-8774.

[143] (with A. Nagel, J.-P. Rosay, and S. Wainger) Estimates for the Bergman and Szegö kernels in $\mathbf{C}^{2}$, Ann. of Math. (2) 129 (1989), no. 1, 113-149.

[144] (with D. H. Phong) Singular Radon transforms and oscillatory integrals, Duke Math. J. 58 (1989), 347-369.

[145] (with F. Ricci) Harmonic analysis on nilpotent groups andsingular integrals III: fractional integration along manifolds, J. Funct. Anal. 86 (1989), 360-389.

[146] (with A. Nagel and F. Ricci) Fundamental solutions and harmonic analysis on nilpotent groups, Bull. Amer. Math. Soc. (N.S.) 23 (1990), no. 1, 139-144.

[147] (with A. Nagel and F. Ricci) Harmonic analysis and fundamental solutions on nilpotent Lie groups, Analysis and partial differential equations, 249-275, Lecture Notes in Pure and Appl. Math. 122, Dekker, New York, 1990.

[148] (with C. D. Sogge) Averages over hypersurfaces, smoothness of generalized Radon transforms, J. Analyse Math. 54 (1990), 165-188. 
[149] (with S. Sahi) Analysis in matrix space and Spehs representations, Invent. Math. 101 (1990), 379-393.

[150] (with S. Wainger) Discrete analogues of singular Radon transforms, Bull. Amer. Math. Soc. (N.S.) 23 (1990), 537-544.

[151] (with E. Lieb, Elliott and A. Wightman) Valentine Bargmann 1908-1989, Notices Amer. Math. Soc. 37 (1990), no. 4, 440-441.

[152] (with D. H. Phong) Radon transforms and torsion, Internat. Math. Res. Notices 1991, no. 4, 49-60.

[153] (with A. Seeger and C. Sogge) Regularity properties of Fourier integral operators, Ann. of Math. (2) 134 (1991), 231-251.

[154] (with J. Stein) Stock price distributions with stochastic volatility: an analytic approach, Rev. Financial Stud. 4 (1991), 727-752.

[155] (with F. Ricci) Multiparameter singular integrals and maximal functions, Ann. Inst. Fourier (Grenoble) 42 (1992), no. 3, 637-670.

[156] (with D.-C. Chang and A. Nagel) Estimates for the $\bar{\partial}-$ Neumann problem in pseudoconvex domains of finite type in $\mathbf{C}^{2}$, Acta Math. 169 (1992), 153-228.

[157] (with A. Calderón) Antoni Zygmund: 1900-1992, Notices Amer. Math. Soc. 39 (1992), no. 8, 848-849.

[158] (with D. H. Phong) Oscillatory integrals with polynominal phases, Invent. Math. 110 (1992), 39-62.

[159] (with D.-C. Chang and S. Krantz) Hardy spaces and elliptic boundary value problems, Contemp. Math. 137 (1992), 119-131.

[160] (with D. C. Chang and S. Krantz) $H^{p}$ theory on a smooth domain in $\mathbf{R}^{N}$ and elliptic boundary value problems, J. Funct. Anal. 114 (1993), 286-347.

[161] Harmonic analysis: real-variable methods, orthogonality, and oscillatory integrals, Princeton Mathematical Series 43, Princeton University Press, Princeton, NJ, 1993, xiv+695 pp.

[162] (with D. H. Phong) Operator versions of the van der Corput lemma and Fourier integral operators, Math. Res. Lett. 1 (1994), 27-33.

[163] (with D. H. Phong) On a stopping process for oscillatory integrals, J. Geom. Anal. 4 (1994), 105-120.

[164] (with J. McNeal) Mapping properties of the Bergman projection on convex domains of finite type, Duke Math. J. 73 (1994), 177-199.

[165] (with D. Müller) On spectral multipliers for the Heisenberg and related groups, J. Math. Pures Appl. (9) 73, (1994), 413-440.

[166] (with D. H. Phong) Models of degenerate Fourier integral operators and Radon transforms, Ann. of Math. (2) 140 (1994), 703-722.

[167] (with A. Nevo) A generalization of Birkhoff's pointwise ergodic theorem, Acta Math. 173 (1994), 135-154.

[168] (with D. Müller and F. Ricci) Marcinkiewicz multipliers and multi-parameter structure on Heisenberg (-type) groups I, Invent. Math. 119 (1995), 199-233.

[169] Oscillatory integrals related to Radon-like transforms, J. Fourier Anal. Appl., Kahane Special Issue (1995), 535-551.

[170] Spectral multipliers and multiple-parameter structures on the Heisenberg group, Journées "Équations aux Dérivées Partielles," (Saint-Jean-de-Monts, 1995), Exp. No. XVI, 15 pp., École Polytech., Palaiseau, 1995.

[171] (with D. Müller and F. Ricci) Marcinkiewicz multipliers and multi-parameter structure on Heisenberg (-type) groups II, Math. Z. 221 (1996), 267-291.

[172] (with J. McNeal) The Szegö projection on convex domains, Math. Z. 224 (1997), 519-533.

[173] (with A. Nevo) Analogs of Wiener's ergodic theorem for semisimple groups I, Ann. of Math. 145 (1997), 565-595. 
[174] (with D. H. Phong) The Newton polyhedron and oscillatory integral operators, Acta Math. 179 (1997), 105-152.

[175] (with D. H. Phong) Damped oscillatory integral operators with analytic phases, Adv. Math. 134 (1998), 146-177.

[176] Singular Integrals: The Roles of Calderón and Zygmund, Notices Amer. Math. Soc. 45 (1998), no. 9, 1130-1140.

[177] (with C. E. Kenig) Multilinear estimates and fractional integration, Math. Res. Lett. 6, (1999), 1-15.

[178] (with D.-C. Chang and G. Dafni) Hardy spaces, BMO, and boundary value problems for the Laplacian on a smooth domain in $\mathbf{R}^{n}$, Trans. Amer. Math. Soc. 351 (1999), 1605-1661.

[179] (with D. H. Phong and J. Sturm) On the growth and stability of real-analytic functions, Amer. J. Math. 121 (1999), 519-554.

[180] (with D. Müller) $L^{p}$-estimates for the wave equation on the Heisenberg group, Rev. Mat. Iberoamericana 15 (1999), no. 2, 297-334.

[181] (with S. Wainger) Discrete analogues in harmonic analysis $I: l^{2}$ estimates for singular Radon transforms, Amer. J. Math. 121 (1999), no. 6, 1291-1336.

[182] (with M. Christ, A. Nagel, and S. Wainger) Singular and maximal Radon transforms: analysis and geometry, Ann. of Math. (2) 150 (1999), no. 2, 489-577.

[183] Calderón and Zygmund's theory of singular integrals, Harmonic analysis and partial differential equations (Chicago, IL, 1996), 1-26, Chicago Lectures in Math., Univ. Chicago Press, Chicago, IL, 1999.

[184] (with G. A. Margulis and A. Nevo) Analogs of Wiener's ergodic theorems for semi-simple Lie groups II, Duke Math. J. 103 (2000), 233-259.

[185] Some geometrical concepts arising in harmonic analysis, GAFA 2000 (Tel Aviv, 1999), Geom. Funct. Anal. 2000, Special Volume, Part I, 434-453.

[186] (with S. Wainger) Discrete analogues in harmonic analysis II: fractional integration, J. Anal. Math. 80 (2000), 335-355.

[187] (with D. H. Phong and J. Sturm) Multilinear level set operators, oscillatory integral operators, and Newton polyhedra, Math. Ann. 319 (2001), no. 3, 573-596.

[188] (with A. Nagel and F. Ricci) Singular integrals with flag kernels and analysis on quadratic CR manifolds, J. Funct. Anal. 181 (2001), no. 1, 29-118.

[189] (with A. Nagel) Differentiable control metrics and scaled bump functions, J. Differential Geom. 57 (2001), no. 3, 465-492.

[190] (with A. Nagel) The $\square_{b}$-heat equation on pseudoconvex manifolds of finite type in $\mathbb{C}^{2}$, Math. Z. 238 (2001), 37-88.

[191] (with S. Wainger) Oscillatory integrals related to Carleson's theorem, Math. Res. Lett. 8 (2001), 789-800.

[192] (with A. Magyar and S. Wainger) Discrete analogues in harmonic analysis: spherical averages, Ann. of Math. (2) 155 (2002), 189-208.

[193] (with S. Wainger) Two discrete fractional integral operators revisited. Dedicated to the memory of Thomas H. Wolff, J. Anal. Math. 87 (2002), 451-479.

[194] (with R. Shakarchi) Fourier analysis. An introduction, Princeton Lectures in Analysis 1, Princeton University Press, Princeton, NJ, 2003, xvi+311 pp.

[195] (with R. Shakarchi) Complex analysis, Princeton Lectures in Analysis 2, Princeton University Press, Princeton, NJ, 2003, xviii+379 pp.

[196] (with A. Nagel) On the product theory of singular integrals, Rev. Mat. Iberoamericana 20 (2004), no. 2, 531-561.

[197] (with L. Lanzani) Szegö and Bergman projections on non-smooth planar domains, J. Geom. Anal. 14 (2004), no. 1, 63-86.

[198] (with R. Shakarchi) Real analysis. Measure theory, integration, and Hilbert spaces, Princeton Lectures in Analysis 3, Princeton University Press, Princeton, NJ, 2005, xx+402 pp. 
[199] (with L. Lanzani) A note on the div curl inequalities, Math. Res. Lett. 12 (2005), 57-61.

[200] (with A. Nagel) The $\bar{\partial}_{b}$-complex on decoupled boundaries in $\mathbb{C}^{n}$, Ann. of Math. (2) 164 (2006), 649-713.

[201] (with P. Gressman) Regularity of the Fourier transform on spaces of homogeneous distributions, J. Anal. Math. 100 (2006), 211-222.

[202] (with A. Gulisashvili) Asymptotic behavior of the distribution of the stock price in models with stochastic volatility: the Hull-White model, C. R. Math. Acad. Sci. Paris 343 (2006), 519-523.

[203] (with A. Magyar and S. Wainger) Maximal operators associated to discrete subgroups of nilpotent Lie groups, J. Anal. Math. 101 (2007), 257-312.

[204] (with A. Ionescu, A. Magyar, and S. Wainger) Discrete Radon transforms and applications to ergodic theory, Acta Math. 198 (2007), 231-298.

[205] (with C. Fefferman) Commentary on Calderón's papers on interpolation, Selected papers of Alberto P. Calderón, 567-572, Amer. Math. Soc., Providence, RI, 2008.

[206] (with C. Fefferman) Comments on several papers of A. P. Calderón, Selected papers of Alberto P. Calderón, 573-587, Amer. Math. Soc., Providence, RI, 2008.

[207] (with A. Gulisashvili) Implied volatility in the Hull-White model, Math. Finance 19 (2009), 303-327.

[208] (with A. Gulisashvili) Asymptotic behavior of distribution densities in models with stochastic volatility I, Math. Finance 20 (2010), 447-477.

[209] (with A. Gulisashvili) Asymptotic behavior of the stock price distribution density and implied volatility in stochastic volatility models, Applied Math. Optim. 61 (2010), 287-315.

[210] (with B. Street) Multi-parameter singular Radon transforms, Math. Res. Lett. 18 (2011), 257-277.

[211] (with R. Shakarchi) Functional analysis. Introduction to further topics in analysis, Princeton Lectures in Analysis 4, Princeton University Press, Princeton, NJ, 2011, xviii+423 pp.

[212] (with A. Nagel, F. Ricci, and S. Wainger) Singular integrals with flag kernels on homogeneous groups I, Rev. Mat. Iberoam. 28 (2012), no. 3, 631-722.

[213] (with B. Street) Multi-parameter singular Radon transforms III: Real analytic surfaces, Adv. Math. 229 (2012), 2210-2238.

[214] (with L. Lanzani) The Bergman projection in $L^{p}$ for domains with minimal smoothness, Illinois J. Math. 56 (2012), no. 1, 127-154.

[215] (with B. Street) Multi-parameter singular Radon transforms II: The $L^{p}$ theory, Adv. Math. 248 (2013), 736-783.

[216] (with L. Lanzani) Cauchy-type integrals in several complex variables, Bull. Math. Sci. 3 (2013), no. 2, 241-285.

[217] (with P.-L. Yung) Pseudodifferential operators of mixed type adapted to distributions of k-planes, Math. Res. Lett. 20 (2013), no. 6, 1183-1208.

[218] Three projection operators in complex analysis, Colloquium De Giorgi 2010-2012, 49-59, Colloquia 4, Ed. Norm., Pisa, 2013.

[219] (with L. Lanzani) The Cauchy integral in $\mathbb{C}^{n}$ for domains with minimal smoothness, Adv. Math. 264 (2014), 776-830.

[220] (with L. Lanzani) Hardy spaces of holomorphic functions for domains in $\mathbb{C}^{n}$ with minimal smoothness, Harmonic analysis, partial differential equations, complex analysis, Banach spaces, and operator theory. Vol. 1, 179-199, Assoc. Women Math. Ser. 4, Springer, 2016.

[221] (with L. Lanzani) The Cauchy-Szegő projection for domains in $\mathbb{C}^{n}$ with minimal smoothness, Duke Math. J. 166 (2017), 125-176. 
[222] (with M. Mirek and B. Trojan) $\ell^{p}\left(\mathbb{Z}^{d}\right)$-estimates for discrete operators of Radon type: variational estimates, Invent. Math. 209 (2017), 665-748.

[223] (with L. Lanzani) The role of an integration identity in the analysis of the CauchyLeray transform, Sci. China Math. 60 (2017), no. 11, 1923-1936.

[224] (with A. Nagel, F. Ricci, and S. Wainger) Algebras of singular integral operators with kernels controlled by multiple norms, Mem. Amer. Math. Soc. 256 (2018), no. 1230 , vii+141 pp.

[225] (with J. Bourgain, M. Mirek, and B. Wróbel) On dimension-free variational inequalities for averaging operators in $\mathbb{R}^{d}$, Geom. Funct. Anal. 28 (2018), no. 1, 58-99.

[226] (with M. Mirek and B. Trojan) $\ell^{p}\left(\mathbb{Z}^{d}\right)$-estimates for discrete operators of Radon type: maximal functions and vector-valued estimates, J. Funct. Anal. 277 (2019), 2471-2521.

[227] (with J. Bourgain, M. Mirek, and B. Wróbel) Dimension-free estimates for discrete Hardy-Littlewood averaging operators over the cubes in $\mathbb{Z}^{d}$, Amer. J. Math. 141 (2019), no. 4, 857-905.

[228] (with L. Lanzani) The Cauchy-Leray integral: counter-examples to the $L^{p}$-theory, arXiv:1701.03812 (2017).

[229] (with M. Mirek and P. Zorin-Kranich), Jump inequalities via real interpolation, Math. Ann. (to appear), arXiv:1808.04592 (2018).

[230] (with M. Mirek and P. Zorin-Kranich), A bootstrapping approach to jump inequalities and their applications, Anal. PDE (to appear), arXiv:1808.09048 (2018).

[231] (with M. Mirek and P. Zorin-Kranich), Jump inequalities for translation-invariant operators of Radon type on $\mathbb{Z}^{d}$, arXiv:1809.03803 (2018).

[232] (with J. Bourgain, M. Mirek, and B. Wróbel), On the Hardy-Littlewood maximal functions in high dimensions: continuous and discrete perspective, arXiv:1812. 00153 (2018).

[233] (with J. Bourgain, M. Mirek, and B. Wróbel), On discrete Hardy-Littlewood maximal functions over the balls in $\mathbb{Z}^{d}$ : dimension-free estimates, arXiv:1812.00154 (2018).

[234] (with L. Lanzani) On regularity and irregularity of certain holomorphic singular integral operators, arXiv:1901.03402 (2019).

\section{REFERENCES}

[Al] J. M. Aldaz, The weak type $(1,1)$ bounds for the maximal function associated to cubes grow to infinity with the dimension, Ann. of Math. (2) 173 (2011), no. 2, 1013-1023, DOI 10.4007/annals.2011.173.2.10. MR2776368

[Ba] D. E. Barrett, Behavior of the Bergman projection on the Diederich-Forncess worm, Acta Math. 168 (1992), no. 1-2, 1-10, DOI 10.1007/BF02392975. MR1149863

[BL] D. E. Barrett and L. Lanzani, The spectrum of the Leray transform for convex Reinhardt domains in $\mathbb{C}^{2}$, J. Funct. Anal. 257 (2009), no. 9, 2780-2819, DOI 10.1016/j.jfa.2009.04.011. MR2559717

[BFG] M. Beals, C. Fefferman, and R. Grossman, Strictly pseudoconvex domains in $\mathbf{C}^{n}$, Bull. Amer. Math. Soc. (N.S.) 8 (1983), no. 2, 125-322, DOI 10.1090/S0273-0979-1983-150875. MR684898

[BL] V. Bergelson and A. Leibman, A nilpotent Roth theorem, Invent. Math. 147 (2002), no. 2, 429-470, DOI 10.1007/s002220100179. MR1881925

[Bo1] J. Bourgain, Averages in the plane over convex curves and maximal operators, J. Analyse Math. 47 (1986), 69-85, DOI 10.1007/BF02792533. MR874045

[Bo2] J. Bourgain, On high-dimensional maximal functions associated to convex bodies, Amer. J. Math. 108 (1986), no. 6, 1467-1476, DOI 10.2307/2374532. MR868898

[Bo3] J. Bourgain, On the $L^{p}$-bounds for maximal functions associated to convex bodies in $\mathbf{R}^{n}$, Israel J. Math. 54 (1986), no. 3, 257-265, DOI 10.1007/BF02764955. MR853451 
[Bo4] J. Bourgain, On the maximal ergodic theorem for certain subsets of the integers, Israel J. Math. 61 (1988), no. 1, 39-72, DOI 10.1007/BF02776301. MR937581

[Bo5] J. Bourgain, On the pointwise ergodic theorem on $L^{p}$ for arithmetic sets, Israel J. Math. 61 (1988), no. 1, 73-84, DOI 10.1007/BF02776302. MR.937582

[Bo6] J. Bourgain, A remark on the maximal function associated to an analytic vector field, Analysis at Urbana, Vol. I (Urbana, IL, 1986), London Math. Soc. Lecture Note Ser., vol. 137, Cambridge Univ. Press, Cambridge, 1989, pp. 111-132. MR1009171

[Bo7] J. Bourgain, Pointwise ergodic theorems for arithmetic sets, Inst. Hautes Etudes Sci. Publ. Math. 69 (1989), 5-45. With an appendix by the author, Harry Furstenberg, Yitzhak Katznelson and Donald S. Ornstein. MR 1019960

[Bo8] J. Bourgain, Some new estimates on oscillatory integrals, Essays on Fourier analysis in honor of Elias M. Stein (Princeton, NJ, 1991), Princeton Math. Ser., vol. 42, Princeton Univ. Press, Princeton, NJ, 1995, pp. 83-112. MR1315543

[Bo9] J. Bourgain, On the Hardy-Littlewood maximal function for the cube, Israel J. Math. 203 (2014), no. 1, 275-293, DOI 10.1007/s11856-014-1059-2. MR3273441

[BD] J. Bourgain and C. Demeter, The proof of the $l^{2}$ decoupling conjecture, Ann. of Math. (2) 182 (2015), no. 1, 351-389, DOI 10.4007/annals.2015.182.1.9. MR3374964

[BDG] J. Bourgain, C. Demeter, and L. Guth, Proof of the main conjecture in Vinogradov's mean value theorem for degrees higher than three, Ann. of Math. (2) 184 (2016), no. 2, 633-682, DOI 10.4007/annals.2016.184.2.7. MR3548534

[BGS] D. L. Burkholder, R. F. Gundy, and M. L. Silverstein, A maximal function characterization of the class $H^{p}$, Trans. Amer. Math. Soc. 157 (1971), 137-153, DOI 10.2307/1995838. MR 274767

[BDIM] S. Buschenhenke, S. Dendrinos, I. Ikromov, and D. Müller, Estimates for maximal functions associated to hypersurfaces in $\mathbb{R}^{3}$ with height $h<2$, Part I, arXiv:1704.06520 (2017).

[Ca1] A.-P. Calderón, Commutators of singular integral operators, Proc. Nat. Acad. Sci. U.S.A. 53 (1965), 1092-1099, DOI 10.1073/pnas.53.5.1092. MR.177312

[Ca2] A.-P. Calderón, Cauchy integrals on Lipschitz curves and related operators, Proc. Nat. Acad. Sci. U.S.A. 74 (1977), no. 4, 1324-1327, DOI 10.1073/pnas.74.4.1324. MR466568

[CZ] A.-P. Calderón and A. Zygmund, On higher gradients of harmonic functions, Studia Math. 24 (1964), 211-226, DOI 10.4064/sm-24-2-211-226. MR.167631

[Cb] A. Carbery, An almost-orthogonality principle with applications to maximal functions associated to convex bodies, Bull. Amer. Math. Soc. (N.S.) 14 (1986), no. 2, 269-273, DOI 10.1090/S0273-0979-1986-15436-4. MR828824

[CCW] A. Carbery, M. Christ, and J. Wright, Multidimensional van der Corput and sublevel set estimates, J. Amer. Math. Soc. 12 (1999), no. 4, 981-1015, DOI 10.1090/S0894-0347-9900309-4. MR,1683156

[Cr] L. Carleson, On convergence and growth of partial sums of Fourier series, Acta Math. 116 (1966), 135-157, DOI 10.1007/BF02392815. MR199631

[Ch1] M. Christ, Hilbert transforms along curves. I. Nilpotent groups, Ann. of Math. (2) 122 (1985), no. 3, 575-596, DOI 10.2307/1971330. MR819558

[Ch2] M. Christ, Pointwise estimates for the relative fundamental solution of $\bar{\partial}_{b}$, Proc. Amer. Math. Soc. 104 (1988), no. 3, 787-792, DOI 10.2307/2046793. MR929407

[Ch3] M. Christ, Regularity properties of the $\bar{\partial}_{b}$ equation on weakly pseudoconvex CR manifolds of dimension 3, J. Amer. Math. Soc. 1 (1988), no. 3, 587-646, DOI 10.2307/1990950. MR928903

[Ch4] M. Christ, On the $\bar{\partial}_{b}$ equation and Szegö projection on CR manifolds, Harmonic analysis and partial differential equations (El Escorial, 1987), Lecture Notes in Math., vol. 1384, Springer, Berlin, 1989, pp. 146-158, DOI 10.1007/BFb0086799. MR.1013821

[Ch5] M. Christ, A T (b) theorem with remarks on analytic capacity and the Cauchy integral, Colloq. Math. 60/61 (1990), no. 2, 601-628, DOI 10.4064/cm-60-61-2-601-628. MR:1096400

[Ch6] M. Christ, On the $\bar{\partial}_{b}$ equation for three-dimensional CR manifolds, Several complex variables and complex geometry, Part 3 (Santa Cruz, CA, 1989), Proc. Sympos. Pure Math., vol. 52, Amer. Math. Soc., Providence, RI, 1991, pp. 63-82. MR1128584

[Ch7] M. Christ, The strong maximal function on a nilpotent group, Trans. Amer. Math. Soc. 331 (1992), no. 1, 1-13, DOI 10.2307/2153994. MR.1104197 
[CMM] R. R. Coifman, A. McIntosh, and Y. Meyer, L'intégrale de Cauchy définit un opérateur borné sur $L^{2}$ pour les courbes lipschitziennes (French), Ann. of Math. (2) 116 (1982), no. 2, 361-387, DOI 10.2307/2007065. MR672839

[CM] R. R. Coifman and Y. Meyer, Au delà des opérateurs pseudo-différentiels (French), Astérisque, vol. 57, Société Mathématique de France, Paris, 1978. With an English summary. MR518170

[CGP] T. C. Collins, A. Greenleaf, and M. Pramanik, A multi-dimensional resolution of singularities with applications to analysis, Amer. J. Math. 135 (2013), no. 5, 1179-1252, DOI 10.1353/ajm.2013.0042. MR3117305

[Co] M. Cotlar, A unified theory of Hilbert transforms and ergodic theorems (English, with Spanish summary), Rev. Mat. Cuyana 1 (1955), 105-167 (1956). MR0084632

[Cw] M. Cowling, The Kunze-Stein phenomenon, Ann. Math. (2) 107 (1978), no. 2, 209-234, DOI 10.2307/1971142. MR0507240

[Da] G. David, Opérateurs intégraux singuliers sur certaines courbes du plan complexe (French), Ann. Sci. École Norm. Sup. (4) 17 (1984), no. 1, 157-189. MR744071

[DGM] L. Deleaval, O. Guédon, and B. Maurey, Dimension free bounds for the Hardy-Littlewood maximal operator associated to convex sets, arXiv:1602.02015 (2016).

[EM] L. Ehrenpreis and F. I. Mautner, Uniformly bounded representations of groups, Proc. Nat. Acad. Sci. U.S.A. 41 (1955), 231-233, DOI 10.1073/pnas.41.4.231. MR71434

[Fe1] C. Fefferman, Inequalities for strongly singular convolution operators, Acta Math. 124 (1970), 9-36, DOI 10.1007/BF02394567. MR257819

[Fe2] C. Fefferman, The multiplier problem for the ball, Ann. of Math. (2) 94 (1971), 330-336, DOI 10.2307/1970864. MR296602

[Fe3] C. Fefferman, A note on spherical summation multipliers, Israel J. Math. 15 (1973), 44-52, DOI 10.1007/BF02771772. MR320624

[Fe4] C. Fefferman, Pointwise convergence of Fourier series, Ann. of Math. (2) 98 (1973), 551-571, DOI 10.2307/1970917. MR340926

[Fe5] C. Fefferman, Parabolic invariant theory in complex analysis, Adv. in Math. 31 (1979), no. 2, 131-262, DOI 10.1016/0001-8708(79)90025-2. MR.526424

[Fe6] C. Fefferman, Selected theorems by Eli Stein, Essays on Fourier analysis in honor of Elias M. Stein (Princeton, NJ, 1991), Princeton Math. Ser., vol. 42, Princeton Univ. Press, Princeton, NJ, 1995, pp. 1-35. MR1315540

[FK1] C. L. Fefferman and J. J. Kohn, Estimates of kernels on three-dimensional CR manifolds, Rev. Mat. Iberoamericana 4 (1988), no. 3-4, 355-405, DOI 10.4171/RMI/78. MR. 1048582

[FK2] C. L. Fefferman and J. J. Kohn, Hölder estimates on domains of complex dimension two and on three-dimensional CR manifolds, Adv. in Math. 69 (1988), no. 2, 223-303, DOI 10.1016/0001-8708(88)90002-3. MR946264

[FP] C. Fefferman and D. H. Phong, Subelliptic eigenvalue problems, Conference on harmonic analysis in honor of Antoni Zygmund, Vol. I, II (Chicago, Ill., 1981), Wadsworth Math. Ser., Wadsworth, Belmont, CA, 1983, pp. 590-606. MR730094

[FS] C. L. Fefferman and A. Sánchez-Calle, Fundamental solutions for second order subelliptic operators, Ann. of Math. (2) 124 (1986), no. 2, 247-272, DOI 10.2307/1971278. MR855295

[GN] I. M. Gelfand and M. A. Neumark, Unitäre Darstellungen der klassischen Gruppen (German), Akademie-Verlag, Berlin, 1957. MR0085262

[GU] V. Guillemin and G. Uhlmann, Oscillatory integrals with singular symbols, Duke Math. J. 48 (1981), no. 1, 251-267. MR610185

[Gr] M. Greenblatt, Sharp $L^{2}$ estimates for one-dimensional oscillatory integral operators with $C^{\infty}$ phase, Amer. J. Math. 127 (2005), no. 3, 659-695. MR2141648

[GPT] A. Greenleaf, M. Pramanik, and W. Tang, Oscillatory integral operators with homogeneous polynomial phases in several variables, J. Funct. Anal. 244 (2007), no. 2, 444-487, DOI 10.1016/j.jfa.2006.11.005. MR2297031

[GS1] A. Greenleaf and A. Seeger, Oscillatory and Fourier integral operators with degenerate canonical relations, Proceedings of the 6th International Conference on Harmonic Analysis and Partial Differential Equations (El Escorial, 2000), Publ. Mat. Vol. Extra (2002), 93-141, DOI 10.5565/PUBLMAT_Esco02_05. MR 1964817 
[GS2] A. Greenleaf and A. Seeger, Oscillatory integral operators with low-order degeneracies, Duke Math. J. 112 (2002), no. 3, 397-420, DOI 10.1215/S0012-9074-02-11231-9. MR 1896469

[GrU] A. Greenleaf and G. Uhlmann, Estimates for singular Radon transforms and pseudodifferential operators with singular symbols, J. Funct. Anal. 89 (1990), no. 1, 202-232, DOI 10.1016/0022-1236(90)90011-9. MR1040963

[Gs] P. T. Gressman, Uniform estimates for cubic oscillatory integrals, Indiana Univ. Math. J. 57 (2008), no. 7, 3419-3442, DOI 10.1512/iumj.2008.57.3403. MR2492238

[GX] P. T. Gressman and L. Xiao, Maximal decay inequalities for trilinear oscillatory integrals of convolution type, J. Funct. Anal. 271 (2016), no. 12, 3695-3726, DOI 10.1016/j.jfa.2016.09.003. MR3558254

[GRY] S. Guo, J. Roos, and P.-L. Yung, Sharp variation-norm estimates for oscillatory integrals related to Carleson's theorem, arXiv:1710.10988 (2017).

[GPRY] S. Guo, L. B. Pierce, J. Roos, and P.-L. Yung, Polynomial Carleson operators along monomial curves in the plane, J. Geom. Anal. 27 (2017), no. 4, 2977-3012, DOI 10.1007/s12220-017-9790-7. MR3708001

[He] G. M. Henkin, Integral representation of functions which are holomorphic in strictly pseudoconvex regions, and some applications (Russian), Mat. Sb. (N.S.) 78 (120) (1969), 611-632. MR0249660

[Hr] C. S. Herz, On the mean inversion of Fourier and Hankel transforms, Proc. Nat. Acad. Sci. U.S.A. 40 (1954), 996-999, DOI 10.1073/pnas.40.10.996. MR63477

[Hi1] I. I. Hirschman Jr., On multiplier transformations, Duke Math. J. 26 (1959), 221-242. MR104973

[Hi2] I. I. Hirschman Jr., Multiplier transformations. II, Duke Math. J. 28 (1961), 45-56. MR 124693

[Ho] L. Hörmander, Notions of convexity, Progress in Mathematics, vol. 127, Birkhäuser Boston, Inc., Boston, MA, 1994. MR1301332

[Hg] K. Hughes, The discrete spherical averages over a family of sparse sequences, J. Anal. Math. 138 (2019), no. 1, 1-21, DOI 10.1007/s11854-019-0020-z. MR.3996030

[Hu] R. A. Hunt, On the convergence of Fourier series, Orthogonal Expansions and their Continuous Analogues (Proc. Conf., Edwardsville, Ill., 1967), Southern Illinois Univ. Press, Carbondale, Ill., 1968, pp. 235-255. MR0238019

[IKM] I. A. Ikromov, M. Kempe, and D. Müller, Estimates for maximal functions associated with hypersurfaces in $\mathbb{R}^{3}$ and related problems of harmonic analysis, Acta Math. 204 (2010), no. 2, 151-271, DOI 10.1007/s11511-010-0047-6. MR2653054

[Io1] A. D. Ionescu, An endpoint estimate for the Kunze-Stein phenomenon and related maximal operators, Ann. of Math. (2) 152 (2000), no. 1, 259-275, DOI 10.2307/2661383. MR 1792296

[Io2] A. D. Ionescu, An endpoint estimate for the discrete spherical maximal function, Proc. Amer. Math. Soc. 132 (2004), no. 5, 1411-1417, DOI 10.1090/S0002-9939-03-07207-1. MR2053347

[Io3] A. D. Ionescu, Rearrangement inequalities on semisimple Lie groups, Math. Ann. 332 (2005), no. 4, 739-758, DOI 10.1007/s00208-005-0650-6. MR2179774

[IMW] A. D. Ionescu, A. Magyar, and S. Wainger, Averages along polynomial sequences in discrete nilpotent Lie groups: singular Radon transforms, Advances in analysis: the legacy of Elias M. Stein, Princeton Math. Ser., vol. 50, Princeton Univ. Press, Princeton, NJ, 2014, pp. 146-188. MR3329850

[IW] A. D. Ionescu and S. Wainger, $L^{p}$ boundedness of discrete singular Radon transforms, J. Amer. Math. Soc. 19 (2006), no. 2, 357-383, DOI 10.1090/S0894-0347-05-00508-4. $\operatorname{MR} 2188130$

[JMZ] B. Jessen, J. Marcinkiewicz, and A. Zygmund, Note on the differentiability of multiple integrals, Fund. Math. 25 (1935), 217-234.

[JSW] R. L. Jones, A. Seeger, and J. Wright, Strong variational and jump inequalities in harmonic analysis, Trans. Amer. Math. Soc. 360 (2008), no. 12, 6711-6742, DOI 10.1090/S0002-9947-08-04538-8. MR2434308

[Ka] V. N. Karpushkin, A theorem concerning uniform estimates of oscillatory integrals when the phase is a function of two variables, J. Soviet Math. 35 (1986) 2809-2826. 
[KLM] R. Kesler, M. T. Lacey, and D. Mena, Sparse bounds for the discrete spherical maximal functions, Pure Appl. Anal. 2 (2020), no. 1, 75-92, DOI 10.2140/paa.2020.2.75. MR 4041278

[Ko] K. D. Koenig, On maximal Sobolev and Hölder estimates for the tangential CauchyRiemann operator and boundary Laplacian, Amer. J. Math. 124 (2002), no. 1, 129-197. MR.1879002

[Kl] A. Kolmogorov, Une série de Fourier-Lebesgue divergente presque partout, Fund. Math. 4 (1923), 324-328.

[Kö] T. W. Körner, Fourier analysis, Cambridge University Press, Cambridge, 1988. MR924154

[LT] M. Lacey and C. Thiele, A proof of boundedness of the Carleson operator, Math. Res. Lett. 7 (2000), no. 4, 361-370, DOI 10.4310/MRL.2000.v7.n4.a1. MR.1783613

[Li1] V. Lie, The (weak- $L^{2}$ ) boundedness of the quadratic Carleson operator, Geom. Funct. Anal. 19 (2009), no. 2, 457-497, DOI 10.1007/s00039-009-0010-x. MR2545246

[Li2] V. Lie, A note on the polynomial Carleson operator in higher dimensions, arXiv:1712.03092 (2017).

[Lu] N. N. Luzin, Integral i trigonometričeskǐ ryad (Russian), Gosudarstv. Izdat. Tehn.-Teor. Lit., Moscow-Leningrad, 1951. Editing and commentary by N. K. Bari and D. E. Men'šov. MR0048364

[Ma] M. Machedon, Estimates for the parametrix of the Kohn Laplacian on certain domains, Invent. Math. 91 (1988), no. 2, 339-364, DOI 10.1007/BF01389371. MR922804

[Mg] A. Magyar, $L^{p}$-bounds for spherical maximal operators on $\mathbf{Z}^{n}$, Rev. Mat. Iberoamericana 13 (1997), no. 2, 307-317, DOI 10.4171/RMI/222. MR1617657

[Mu G. Mauceri, Zonal multipliers on the Heisenberg group, Pacific J. Math. 95 (1981), no. 1, 143-159. MR631666

[Mc] J. D. McNeal, Boundary behavior of the Bergman kernel function in $\mathbf{C}^{2}$, Duke Math. J. 58 (1989), no. 2, 499-512, DOI 10.1215/S0012-7094-89-05822-5. MR.1016431

[Mi] M. Mirek, Square function estimates for discrete Radon transforms, Anal. PDE 11 (2018), no. 3, 583-608, DOI 10.2140/apde.2018.11.583. MR3738256

[Mü] D. Müller, A geometric bound for maximal functions associated to convex bodies, Pacific J. Math. 142 (1990), no. 2, 297-312. MR1042048

[NRW1] A. Nagel, N. Rivière, and S. Wainger, On Hilbert transforms along curves, Bull. Amer. Math. Soc. 80 (1974), 106-108, DOI 10.1090/S0002-9904-1974-13374-4. MR450899

[NRW2] A. Nagel, N. Riviere, and S. Wainger, A maximal function associated to the curve $\left(t, t^{2}\right)$, Proc. Nat. Acad. Sci. U.S.A. 73 (1976), no. 5, 1416-1417, DOI 10.1073/pnas.73.5.1416. MR399389

[NRW3] A. Nagel, N. M. Rivière, and S. Wainger, On Hilbert transforms along curves. II, Amer. J. Math. 98 (1976), no. 2, 395-403, DOI 10.2307/2373893. MR450900

[NW] A. Nagel and S. Wainger, $L^{2}$ boundedness of Hilbert transforms along surfaces and convolution operators homogeneous with respect to a multiple parameter group, Amer. J. Math. 99 (1977), no. 4, 761-785, DOI 10.2307/2373864. MR450901

[NTV] F. Nazarov, S. Treil, and A. Volberg, The Tb-theorem on non-homogeneous spaces, Acta Math. 190 (2003), no. 2, 151-239, DOI 10.1007/BF02392690. MR1998349

[Ob] D. M. Oberlin, Two discrete fractional integrals, Math. Res. Lett. 8 (2001), no. 1-2, 1-6, DOI 10.4310/MRL.2001.v8.n1.a1. MR.1825254

[Ph] D. H. Phong, On integral representations for the Neumann operator, Proc. Nat. Acad. Sci. U.S.A. 76 (1979), no. 4, 1554-1558, DOI 10.1073/pnas.76.4.1554. MR526179

[Pi1] L. B. Pierce, On discrete fractional integral operators and mean values of Weyl sums, Bull. Lond. Math. Soc. 43 (2011), no. 3, 597-612, DOI 10.1112/blms/bdq127. $\operatorname{MR} 2820148$

[Pi2] L. B. Pierce, Discrete fractional Radon transforms and quadratic forms, Duke Math. J. 161 (2012), no. 1, 69-106, DOI 10.1215/00127094-1507288. MR2872554

[PY] L. B. Pierce and P.-L. Yung, A polynomial Carleson operator along the paraboloid, Rev. Mat. Iberoam. 35 (2019), no. 2, 339-422, DOI 10.4171/rmi/1057. MR.3945729

[Ra] R. M. Range, Holomorphic functions and integral representations in several complex variables, Graduate Texts in Mathematics, vol. 108, Springer-Verlag, New York, 1986. MR847923 
[Ry] V.S. Rychkov, Sharp $L^{2}$ bounds for oscillatory integral operators with $C^{\infty}$ phases, Math. Z. 236 (2001), no. 3, 461-489, DOI 10.1007/PL00004838. MR1821301

[Se] A. Seeger, Radon transforms and finite type conditions, J. Amer. Math. Soc. 11 (1998), no. 4, 869-897, DOI 10.1090/S0894-0347-98-00280-X. MR.1623430

[Sj] P. Sjölin, Convergence almost everywhere of certain singular integrals and multiple Fourier series, Ark. Mat. 9 (1971), 65-90, DOI 10.1007/BF02383638. MR336222

[So1] C. D. Sogge, Concerning the $L^{p}$ norm of spectral clusters for second-order elliptic operators on compact manifolds, J. Funct. Anal. 77 (1988), no. 1, 123-138, DOI 10.1016/00221236(88)90081-X. MR930395

[So2] C. D. Sogge, Propagation of singularities and maximal functions in the plane, Invent. Math. 104 (1991), no. 2, 349-376, DOI 10.1007/BF01245080. MR1098614

[St1] B. Street, Multi-parameter Carnot-Carathéodory balls and the theorem of Frobenius, Rev. Mat. Iberoam. 27 (2011), no. 2, 645-732, DOI 10.4171/RMI/650. MR2848534

[St2] B. Street, Multi-parameter singular Radon transforms I: The L ${ }^{2}$ theory, J. Anal. Math. 116 (2012), 83-162, DOI 10.1007/s11854-012-0004-8. MR2892618

[Sr] R. S. Strichartz, Restrictions of Fourier transforms to quadratic surfaces and decay of solutions of wave equations, Duke Math. J. 44 (1977), no. 3, 705-714. MR512086

[Sb] J.-O. Strömberg, Weak type $L^{1}$ estimates for maximal functions on noncompact symmetric spaces, Ann. of Math. (2) 114 (1981), no. 1, 115-126, DOI 10.2307/1971380. MR625348

[Ta1] T. Tao, The Bochner-Riesz conjecture implies the restriction conjecture, Duke Math. J. 96 (1999), no. 2, 363-375, DOI 10.1215/S0012-7094-99-09610-2. MR1666558

[Ta2] T. Tao, Some recent progress on the restriction conjecture, Fourier analysis and convexity, Appl. Numer. Harmon. Anal., Birkhäuser Boston, Boston, MA, 2004, pp. 217-243, DOI 10.1198/106186003321335099. MR2087245

[TW] T. Tao and J. Wright, $L^{p}$ improving bounds for averages along curves, J. Amer. Math. Soc. 16 (2003), no. 3, 605-638, DOI 10.1090/S0894-0347-03-00420-X. MR1969206

[To] X. Tolsa, Analytic capacity, the Cauchy transform, and non-homogeneous CalderónZygmund theory, Progress in Mathematics, vol. 307, Birkhäuser/Springer, Cham, 2014. MR.3154530

[Tm1] P. A. Tomas, A restriction theorem for the Fourier transform, Bull. Amer. Math. Soc. 81 (1975), 477-478, DOI 10.1090/S0002-9904-1975-13790-6. MR358216

[Tm2] P. A. Tomas, Restriction theorems for the Fourier transform, Harmonic analysis in Euclidean spaces (Proc. Sympos. Pure Math., Williams Coll., Williamstown, Mass., 1978), Proc. Sympos. Pure Math., XXXV, Part, Amer. Math. Soc., Providence, R.I., 1979, pp. 111-114. MR.545245

[Va] A. N. Varčenko, Newton polyhedra and estimates of oscillatory integrals (Russian), Funkcional. Anal. i Priložen. 10 (1976), no. 3, 13-38. MR0422257

[Zo] P. Zorin-Kranich, Maximal polynomial modulations of singular integrals, arXiv:1711. 03524 (2017).

[Zy1] A. Zygmund, Trigonometric series: Vols. I, II, Second edition, reprinted with corrections and some additions, Cambridge University Press, London-New York, 1968. MR0236587

[Zy2] A. Zygmund, On Fourier coefficients and transforms of functions of two variables, Studia Math. 50 (1974), 189-201, DOI 10.4064/sm-50-2-189-201. MR387950 
Princeton University

Email address: cf@math.princeton.edu

$U R L:$ https://www.math.princeton.edu/people/charles-fefferman

Princeton University

Email address: aionescu@math.princeton.edu

$U R L:$ https://web.math.princeton.edu/ aionescu/

University of California, Los Angeles

Email address: tao@math.ucla.edu

URL: https://www.math.ucla.edu/ tao/

UNIVERSITY OF WISCONSIN-MADISON

Email address: wainger@math.wisc.edu

URL: https://www.math.wisc.edu/ wainger/

$U R L:$ https ://thecollege.syr.edu/people/faculty/lanzani-loredana/

$U R L:$ http://math.uga.edu/ magyar/

$U R L:$ https://www .math.rutgers.edu/component/comprofiler/userprofile/1050 -mariuszmirek

$U R L:$ https ://www.math.wisc.edu/ nagel/

URL: http://www.math.columbia.edu/ phong/

$U R L:$ https://services.math.duke.edu/ pierce/

$U R L:$ http://homepage.sns.it/fricci/

$U R L:$ https://mathematics.jhu.edu/directory/christopher-sogge/

$U R L:$ https://www.math.wisc.edu/ street/ 